Supporting Information

\title{
Polyketide Production of Pestaloficiols and Macrodiolide Ficiolides Revealed by Manipulations of Epigenetic Regulators in an Endophytic Fungus
}

Guangwei Wu, ${ }^{\dagger}$ Haichuan Zhou, ${ }^{\dagger}$ Peng Zhang, ${ }^{\dagger}$ Xiuna Wang, ${ }^{\dagger}$ Wei $\mathrm{Li}^{+}{ }^{\dagger}$ Weiwei Zhang, ${ }^{\dagger}$ Xingzhong Liu, ${ }^{\dagger}$ Hong-Wei Liu,${ }^{\dagger}$ Nancy P. Keller, ${ }^{\ddagger}$ Zhiqiang An,${ }^{\S}$ and Wen-Bing Yin ${ }^{*}, \dagger$

${ }^{*}$ Corresponding author:

Wen-Bing Yin, E-mail: yinwb@im.ac.cn. Tel: 86-10-64806170

${ }^{\dagger}$ State Key Laboratory of Mycology, Institute of Microbiology, Chinese Academy of Sciences, Beijing, 100101, China

${ }^{\ddagger}$ Department of Medical Microbiology and Immunology, University of Wisconsin-Madison, WI, 53706, USA

§ Texas Therapeutics Institute, the Brown Foundation Institute of Molecular Medicine, University of Texas Health Science Center at Houston, Houston, Texas, 77030, USA. 


\section{Table of contents}

\section{Supplementary methods}

\section{Supplementary Tables}

Table S1. Summary of polyketide synthase (PKS) in Pestalotiopsis fici

Table S2. Summary of non-ribosomal peptide synthase (NRPS) and NRPS-like in Pestalotiopsis fici

Table S3. Hybrid PKS-NRPS and terpene synthases encoded in Pestalotiopsis fici

Table S4. Fungal strains and plasmids used in this study

Table S5. PCR primer sets used in this study

Table S6. ${ }^{1} \mathrm{H}(500 \mathrm{MHz})$ and ${ }^{13} \mathrm{C}(125 \mathrm{MHz})$ NMR spectroscopic data for 1 in Acetone- $d_{6}$.

Table S7. ${ }^{1} \mathrm{H}(500 \mathrm{MHz})$ and ${ }^{13} \mathrm{C}(125 \mathrm{MHz})$ NMR spectroscopic data for 2 in Acetone- $d_{6}$

Table S8. ${ }^{1} \mathrm{H}(500 \mathrm{MHz})$ and ${ }^{13} \mathrm{C}(125 \mathrm{MHz})$ NMR spectroscopic data for 3 in Acetone- $d_{6}$.

Table S9. ${ }^{1} \mathrm{H}(500 \mathrm{MHz})$ and ${ }^{13} \mathrm{C}(125 \mathrm{MHz})$ NMR spectroscopic data for 4 in DMSO- $d_{6}$.

Table S10. ${ }^{1} \mathrm{H}(500 \mathrm{MHz})$ and ${ }^{13} \mathrm{C}(125 \mathrm{MHz})$ NMR spectroscopic data for 6 in methanol- $d_{4}$.

Table S11. ${ }^{1} \mathrm{H}(500 \mathrm{MHz})$ and ${ }^{13} \mathrm{C}(125 \mathrm{MHz})$ NMR spectroscopic data for $\mathbf{7}$ and $\mathbf{8}$ in methanol- $d_{4}$.

Table S12. ${ }^{1} \mathrm{H}(500 \mathrm{MHz})$ and ${ }^{13} \mathrm{C}(125 \mathrm{MHz})$ NMR spectroscopic data for 9 and 10 in methanol- $d_{4}$.

Table S13. ${ }^{1} \mathrm{H}(500 \mathrm{MHz})$ and ${ }^{13} \mathrm{C}(125 \mathrm{MHz})$ NMR spectroscopic data for 11 in methanol- $d_{4}$.

Table S14. ${ }^{1} \mathrm{H}(500 \mathrm{MHz})$ and ${ }^{13} \mathrm{C}(125 \mathrm{MHz}) \mathrm{NMR}$ spectroscopic data for 12 in methanol- $d_{4}$.

Table S15. ${ }^{1} \mathrm{H}(500 \mathrm{MHz})$ and ${ }^{13} \mathrm{C}(125 \mathrm{MHz})$ NMR spectroscopic data for 13 and 14 in methanol- $d_{4}$.

Table S16. ${ }^{1} \mathrm{H}(500 \mathrm{MHz})$ and ${ }^{13} \mathrm{C}(125 \mathrm{MHz})$ NMR spectroscopic data for 15 in methanol- $d_{4}$.

Table S17. ${ }^{1} \mathrm{H}(500 \mathrm{MHz})$ and ${ }^{13} \mathrm{C}(125 \mathrm{MHz})$ NMR spectroscopic data for 16 in methanol- $d_{4}$.

Table S18. ${ }^{1} \mathrm{H}$ and ${ }^{13} \mathrm{C}$ NMR spectroscopic data for 5 and 21.

Table S19. ${ }^{1} \mathrm{H}(500 \mathrm{MHz})$ and ${ }^{13} \mathrm{C}(125 \mathrm{MHz})$ NMR spectroscopic data for $\mathbf{H 3}$ in methanol- $d_{4}$

Table S20. ${ }^{1} \mathrm{H}(500 \mathrm{MHz})$ and ${ }^{13} \mathrm{C}(125 \mathrm{MHz}) \mathrm{NMR}$ spectroscopic data for $\mathbf{~} \mathbf{4}$ in methanol- $d_{4}$

Table S21. ${ }^{13} \mathrm{C}$ NMR analysis of ficioilde $A(6)$ after feeding with $\left[1-{ }^{13} \mathrm{C}\right]$ sodium acetate, $\left[2-{ }^{13} \mathrm{C}\right]$ sodium acetate, and $\left[1,2-{ }^{13} \mathrm{C}_{2}\right]$ sodium acetate.

Table S22. HRESIMS data of new compounds.

\section{Supplementary Figures}

Figure S1. Generation of PfcclA and PfhdaA deletion strains.

Figure S2. Key HMBC (arrow) and 1H-1H COSY (blue boldline) correlations of 1-5 and 7-16.

Figure S3. The experiment ECD spectrum of $\mathbf{H 1}$ and previous reported ECD spectrum of ficipyrone A. Figure S4. DFT-optimized structures for low-energy conformers of $(3 R)-3$ at $\mathrm{B} 3 \mathrm{LYP} / 6-31+\mathrm{g}(\mathrm{d})$ level in methanol (PCM).

Figure S5. Experimental ECD and calculated spectra of 3.

Figure S6. Ortep diagram for the single crystal X-ray geometry of 6 .

Figure S7. $\Delta \delta_{S-R}$ values for the MTPA ester derivatives of 6, 9 and 11.

Figure S8. The relative configuration of 20 by comparison of coupling constants of 5 and 21.

Figure S9. DFT-optimized structures for low-energy conformers of $(1 S, 2 R, 5 S, 6 R)-5$ at B3LYP/6-31+g(d) level in methanol (PCM).

Figure S10. Experimental ECD and calculated spectra of 5 and 21.

Figure S11. Schematic illustration for hydrolysis of 1, 6, 7, 9-11.

Figures S12-S27. NMR spectra of compounds 1-16.

Figure S28. NMR spectra of compounds 21.

Figure S29. NMR analysis of H3 in methanol-d4.

Figure S30. NMR analysis of $\mathbf{H 4}$ in methanol-d4.

\section{Supplementary References}




\section{Supplementary methods}

Strains, media and growth conditions. Pestalotiopsis fici CGMCC3.15140 and its transformants (Table S4) were grown at $25{ }^{\circ} \mathrm{C}$ on Potato Dextrose Agar (PDA) or Potato Dextrose Broth (PDB) with appropriate antibiotics as required.

Escherichia coli strain DH5a and Agrobacterium tumefaciens AGL-1 were propagated in LB medium with appropriate antibiotics for plasmid DNA. For DNA manipulation in yeast, Saccharomyces cerevisiae strain BJ5464-NpgA (MATa ura3-52 his3-4200 leu2-41 trp1 pep4::HIS3 prb1 1.6R can1 GAL) was used as the host and grown on Yeast Extract Peptone Dextrose Medium (YPD). After transformation, S. cerevisiae was selected on synthetic dextrose complete medium (SDCt) with appropriate supplements corresponding to the auxotrophic markers ${ }^{1}$.

Gene cloning, plasmid construction and genetic manipulations. Plasmids used in this study are listed in Table S4. The oligonucleotide sequences for PCR primers are given in Table S5. PCR amplification was carried out using the TransStart $^{\circledR}$ FastPfu DNA polymerase (Transgene Biotech) on a $\mathrm{T}_{100}{ }^{\mathrm{TM}}$ Thermal cycler from Bio-Rad. P. fici DNA was isolated using the previously described method ${ }^{2}$.

Two steps were carried out for the creation of deletion strains of PfcclA (PFICl_05127). Firstly, the deletion cassette of PfcclA was constructed via a yeast recombination strategy. Briefly, the marker gene hygromycin B was amplified by using pDHt-hyg (Table S5) as template. Around $1 \mathrm{~kb}$ fragments upstream and downstream of the target gene PfcclA were amplified from genomic DNA of $P$. fici using designated primers (Table S5), respectively. These three amplified PCR fragments and linearized plasmid pXW06 (Table S4) were then purified with a EasyPure ${ }^{\circledR}$ Quick Gel Extraction Kit (Transgene Biotech), quantified, and assembled in yeast to give plasmid pYXW15.1 (Table S4). Secondly, the pYXW15.1 with the knock-out cassette was 
excised using the restriction enzymes $\mathrm{Kpnl}-\mathrm{Pacl}$ and inserted into binary vector pAG1-H3 to give pYXW25.1. All plasmids were confirmed by restriction enzyme digestion.

Pestalotiopsis fici transformation was performed via the ATMT method as described previously ${ }^{1}$. Plasmid pYXW25.1 was transformed into A. tumefaciens AGL-1. Hygromycin B resistant colonies were selected after being cultured on PDA at $28{ }^{\circ} \mathrm{C}$ for 5 days. The disruption mutants were verified by using diagnostic PCR with primers inside and outside the corresponding gene (Table S5).

For creation of PfhdaA (PFICl_08988) deletion strains, the deletion cassette of PfhdaA was constructed by using the same strategy as described above for PfhdaA to give plasmid pYXW16.1 (Table S4) and confirmed by restriction enzyme digestion. The conidia were germinated in TG medium (tryptone $10 \mathrm{~g} \mathrm{I}^{-1}$, glucose $100 \mathrm{~g} \mathrm{l}^{-1}$ ) for 30 hours at $28^{\circ} \mathrm{C}$ and $200 \mathrm{rpm}$ shaking. Mycelia were harvested and broken down in a TissueLyser at $70 \mathrm{~Hz}$ for $1 \mathrm{~min}$. Mycelia were cultured in $100 \mathrm{ml} \mathrm{TG}$ medium at $28^{\circ} \mathrm{C}$ with $200 \mathrm{rpm}$ shaking overnight (10h) and then digested in lysing enzyme $(30 \mathrm{mg} / \mathrm{ml})$ solution for 6 hours. The protoplasts were collected as described previously ${ }^{3}$. Transformation of $P$. fici was performed by the polyethylene glycol (PEG)-mediated fusion of protoplasts. The deletion cassette was amplified using primers K08988_5f_F and K08988_3f_R and the template pYXW16. Hygromycin B resistant colonies were selected after culturing on PDA at $28{ }^{\circ} \mathrm{C}$ for 5 days. The disruption mutants were verified by using diagnostic PCR with primers inside and outside the corresponding gene (Table S5).

Chemical analysis and characterization of compounds. For quantification of compounds regulated by PfCclA and PfHdaA, WT, $\triangle P f c c l A$ and $\triangle P h d a A$ strains were grown on rice media at $25{ }^{\circ} \mathrm{C}$ for 14 days. The material was extracted with $100 \mathrm{ml}$ of methanol/ethyl acetate/ acetic acid (MEA: 89:10:1) with 1 hour sonication at room temperature. The extract was evaporated in vacuo to yield a residue, which was suspended in $2 \mathrm{ml} \mathrm{MeOH}$. For PDA media, the same cultures were extracted at 7 days for secondary metabolite assessment. The compounds 6, 17, 
18, 19, 20 and 21 were used as standards (Figure S2) for HPLC analysis. The fold differences of these compounds between WT and mutants were calculated according to the following formula: [Area (Sample)-Area (Blank)]/[Area (WT)-Area (Blank)] $]^{4}$

Analytical HPLC was conducted with a Waters HPLC system (Waters e2695, Waters 2998, Photodiode Array Detector) using an ODS column $(\mathrm{C} 18,250 \times 4.6 \mathrm{~mm}$, YMC Pak, $5 \mu \mathrm{m})$ with a flow rate of $1 \mathrm{~mL} / \mathrm{min}$. Fresh extracts were dissolved in methanol ( $5 \mathrm{mg} / \mathrm{mL})$ before separated on a linear gradient of $\mathrm{MeOH}: \mathrm{H}_{2} \mathrm{O}(0.1 \%$ formic acid $)$ at a flow rate of $1 \mathrm{~mL} / \mathrm{min}$. Fresh extracts from $\triangle P f c c l A$ mutants were detected for 60 min using a linear gradient of $5 \%$ to $100 \%(0-45$ $\min$ ), $100 \% \mathrm{MeOH}(45-55 \mathrm{~min}), 5 \% \mathrm{MeOH}$ (55.01-60 min). Fresh extracts from $\triangle P f h d a A$ mutants were detected for 30 min using a linear gradient of $20 \%$ to $100 \%(0-20 \mathrm{~min}), 100 \%$ $\mathrm{MeOH}(20-25 \mathrm{~min}), 20 \% \mathrm{MeOH}$ (25.01-30 min). The targeted peaks in HPLC fingerprint were pointed out by comparing retention time with corresponding standard samples.

Purification of compounds $1-4$ and $17-20$ from $\triangle P f c c \mid A$ mutant. $\triangle P f c c l A$ mutant was cultured under static conditions at $28{ }^{\circ} \mathrm{C}$ in $500 \mathrm{~mL}$ Erlenmeyer flasks containing rice media (rice $40 \mathrm{~g}$, water $60 \mathrm{~mL}$ ). After 14 days of cultivation, the fermentation from 80 flasks was extracted three times with ethyl acetate. The organic phase was evaporated to dryness under reduced pressure to afford the residue $(36 \mathrm{~g})$. The organic extract was subjected to vacuum liquid chromatography over a silica gel column using a gradient elution with $\mathrm{CH}_{2} \mathrm{Cl}_{2}-\mathrm{MeOH}$ to give six fractions (fractions 1-6). Fraction 1 (14 g) eluted with $\mathrm{CH}_{2} \mathrm{Cl}_{2}$ was applied on a $\mathrm{C}-18$ ODS column using a stepped gradient elution of $\mathrm{MeOH}-\mathrm{H}_{2} \mathrm{O}$ yielding eight subfractions (fractions 1.11.8). The subfraction 1.6 (650 mg) was purified by Sephadex $\mathrm{LH}-20$ eluted with $\mathrm{MeOH}$ to give 7 subfractions (Fr.1.6.1-1.6.7). The fraction 1.6.1 (150 mg) was further purified by semipreparative HPLC $\left(75: 25 \mathrm{CH}_{3} \mathrm{OH} / \mathrm{H}_{2} \mathrm{O}, 0.1 \%\right.$ formic acid $\left.3 \mathrm{~mL} / \mathrm{min}\right)$ to afford $1\left(1.6 \mathrm{mg}, t_{\mathrm{R}} 13.5\right.$ $\mathrm{min}), 3\left(1.6 \mathrm{mg}, t_{\mathrm{R}} 20.3 \mathrm{~min}\right)$, respectively. The compounds, 4 (4.5 mg, $\left.t_{\mathrm{R}} 15.1 \mathrm{~min}\right), 2$ (1.0 mg, $\left.t_{\mathrm{R}} 20.1 \mathrm{~min}\right)$, were obtained by semi-HPLC $\left(80: 20 \mathrm{CH}_{3} \mathrm{OH} / \mathrm{H}_{2} \mathrm{O}, 0.1 \%\right.$ formic acid $\left.3 \mathrm{~mL} / \mathrm{min}\right)$. 
Compounds 17-20 were isolated and identified by comparing retention time with standard samples. HR-ESI-MS data of compounds 1 to $\mathbf{4}$ were shown in Table S22.

Pestaloficiol T (1), colorless oil. [a $]^{25}{ }_{\mathrm{D}}-16\left(\begin{array}{cc}c & 0.1, \mathrm{MeOH}\end{array}\right) .{ }^{1} \mathrm{H}$ NMR $\left(500 \mathrm{MHz}\right.$, methanol- $\left.d_{4}\right)$ and ${ }^{13} \mathrm{C}$ NMR (125 MHz, methanol- $d_{4}$ ) see Table S6 and attached spectra.

Pestaloficiol U (2), colorless powder. ${ }^{1} \mathrm{H}$ NMR $\left(500 \mathrm{MHz}\right.$, methanol- $\left.d_{4}\right)$ and ${ }^{13} \mathrm{C}$ NMR (125 $\mathrm{MHz}$, methanol- $\left.d_{4}\right)$ see Table S7 and attached spectra.

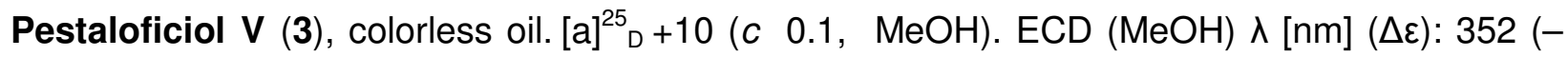
6.0), $307(+0.7), 260(-7.4), 241(-7.0), 215(-20) ;{ }^{1} \mathrm{H}$ NMR $\left(500 \mathrm{MHz}\right.$, methanol- $\left.d_{4}\right)$ and ${ }^{13} \mathrm{C}$ NMR (125 MHz, methanol- $\left.d_{4}\right)$ see Table S8 and attached spectra.

Ficipyrone C (4), colorless oil. ${ }^{1} \mathrm{H}$ NMR $\left(500 \mathrm{MHz}\right.$, methanol- $\left.d_{4}\right)$ and ${ }^{13} \mathrm{C} \mathrm{NMR} \mathrm{(125} \mathrm{MHz,}$ methanol- $\left.d_{4}\right)$ see Table S9 and attached spectra.

Purification of compounds 5-16 and 21 from $\triangle P f h d a A$ mutant. $\triangle P$ fhdaA mutant was cultivated in 10 liter of solid PDA media (BD) at $25^{\circ} \mathrm{C}$ for $7 \mathrm{~d}$. The PDA culture was cut into pieces and then extracted three times with ethyl acetate. The organic phase was evaporated to dryness under reduced pressure to afford the residue $(7.6 \mathrm{~g})$. The crude residue was applied on a C-18 ODS column using a stepped gradient elution of $\mathrm{MeOH}-\mathrm{H}_{2} \mathrm{O}$ yielding seven subfractions (fractions 1-10). Fraction 2 (eluting with $\mathrm{MeOH}: \mathrm{H}_{2} \mathrm{O}=25: 75$ ) was chromatographed on Sephadex $\mathrm{LH}-20(\mathrm{MeOH})$ and the targeted fraction 2.1 was further separated by HPLC (C-18 ODS) using a stepped gradient elution of $\mathrm{MeOH}-\mathrm{H}_{2} \mathrm{O}(5: 95$ to $100: 0,120 \mathrm{~min})$ to furnish eight subfractions 2.1.1-2.1.7. The subfration 2.1.1 was further purified by semi-preparative HPLC with a gradient of $\mathrm{H}_{2} \mathrm{O}$ and $\mathrm{CH}_{3} \mathrm{CN}$ (linear gradient of $15 \%$ to $45 \% \mathrm{CH}_{3} \mathrm{CN}$ over 30 min at 3 $\mathrm{mL} / \mathrm{min}$ ) to afford compounds $15\left(1.0 \mathrm{mg} t_{R}=11.2 \mathrm{~min}\right), 16\left(3.0 \mathrm{mg} t_{R}=12.2 \mathrm{~min}\right), 9\left(4.0 \mathrm{mg} t_{R}\right.$ $=13.0 \mathrm{~min}), 13\left(16.1 \mathrm{mg} t_{R}=14.5 \mathrm{~min}\right), 10\left(5.5 \mathrm{mg} t_{R}=16.8 \mathrm{~min}\right), 7\left(1.9 \mathrm{mg} t_{R}=17.3 \mathrm{~min}\right), 8$ (0.6 $\left.\mathrm{mg} t_{R}=20.1 \mathrm{~min}\right), 12\left(0.5 \mathrm{mg} t_{R}=23.6 \mathrm{~min}\right)$. The subfration 2.1 .5 was further purified by 
HPLC with a gradient of $\mathrm{H}_{2} \mathrm{O}$ and $\mathrm{CH}_{3} \mathrm{CN}$ (linear gradient of $25 \%$ to $55 \% \mathrm{CH}_{3} \mathrm{CN}$ over 30 min at $3 \mathrm{~mL} / \mathrm{min})$ to give compounds $11\left(15.7 \mathrm{mg}, t_{\mathrm{R}}=30.5 \mathrm{~min}\right)$ and $14\left(1.4 \mathrm{mg}, t_{\mathrm{R}}=16.5 \mathrm{~min}\right), 6(200$ $\left.\mathrm{mg}, t_{\mathrm{R}}=20.5 \mathrm{~min}\right), 5\left(0.7 \mathrm{mg}, t_{\mathrm{R}}=17.5 \mathrm{~min}\right), 21\left(70 \mathrm{mg}, t_{\mathrm{R}}=18.5 \mathrm{~min}\right)$. HR-ESI-MS data of compounds 5 , and 7 to 16 were shown in Table S22.

Pestaloficiol W (5), colorless oil. $[a]^{25}-10\left(c \quad 0.1, \quad \mathrm{CHCl}_{3}\right)$. ECD $(\mathrm{MeOH}) \lambda[\mathrm{nm}](\Delta \varepsilon): 308$ (+0.8), $270(-0.4), 232(+3.2), 206(-1.5) ;{ }^{1} \mathrm{H}$ NMR $\left(500 \mathrm{MHz}\right.$, methanol- $\left.d_{4}\right)$ and ${ }^{13} \mathrm{C}$ NMR (125 $\mathrm{MHz}$, methanol- $d_{4}$ ) see Table S18 and attached spectra.

Ficiolide A (6), colorless oil. $[\mathrm{a}]^{25}{ }_{\mathrm{D}}-10.9\left(\mathrm{c} \quad 0.1, \mathrm{CHCl}_{3}\right) .{ }^{1} \mathrm{H}$ NMR $\left(500 \mathrm{MHz}\right.$, methanol- $\left.d_{4}\right)$ and ${ }^{13} \mathrm{C}$ NMR $\left(125 \mathrm{MHz}\right.$, methanol- $\left.d_{4}\right)$ see Table S10 and attached spectra. ESI-MS $\left.[\mathrm{M}+\mathrm{H}]\right]^{+} \mathrm{m} / \mathrm{z}$ 313.1.

Ficiolide B (7), colorless oil. $[\mathrm{a}]_{\mathrm{D}}^{25}-47(\mathrm{c} \quad 0.1, \mathrm{MeOH}) .{ }^{1} \mathrm{H}$ NMR $\left(500 \mathrm{MHz}\right.$, methanol- $\left.d_{4}\right)$ and ${ }^{13} \mathrm{C}$ NMR (125 MHz, methanol- $d_{4}$ ) see Table S11 and attached spectra.

Ficiolide C (8), colorless oil. $[\mathrm{a}]_{\mathrm{D}}^{25}-42(\mathrm{c} \quad 0.1, \mathrm{MeOH}) .{ }^{1} \mathrm{H}$ NMR $\left(500 \mathrm{MHz}\right.$, methanol- $\left.d_{4}\right)$ and ${ }^{13} \mathrm{C}$ NMR $\left(125 \mathrm{MHz}\right.$, methanol- $\left.d_{4}\right)$ see Table $\mathrm{S} 11$ and attached spectra.

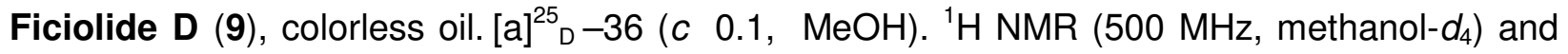
${ }^{13} \mathrm{C}$ NMR $\left(125 \mathrm{MHz}\right.$, methanol- $\left.d_{4}\right)$ see Table $\mathrm{S} 12$ and attached spectra.

Ficiolide E (10), colorless oil. $[a]^{25}-51(c \quad 0.1, \mathrm{MeOH}) .{ }^{1} \mathrm{H}$ NMR $\left(500 \mathrm{MHz}\right.$, methanol- $\left.d_{4}\right)$ and ${ }^{13} \mathrm{C}$ NMR (125 MHz, methanol- $d_{4}$ ) see Table $\mathrm{S} 12$ and attached spectra.

Ficiolide $\mathbf{F}$ (11), colorless oil. $[\mathrm{a}]_{\mathrm{D}}^{25}-50\left(\mathrm{c} \quad 0.1, \mathrm{CHCl}_{3}\right) .{ }^{1} \mathrm{H}$ NMR $\left(500 \mathrm{MHz}\right.$, methanol- $\left.d_{4}\right)$ and ${ }^{13} \mathrm{C}$ NMR $\left(125 \mathrm{MHz}\right.$, methanol- $\left.d_{4}\right)$ see Table $\mathrm{S} 13$ and attached spectra.

Ficiolide G (12), colorless oil. [a] ${ }^{25}-33\left(\begin{array}{ccc}c & 0.1, & \mathrm{MeOH}\end{array}\right) .{ }^{1} \mathrm{H}$ NMR $\left(500 \mathrm{MHz}\right.$, methanol- $\left.d_{4}\right)$ and ${ }^{13} \mathrm{C}$ NMR $\left(125 \mathrm{MHz}\right.$, methanol- $d_{4}$ ) see Table $\mathrm{S} 14$ and attached spectra. 
Ficiolide H (13), colorless oil. $[\mathrm{a}]^{25}{ }_{\mathrm{D}}-156(\mathrm{c} \quad 0.1, \mathrm{MeOH}) .{ }^{1} \mathrm{H}$ NMR $\left(500 \mathrm{MHz}\right.$, methanol- $\left.d_{4}\right)$ and ${ }^{13} \mathrm{C}$ NMR $\left(125 \mathrm{MHz}\right.$, methanol- $d_{4}$ ) see Table $\mathrm{S} 15$ and attached spectra.

Ficiolide I (14), colorless oil. [a] ${ }_{\mathrm{D}}^{25}+50.6(\mathrm{c} \quad 0.1, \mathrm{MeOH}) .{ }^{1} \mathrm{H}$ NMR $\left(500 \mathrm{MHz}\right.$, methanol- $\left.d_{4}\right)$ and ${ }^{13} \mathrm{C}$ NMR $\left(125 \mathrm{MHz}\right.$, methanol- $\left.d_{4}\right)$ see Table $\mathrm{S} 15$ and attached spectra.

Ficiolide J (15), colorless oil. [a $]^{25}-20\left(\begin{array}{ccc}c & 0.1, & \mathrm{MeOH}\end{array}\right) .{ }^{1} \mathrm{H}$ NMR $\left(500 \mathrm{MHz}\right.$, methanol- $\left.d_{4}\right)$ and ${ }^{13} \mathrm{C}$ NMR (125 MHz, methanol- $d_{4}$ ) see Table S16 and attached spectra.

Ficiolide K (16), colorless oil. [a $]^{25}{ }_{\mathrm{D}}-29$ (c $\left.0.1, \mathrm{MeOH}\right) .{ }^{1} \mathrm{H}$ NMR $\left(500 \mathrm{MHz}\right.$, methanol- $\left.d_{4}\right)$ and ${ }^{13} \mathrm{C}$ NMR (125 MHz, methanol- $d_{4}$ ) see Table S17 and attached spectra.

Asperpentyn (21), ECD (MeOH) $\lambda[\mathrm{nm}](\Delta \varepsilon)$ : $308(-0.5), 260(-4.7), 220(+2.1), 205(-4.3) ;{ }^{1} \mathrm{H}$ NMR (500 MHz, methanol- $d_{4}$ ) and ${ }^{13} \mathrm{C}$ NMR (125 MHz, methanol- $\left.d_{4}\right)$ see Table S18 and attached spectra.

NMR of H3. ${ }^{1} \mathrm{H}$ NMR $\left(500 \mathrm{MHz}\right.$, methanol- $\left.d_{4}\right)$ and ${ }^{13} \mathrm{C}$ NMR $\left(125 \mathrm{MHz}\right.$, methanol- $\left.d_{4}\right)$ see Table S19 and attached spectra.

NMR of H4. ${ }^{1} \mathrm{H}$ NMR $\left(500 \mathrm{MHz}\right.$, methanol- $\left.d_{4}\right)$ and ${ }^{13} \mathrm{C}$ NMR $\left(125 \mathrm{MHz}\right.$, methanol- $\left.d_{4}\right)$ see Table S20 and attached spectra.

ECD of H1 and ficipyrone A. H1: ECD $\lambda[\mathrm{nm}](\Delta \varepsilon):(c=0.2 \mathrm{mg} / \mathrm{mL}, \mathrm{MeOH}): 282(-1.99), 215(+$ 0.61). ECD spectrum of ficipyrone A see described ${ }^{5}$.

Analytical methods and equipment overview. CD spectra were measured on JASCO J-815 spectropolarimeter. NMR spectra $\left({ }^{1} \mathrm{H},{ }^{13} \mathrm{C}, \mathrm{HMBC},{ }^{1} \mathrm{H}-{ }^{1} \mathrm{H}\right.$ COSY, HSQC) were recorded on a Bruker Avance-500 spectrometer using TMS as internal standard, and chemical shifts were recorded as $\delta$ values. HR-ESI-MS utilized on an Agilent Accurate-Mass-QTOF LC/MS 6520 instrument. X-ray diffraction was realized on an Xcalibur, Eos, Gemini instrument at $293 \mathrm{~K}$ using Cu Ka radiation. HPLC analysis was performed on a Waters HPLC system (Waters e2695, Waters 2998, Photodiode Array Detector) using an ODS column (C18, $250 \times 4.6 \mathrm{~mm}$, YMC Pak, 
$5 \mu \mathrm{m}$ ) with a flow rate of $1 \mathrm{~mL} / \mathrm{min}$. Semi-prepartive HPLC was performed using an ODS column [HPLC (YMC-Pack ODS-A, $10 \times 250 \mathrm{~mm}, 5 \mu \mathrm{m}, 3 \mathrm{~mL} / \mathrm{min})]$. Column chromatography (CC) were performed with silica gel (200-300 mesh, Qingdao Marine Chemical Inc., Qingdao, China), and Sephadex LH-20 (Amersham Biosciences), respectively.

Statistical analysis. For statistical analyses, data were analyzed using the GraphPad Instate software package, version 5.01 (GraphPad software Inc) according to the Tukey-Kramer multiple comparison test at $\mathrm{P} \leq 0.05$. Mean values with asterisks are statistically significant.

X-ray Crystallographic Analysis of Compounds 6. Colorless crystals of 6 were obtained in the mixed solvents of Methanol/ $\mathrm{H}_{2} \mathrm{O}$, and Crystallographic data (excluding structure factors) for 6 (Cu Ka radiation), has been deposited with the Cambridge Crystallographic Data Center as supplementary publication numbers CCDC 1414142 . These data can be obtained free of charge from The Cambridge Crystallographic Data Centre via www.ccdc.cam.ac.uk/data request/cif.

Crystal Data for 6. Orthorhombic, $\mathrm{C}_{16} \mathrm{H}_{24} \mathrm{O}_{6}$, space group $\mathrm{P} 2{ }_{1} 2_{1} 2_{1}$ (no. 19), $a=9.9129(4) \AA, b=$ $10.5346(4) \AA, c=16.4552(9) \AA, V=1718.39$ (13) $\AA^{3}, Z=4, T=293(2) \mathrm{K}, \mu(\mathrm{CuK \alpha})=0.763 \mathrm{~mm}^{-}$ ${ }^{1}, D_{\text {calcd }}=1.2074(1) \mathrm{g} / \mathrm{cm}^{3}, 2556$ unique $\left(R_{\text {int }}=0.0301\right)$ which were used in all calculations. The final $R_{1}$ was $0.0519[\mathrm{I}>2 \sigma(\mathrm{I})]$ and $w R_{2}$ was 0.1209 (all data). Flack parameter 0.00 .

Computation Section ${ }^{6}$. Conformational searches were run employing the "systematic" procedure implemented in Spartan'14, using MMFF (Merck molecular force field). All MMFF minima were reoptimized with DFT calculations at the B3LYP/6-31+G(d) level using the Gaussian09 program. The geometry was optimized starting from various initial conformations, with vibrational frequency calculations confirming the presence of minima. Time-dependent DFT calculations were performed on 4 lowest-energy conformations ( $>5 \%$ population) for each configuration using 30 excited states, using a polarizable continuum model (PCM) in $\mathrm{MeOH}$ for 5 and 3. ECD spectra were generated using the program SpecDis by applying a Gaussian band shape with $0.28 \mathrm{eV}$ (for 5 ) and 0.26 (for $\mathbf{3}$ ) width, from dipole-length rotational strengths. The 
dipole velocity forms yielded negligible differences. The spectra of the conformers were combined using Boltzmann weighting, with the lowest-energy conformations accounting for about $99 \%$ of the weights.

Feeding Experiments with $\left[1-{ }^{13} \mathrm{C}\right]$ sodium acetate, $\left[2-{ }^{13} \mathrm{C}\right]$ sodium acetate and $\left[1,2-{ }^{13} \mathrm{C}_{2}\right]$ sodium acetate. For the isotope labeling experiments, $500 \mathrm{mg}$ of $\left[1-{ }^{-13} \mathrm{C}\right]$ sodium acetate, $\left[2-{ }^{13} \mathrm{C}\right]$ sodium acetate, or $350 \mathrm{mg}$ of $\left[1,2-{ }^{13} \mathrm{C}_{2}\right]$ sodium acetate were dissolved in $1 \mathrm{~mL}$ of water and filter sterilized, respectively. The aqueous solutions of isotope labled compounds was add to $1 \mathrm{~L}$ of unset PDA medium which was then averagely divided into 50 culture dishes $(90 \mathrm{~mm}) .5 \mu \mathrm{L}$ of Spore suspension (concentration of approximately $1 \times 10^{7}$ spore $/ \mathrm{mL}$ ) was inoculated to each culture dish and cultured at $25{ }^{\circ} \mathrm{C}$ for 7 days. The PDA medium was harvested and cut into pieces followed by extract with $1 \mathrm{~L}$ of MEA (x 3 times). Purification of the target ficioilde $\mathrm{A}$ was achieved by sephadex LH-20 column chromatography and semi-preparative reversed-phase HPLC to afford 5-20 mg of each labeled compound (Table S21).

Preparation of MTPA Esters of $6,10,11 .(R)$-MTPACl $(8 \mu \mathrm{L})$ was added to a deuterated pyridine $(500 \mu \mathrm{L})$ solution containing compound $6(0.8 \mathrm{mg})$ in NMR tube at room temperature under a nitrogen atmosphere for $12 \mathrm{~h}$. The organic phase was then evaporated to dryness and separated by semi-preparative HPLC to afford the (S)-MTPA ester-6. In a similar manner, the (R)-MTPA ester-6 was prepared from 6 using (S)-MTPACl.

The same procedure was used for the preparation of the $(R)$ - or (S)-MTPA diesters-10 and $(R)$ - or (S)-MTPA esters-11 obtained from 10 and 11, respectively. The mixture was directly analyzed by ${ }^{1} \mathrm{H}$ NMR without purification.

Hydrolysis of 1, 6, 7, 9-11. Within solution ( $0.8 \mathrm{~mL}$ for $1 ; 1.5 \mathrm{~mL}$ for $\mathbf{6}, \mathbf{1 1} ; 0.5 \mathrm{~mL}$ for $\mathbf{7}, \mathbf{9}, \mathbf{1 0}$;) of compounds in a $\mathrm{CH}_{2} \mathrm{Cl}_{2} / \mathrm{MeOH}\left(90: 10\right.$, v:v) mixture, $\mathrm{CH}_{3} \mathrm{ONa}$ (0.8 mg for 1; $3.5 \mathrm{mg}$ for 6, 11; $0.5 \mathrm{mg}$ for $9,10,7$;) was added and then stirred for $2-5 \mathrm{~h}$ at $0{ }^{\circ} \mathrm{C}$. Ion exchange resin added to 
the reaction mixture and then filtered the mixture to obtain the supernatant liquid (Figure S11). The organic solvent was purified by semi-preparative RP-HPLC to yield hydrolytic products.

The structures of $\mathbf{H} \mathbf{1}$ and $\mathbf{H} \mathbf{2}$ were determined by comparing retention times with the corresponding standards. The absolute configuration of $\mathbf{H 2}$ was established based on the comparison ECD with ficipyrone A. The structures of $\mathbf{H 3}$ and $\mathbf{H} \mathbf{4}$ were determined by NMR and HR-ESI-MS (see Table S20.)

\section{Supplementary Tables}


Table S1. PKSs encoded in Pestalotiopsis fici genome

\begin{tabular}{|c|c|c|c|c|c|}
\hline No. & Gene ID & Cluster Number & Module organization & Homolog accession number & Closest BLAST hit (s) (coverage/identity) \\
\hline & NR-PKSs & & & & \\
\hline 1 & PFICI_00366 & 18 & KS-AT-DH-ACP-MT & XP_003071593.1 & Coccidioides posadasii C735 delta SOWgp (99\%/48\%) \\
\hline 2 & PFICI_03986 & 30 & KS-AT-ACP-ACP-MT & XP_007596699.1 & Colletotrichum fioriniae PJ7(99\%/48\%) \\
\hline 3 & PFICI_07101 & 45 & KS-AT-ACP-ACP-TE & AGT56219.1 & Pestalotiopsis malicola $(100 \% / 95 \%)$ \\
\hline 4 & $\begin{array}{l}\text { PFICI_10824 } \\
\text { HR-PKSs }\end{array}$ & 66 & KS-AT-ACP & ESZ98980.1 & Sclerotinia borealis F-4157 (98\%/66\%) \\
\hline 5 & PFICI_00149 & 16 & KS-AT-DH-MT-ER-KR-ACP & EME41518.1 & Dothistroma septosporum NZE10 (53\%) \\
\hline 6 & PFICI_00294 & 17 & KS-AT-DH-MT-KR-ACP & KID84093.1 & Metarhizium guizhouense ARSEF 977 (99\%/58\%) \\
\hline 7 & PCICI_00493 & 19 & KS-AT-DH-MT-ER-KR-ACP & CRG92723.1 & Talaromyces islandicus $(99 \% / 57 \%)$ \\
\hline 8 & PFICI_01588 & 22 & KS-AT-DH-MT-ER-KR-ACP & XP_007278670.1 & Colletotrichum gloeosporioides Nara gc5 (99\%/45\%) \\
\hline 9 & PFICI_02353 & 25 & KS-AT-DH-MT-ER-KR-ACP & CRL19370.1 & Penicillium camemberti (98\%/48\%) \\
\hline 10 & PFICI_02665 & 27 & KS-AT-DH-MT-ER-KR-ACP & KND86893.1 & Tolypocladium ophioglossoides CBS 100239 (98\%/58\%) \\
\hline 11 & PFICI_06068 & 39 & KS-AT-DH-ER-KR-ACP & EFX00060.1 & Grosmannia clavigera kw1407 (98\%/64\%) \\
\hline 12 & PFICI_06561 & 42 & KS-AT-DH-ACP-ACP-TD & AAR92210.1 & Fusarium verticillioides (99\%/59\%) \\
\hline 13 & PFICI_07941 & 49 & KS-AT-DH-MT-KR-ACP-C & XP_001274946.1 & Aspergillus clavatus NRRL 1 (99\%/74\%) \\
\hline 14 & PFICI_07942 & 49 & KS-AT-DH-MT-ER-KR-ACP & XP_003350293.1 & Sordaria macrospora k-hell (100\%/78\%) \\
\hline 15 & PFICI_07954 & 50 & KS-AT-DH-ER-KR-ACP & XP_003350293.1 & Glomerella graminicola M1.001 (99\%/61\%) \\
\hline 16 & PFICI_07957 & 50 & KS-ER-KR-ACP & AEE65377.1 & Peltigera membranacea $(98 \% / 53 \%)$ \\
\hline 17 & PFICI_08471 & 53 & KS-AT-DH-KR-ACP & XP_011395279.1 & Neurospora crassa OR74A(98\%/49\%) \\
\hline 18 & PFICI_08706 & 55 & KS-AT-DH-ER-KR-ACP & EYE95336.1 & Aspergillus ruber CBS 135680 (98\%/51\%) \\
\hline 19 & PFICI_09718 & 59 & KS-AT-DH-ER-KR-ACP & ESZ94274.1 & Sclerotinia borealis F-4157 (99\%/33\%) \\
\hline 20 & PFICI_10088 & 62 & KS-AT-DH-ACP-MT & CCD46020.1 & Botrytis cinerea $\mathrm{T} 4(84 \% / 59 \%)$ \\
\hline 21 & PFICI_10144 & 63 & KS-AT-DH-MT-ER-KR-ACP & KOS22233.1 & Escovopsis weberi $(99 \% / 48 \%)$ \\
\hline 22 & PFICI_10987 & 67 & KS-AT-DH-ER-KR-ACP & KOP48046.1 & Madurella mycetomatis (98\%/62\%) \\
\hline 23 & PFICI_11984 & 73 & KS-AT-DH-MT-ER-KR-ACP & KOP50079.1 & Madurella mycetomatis (99\%/48\%) \\
\hline 24 & PFICI_12429 & 2 & KS-AT-DH-ER-KR-ACP & BAM77409.1 & Talaromyces purpureogenus (98\%/59\%) \\
\hline 25 & PFICI_12549 & 4 & KS-AT-DH-MT-ER-KR-ACP & EQB57236.1 & Colletotrichum gloeosporioides Cg-14 (99\%/51\%) \\
\hline 26 & PFICI_12888 & 6 & KS-AT-DH-MT-ER-KR-ACP & XP_001274957.1 & Aspergillus clavatus NRRL 1 (99\%/45\%) \\
\hline 27 & PFICI_12928 & 7 & KS-AT-DH-KR-ACP & AIW00670.1 & Parastagonospora nodorum (98\%/65\%) \\
\hline 28 & PFICI_13417 & 9 & KS-AT-DH-MT-ER-KR-ACP & XP_002487607.1 & Talaromyces stipitatus ATCC 10500 (99\%/47\%) \\
\hline 29 & PFICI_15056 & 13 & KS-AT-DH-ER-KR-ACP & ADF28668.2 & Peltigera membranacea $(98 \% / 46 \%)$ \\
\hline 30 & PFICI_15221 & 14 & KS-AT-DH-MT-KR-ACP & XP_007799223.1 & Eutypa lata UCREL1(98\%/45\%) \\
\hline
\end{tabular}


Table S2. NRPS, NRPS-like encoded in Pestalotiopsis fici genome

\begin{tabular}{|c|c|c|c|c|c|}
\hline No. & Gene ID & $\begin{array}{l}\text { Cluster } \\
\text { Number } \\
\end{array}$ & Module organization & Homolog accession number & Closest BLAST hit (s) (coverage/identity) \\
\hline & NRPS & & & & \\
\hline 1 & PFICI_00152 & 16 & $\begin{array}{l}\text { T-C-A-T-C-A-T-C-A-T-C-A-T-C-A-T- } \\
\text { C }\end{array}$ & GAM84991.1 & fungal sp. No.11243(98\%/41\%) \\
\hline 2 & PFICI_01040 & 20 & $\begin{array}{l}\text { A-T-C-A-T-E-C-A-T-C-A-T-E-C-A-T- } \\
\text { C-A-T-E-C-A-T-C }\end{array}$ & EXU95985.1 & Metarhizium robertsii $(99 \% / 33 \%)$ \\
\hline 3 & PFICI_02081 & 23 & A-T-TD-KR & XP_003716672.1 & Magnaporthe oryzae $70-15(100 \% / 75 \%)$ \\
\hline 4 & PFICI_02307 & 24 & A-T-C & KDN65336.1 & Colletotrichum sublineola $(99 \% / 34 \%)$ \\
\hline 5 & PFICI_02313 & 24 & T-C-A-T-C & EIT81855.1 & Aspergillus oryzae 3.042 (97\%/48\%) \\
\hline 6 & PFICI_02554 & 26 & A-T-C-A-T-C-A-T-C-A-T-E & XP_003713094.1 & Magnaporthe oryzae $70-15(98 \% / 48 \%)$ \\
\hline 7 & PFICI_05764 & 38 & A-T-C-A-T-C & KHO01329.1 & etarhizium album ARSEF 1941 (96\%/41\%) \\
\hline 8 & PFICI_08385 & 52 & T-C-C-A-C & XP_003654811.1 & Thielavia terrestris NRRL 8126 (92\%/41\%) \\
\hline 9 & PFICI_09514 & 57 & A-T-C-C-A-T-E-TE & KOC16988.1 & Aspergillus flavus AF70(95\%/34\%) \\
\hline 10 & PFICI_09663 & 58 & T-C-A-T-C & CCD52775.1 & Botrytis cinerea T4 (99\%/41\%) \\
\hline 11 & PFICI_10656 & 65 & A-T-C-A-T-C-T-C-A-T-C-T-C-T-C & XP_003661000.1 & $\begin{array}{l}\text { Myceliophthora thermophila ATCC } 42464 \\
(99 \% / 44 \%)\end{array}$ \\
\hline 12 & PFICI_11786 & 72 & C-A-T-E-C-A-T-C-A-T-C-A-T-E & EQL02707.1 & Ophiocordyceps sinensis CO18(99\%/65\%) \\
\hline 13 & PFICI_13707 & 10 & A-T-C & GAO81963.1 & Neosartorya udagawae (99\%/35\%) \\
\hline 14 & $\begin{array}{l}\text { PFICI_14616 } \\
\text { NRPS-like }\end{array}$ & 12 & A-T-C-T-C & XP_007795002.1 & Eutypa lata UCREL1 (98\%/67\%) \\
\hline 15 & PFICI_00364 & 18 & A-NAD & XP_007596707.1 & Colletotrichum fioriniae PJ7(97\%/59\%) \\
\hline 16 & PFICI_02312 & 24 & $\mathrm{~A}$ & XP_001817984.2 & Aspergillus oryzae RIB40 (98\%/55\%) \\
\hline 17 & PFICI_03128 & 28 & A-ACP & КKР07640.1 & Trichoderma harzianum (99\%/53\%) \\
\hline 18 & PFICI_05086 & 35 & A-NAD & EFX03079.1 & Grosmannia clavigera kw1407 (98\%/50\%) \\
\hline 19 & PFICI_05312 & 36 & A-T-NAD & EFX03079.1 & Grosmannia clavigera kw1407 (99\%/57\%) \\
\hline 20 & PFICI_05398 & 37 & A-T-NAD & KLO82681.1 & Fusarium fujikuroi (98\%/59\%) \\
\hline 21 & PFICI_06834 & 44 & A-T-NAD & XP_013273402.1 & Rasamsonia emersonii CBS 393.64 (95\%/36\%) \\
\hline 22 & PFICI_08077 & 51 & A-NAD & KMM71381.1 & Coccidioides posadasii RMSCC 3488 (100\%/45\%) \\
\hline 23 & PFICI_08544 & 54 & A-T-NAD & ENH86099.1 & $\begin{array}{l}\text { Colletotrichum orbiculare MAFF } 240422 \\
(96 \% / 61 \%)\end{array}$ \\
\hline 24 & PFICI_08738 & 56 & A-T-NAD & ALC79549.1 & Didymella rabiei $(99 \% / 66 \%)$ \\
\hline 25 & PFICI_10568 & 64 & A-T-NAD & XP_007826411.2 & Metarhizium robertsii ARSEF 23(99\%/48\%) \\
\hline 26 & PFICI_11332 & 70 & A-T-NAD & KOP46026.1 & Madurella mycetomatis (98\%/56\%) \\
\hline 27 & PFICI_11756 & 71 & A-T-NAD & XP_965396.1 & Neurospora crassa OR74A(99\%/76\%) \\
\hline 28 & PFICI_12508 & 3 & A-T-NAD & XP_008097819.1 & Colletotrichum graminicola M1.001(99\%/61\%) \\
\hline 29 & PFICI_12763 & 5 & A-T-NAD & ССТ74830.1 & Fusarium fujikuroi IMI 58289 (99\%/48\%) \\
\hline 30 & PFICI_13208 & 8 & A-T-TE & GAA88295.1 & Aspergillus kawachii IFO 4308(99\%/44\%) \\
\hline
\end{tabular}


Table S3. Hybrid PKS-NRPS and Terpene encoded in Pestalotiopsis fici genome

\begin{tabular}{|c|c|c|c|c|c|}
\hline No. & Gene ID & $\begin{array}{l}\text { Cluster } \\
\text { Number }\end{array}$ & Module organization & $\begin{array}{l}\text { Homolog accession } \\
\text { number }\end{array}$ & Closest BLAST hit (s) (coverage/identity) \\
\hline & NRPS-PKS & & & & \\
\hline 1 & PFICI_04360 & 32 & A-ACP-KS-AT-KR-ACP-TE & WP_006374567.1 & Streptomyces turgidiscabies $(99 \% / 41 \%)$ \\
\hline 2 & PFICI_06351 & 40 & A-PCP-KS-AT-KR-ACP-TD & XP_007815889.1 & Metarhizium acridum CQMa 102(99\%/53\%) \\
\hline 3 & PFICI_07789 & 48 & A-PCP-KS-AT-KR-ACP-TD & KOP46033.1 & Madurella mycetomatis( $98 \% / 64 \%)$ \\
\hline \multirow[t]{2}{*}{4} & PFICI_15331 & 15 & A-ACP-KS-AT-DH-KR-ACP-TE & WP_006374567.1 & Streptomyces turgidiscabies (99\%/40\%) \\
\hline & Terpene & & & & \\
\hline 1 & PFICI_01173 & 21 & terpene synthase & AHY23920.1 & Hypoxylon sp. E7406B(98\%/54\%) \\
\hline 2 & PFICI_03884 & 29 & SirD & GAA86868.1 & Aspergillus kawachii IFO 4308(95\%/48\%) \\
\hline 3 & PFICI_04042 & 31 & 7-dimethylallyltryptophan synthase & KOP43609.1 & Madurella mycetomatis (91\%/39\%) \\
\hline 4 & PFICI_04870 & 34 & terpenoid synthase & KIY69043.1 & $\begin{array}{l}\text { Cylindrobasidium torrendii FP15055 ss- } \\
10(91 \% / 43 \%)\end{array}$ \\
\hline 5 & PFICI_06391 & 41 & $\begin{array}{l}\text { geranylgeranyl pyrophosphate } \\
\text { synthetase }\end{array}$ & XP_011319033.1 & Fusarium graminearum $\mathrm{PH}-1$ (95\%/68) \\
\hline 6 & PFICI_06798 & 43 & squalene synthetase & XP_011395225.1 & Neurospora crassa OR74A(99\%/69\%) \\
\hline 7 & PFICI_07178 & 46 & terpene synthase & AHY23925.1 & Hypoxylon sp. E7406B (97\%/47\%) \\
\hline 8 & PFICI_07334 & 47 & terpene synthase & AHY23929.1 & Hypoxylon sp. E7406B(62\%/48\%) \\
\hline 9 & PFICI_09860 & 60 & squalene/phytoene synthase & XP_007596091.1 & Colletotrichum fioriniae $\mathrm{PJ} 7(99 \% / 58 \%)$ \\
\hline 10 & PFICI_11288 & 69 & pentalenene synthase & KNG89410.1 & Aspergillus nomius NRRL 13137(86\%/49\%) \\
\hline 11 & PFICI_12410 & 1 & retinol dehydrogenase 12 & CCF35689.1 & Colletotrichum higginsianum $(96 \% / 55 \%)$ \\
\hline 12 & PFICI_14247 & 11 & aromatic prenyltransferase & CCF39038.1 & Colletotrichum higginsianum (99\%/74\%) \\
\hline
\end{tabular}


6 Table S4. Fungal strains and plasmids used in this study

\begin{tabular}{lll}
\hline Strain/plasmid & Description & Reference \\
\hline Pestalotiopsis fici & Wild type & {$[3]$} \\
CGMCC3.15140 & A.tumefaciens strain & {$[1]$} \\
AGL-1 & $\Delta P F I C I$ 05127::hph & This study \\
TYXW7.1 & DFICI_08988::hph & This study \\
TYXW8.1 & binary donor vectors pAg1-H3 & {$[7]$} \\
pAG1-H3 & yeast recombination vector & {$[8]$} \\
pXW06 & PFICI_05127 deletion cassette in & This study \\
pYXW15 & pXW06 & \\
& PFICI_05127 deletion cassette in pAG1- & This study \\
pYXW25.1 & H3 & This study \\
pYXW16 & PFICI_08988 deletion cassette in & \\
& pXW06 & \\
\hline
\end{tabular}

$\mathrm{pXX}=$ plasmid, $\mathrm{TXX}=$ original transformant 
Table S5. PCR primer sets used in this study

\begin{tabular}{|c|c|c|}
\hline Name & Oligonucleotide sequence (5'-3') & Uses \\
\hline 05127RT_F & GAACCCAAACCCAGAACC & PFICl_05127 \\
\hline 05127RT_R & CGCATCCTCGAAGCTCATG & transformant screening \\
\hline K05127_5f_F & $\begin{array}{l}\text { ACTATCAACTATTAACTATATCGTAATACC } \\
\text { ATCATACACATGCCTTTCCGTTCC }\end{array}$ & 5 flanks amplification \\
\hline K05127_5f_ & CCTTCAATATCATCTTCTGTCGACCTCGA & \\
\hline K05127_3f_F & $\begin{array}{l}\text { CGATAAGCTTGATATCGAATTCCTGCAGC } \\
\text { CCGCGCCAAGGATGGTGAGATC }\end{array}$ & 3 flanks amplification \\
\hline $\begin{array}{l}\text { K05127_3f_ } \\
\text { R }\end{array}$ & $\begin{array}{l}\text { ACTTGATAATGGAAACTATAAATCGTGAA } \\
\text { GGCATGTTTGAGATGGTCCGCAAGTTC }\end{array}$ & \\
\hline $\begin{array}{l}05127 \_5 f \_E F \\
05127 \bar{T} \_ \text {R }\end{array}$ & CGCACAAGTTATCGTGCAC & $\begin{array}{l}\text { PFICl_05127 } \\
\text { transformant } 5 \mathrm{~F}\end{array}$ \\
\hline & CTATGTCAGGCACCCAGTC & screening \\
\hline $\begin{array}{l}\text { 05127T_F } \\
\text { 05127_3f_E }\end{array}$ & CTCGCCGATAGTGGAAACC & $\begin{array}{l}\text { PFICl_05127 } \\
\text { transformant 3F }\end{array}$ \\
\hline & GACGACCTCAACCTGTC & screening \\
\hline 08988RT_F & CCCTGGAAAGCCAGACAATATG & PFICI_08988 \\
\hline 08988RT_R & CTGGTAGTTGCGGATGACGTC & transformant screening \\
\hline K08988_5f_F & $\begin{array}{l}\text { ACTATCAACTATTAACTATATCGTAATACC } \\
\text { ATCATAGCAGCGGTAACTGAAACG }\end{array}$ & 5 flanks amplification \\
\hline $\begin{array}{l}\text { K08988_5f_- } \\
\text { R }\end{array}$ & $\begin{array}{l}\text { CCTTCAATATCATCTTCTGTCGACCTCGA } \\
\text { GGGAAGGAAGCCGAGCCATATG }\end{array}$ & \\
\hline K08988_3f_F & $\begin{array}{l}\text { CGATAAGCTTGATATCGAATTCCTGCAGC } \\
\text { CCGGTTGGAATGGAGGCTGGGATC }\end{array}$ & 3 flanks amplification \\
\hline $\begin{array}{l}\text { K08988_3f_ } \\
\mathrm{R}\end{array}$ & $\begin{array}{l}\text { ACTTGATAATGGAAACTATAAATCGTGAA } \\
\text { GGCATGTTTCATAGGTGGTTTGCTTGTGG } \\
\text { C }\end{array}$ & \\
\hline 08988 5f_EF & GAAGGTCCGTTGGTGATG & PFICl_08988 \\
\hline 08988T_R & GTCCTCGTTCCTGTCTGCTA & $\begin{array}{l}\text { transformant } 5 \mathrm{~F} \\
\text { screening }\end{array}$ \\
\hline $\begin{array}{l}\text { 08988T_F } \\
\text { 08988_3f_E } \\
R\end{array}$ & $\begin{array}{l}\text { GTCCAAGCAGCAAAGAGTG } \\
\text { GAGCGGCAATAGAATGAGG }\end{array}$ & $\begin{array}{l}\text { PFICl_08988 } \\
\text { transformant 3F } \\
\text { screening }\end{array}$ \\
\hline hyg_f & $\begin{array}{l}\text { CCTCGAGGTCGACAGAAGATGATATTGAA } \\
\text { GGGCGCAATTAACCCTCACTA }\end{array}$ & $\begin{array}{l}\text { Hygromycin B } \\
\text { amplification }\end{array}$ \\
\hline hyg_r & $\begin{array}{l}\text { CGGGCTGCAGGAATTCGATATCAAGCTTA } \\
\text { TCGCAGGGCTGGTGACGGAAT }\end{array}$ & \\
\hline
\end{tabular}


Table S6. ${ }^{1} \mathrm{H}(500 \mathrm{MHz})$ and ${ }^{13} \mathrm{C}(125 \mathrm{MHz})$ NMR spectroscopic data for 1 in Acetone$d_{6}$.

\begin{tabular}{|c|c|c|c|c|c|}
\hline \multicolumn{6}{|c|}{1} \\
\hline position & $\delta_{\mathrm{C}}$ & $\delta_{\mathrm{H}}(\mathrm{JHz})$ & & $\delta_{\mathrm{C}}$ & $\delta_{\mathrm{H}}(\mathrm{JHz})$ \\
\hline 1 & $169.8 \mathrm{C}$ & & $1^{\prime}$ & $161.8 \mathrm{C}$ & \\
\hline 2 & $101.6 \mathrm{C}$ & & $2^{\prime}$ & $88.6 \mathrm{CH}$ & $5.56, \mathrm{~s}$ \\
\hline 3 & $159.9 \mathrm{C}$ & & $3^{\prime}$ & $170.1 \mathrm{C}$ & \\
\hline 4 & $105.4 \mathrm{CH}$ & $5.90, \mathrm{~s}$ & $4^{\prime}$ & $111.9 \mathrm{C}$ & \\
\hline 5 & $146.1 \mathrm{C}$ & & $5^{\prime}$ & $159.9 \mathrm{C}$ & \\
\hline 6 & $110.6 \mathrm{CH}$ & $6.42, \mathrm{~s}$ & $6^{\prime}$ & $72.3 \mathrm{CH}$ & 5.91, dd (overlap) \\
\hline 7 & $162.4 \mathrm{C}$ & & $7^{\prime}$ & $32.8 \mathrm{CH}_{2}$ & $1.94, \mathrm{~m} ; 2.08, \mathrm{~m}$ \\
\hline 8 & $134.6 \mathrm{C}$ & & 8' & $25.0 \mathrm{CH}_{2}$ & 1.47, m; 1.63, m \\
\hline 9 & $151.5 \mathrm{C}$ & & $9^{\prime}$ & $29.5 \mathrm{CH}_{2}$ & 1.17-1.36, m \\
\hline 10 & $106.9 \mathrm{CH}$ & $6.83, \mathrm{~d}(3.1)$ & $10^{\prime}$ & $31.4 \mathrm{CH}_{2}$ & 1.17-1.36, m \\
\hline 11 & $157.3 \mathrm{C}$ & & $11^{\prime}$ & $22.2 \mathrm{CH}_{2}$ & 1.17-1.36, m \\
\hline 12 & $106.7 \mathrm{CH}$ & $7.00, d(3.1)$ & $12^{\prime}$ & $13.4 \mathrm{CH}_{3}$ & $0.81, t(6.9)$ \\
\hline 13 & $125.6 \mathrm{C}$ & & $13^{\prime}$ & $52.6 \mathrm{CH}_{2}$ & $4.66, s$ \\
\hline 14 & $165.0 \mathrm{C}$ & & $14^{\prime}$ & $56.1 \mathrm{CH}_{3}$ & $3.93, s$ \\
\hline 15 & $51.6 \mathrm{CH}_{3}$ & $3.71, \mathrm{~s}$ & & & \\
\hline 16 & $55.2 \mathrm{CH}_{3}$ & $3.87, \mathrm{~s}$ & & & \\
\hline 17 & $21.1 \mathrm{CH}_{3}$ & $2.15, \mathrm{~s}$ & & & \\
\hline
\end{tabular}


Table S7. ${ }^{1} \mathrm{H}(500 \mathrm{MHz})$ and ${ }^{13} \mathrm{C}(125 \mathrm{MHz})$ NMR spectroscopic data for 2 in Acetone$d_{6}$.

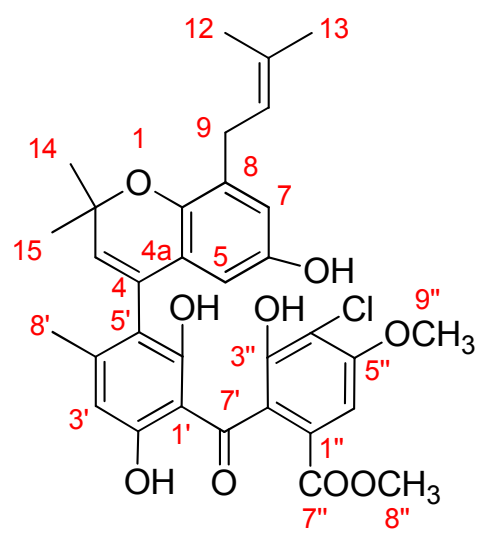

\begin{tabular}{llllll}
\hline & \multicolumn{5}{c}{$\mathbf{2}$} \\
\cline { 2 - 7 } position & $\delta_{\mathrm{C}}$ & $\delta_{\mathrm{H}}(\mathrm{JHz})$ & position & $\delta_{\mathrm{C}}$ & $\delta_{\mathrm{H}}(\mathrm{JHz})$ \\
\hline 1 & & & $1^{\prime}$ & $109.6 \mathrm{C}$ & \\
2 & $75.2 \mathrm{C}$ & & $2^{\prime}$ & $159.9 \mathrm{C}$ & \\
3 & $132.1 \mathrm{CH}$ & $5.58, \mathrm{~s}$ & $3^{\prime}$ & $107.9 \mathrm{CH}$ & $6.31, \mathrm{~s}$ \\
4 & $122.8 \mathrm{C}$ & & $4^{\prime}$ & $146.7 \mathrm{C}$ & \\
$4 \mathrm{a}$ & $128.9 \mathrm{C}$ & & $5^{\prime}$ & $117.0 \mathrm{C}$ & \\
5 & $108.6 \mathrm{CH}$ & $6.01, \mathrm{~d}(2.9)$ & $6^{\prime}$ & $160.5 \mathrm{C}$ & \\
6 & $150.6 \mathrm{C}$ & & $7^{\prime}$ & $198.7 \mathrm{C}$ & \\
7 & $115.8 \mathrm{CH}$ & $6.51, \mathrm{~d}(2.9)$ & $8^{\prime}$ & $19.7 \mathrm{CH}_{3}$ & $2.09, \mathrm{~s}$ \\
8 & $129.7 \mathrm{C}$ & & $1^{\prime \prime}$ & $128.1 \mathrm{C}$ & \\
$8 \mathrm{a}$ & $143.6 \mathrm{C}$ & & $2^{\prime \prime}$ & $126.5 \mathrm{C}$ & \\
9 & $28.1 \mathrm{CH}_{2}$ & $3.25, \mathrm{~d}(7.4)$ & $3^{\prime \prime}$ & $159.9 \mathrm{C}$ & \\
10 & $123.1 \mathrm{CH}^{\prime \prime}$ & $5.30, \mathrm{t}(7.5)$ & $4^{\prime \prime}$ & $113.8 \mathrm{C}$ & \\
11 & $131.1 \mathrm{C}$ & & $5^{\prime \prime}$ & $155.1 \mathrm{C}$ & \\
12 & $17.0 \mathrm{CH}_{3}$ & $1.74, \mathrm{~s}$ & $6^{\prime \prime}$ & $103.7 \mathrm{CH}$ & $7.17, \mathrm{~s}$ \\
13 & $25.0 \mathrm{CH}_{3}$ & $1.72, \mathrm{~s}$ & $7^{\prime \prime}$ & $165.5 \mathrm{C}$ & \\
14 & $26.6 \mathrm{CH}_{3}$ & $1.45, \mathrm{~s}$ & $8^{\prime \prime}$ & $51.7 \mathrm{CH}_{3}$ & $3.72, \mathrm{~s}$ \\
15 & $27.0 \mathrm{CH}_{3}$ & $1.48, \mathrm{~s}$ & $9^{\prime \prime}$ & $56.0 \mathrm{CH}_{3}$ & $3.97, \mathrm{~s}$ \\
$\mathrm{OH}-6$ & & $7.57, \mathrm{brs}$ & & & \\
\hline
\end{tabular}


Table S8. ${ }^{1} \mathrm{H}(500 \mathrm{MHz})$ and ${ }^{13} \mathrm{C}(125 \mathrm{MHz})$ NMR spectroscopic data for 3 in Acetone$d_{6}$.

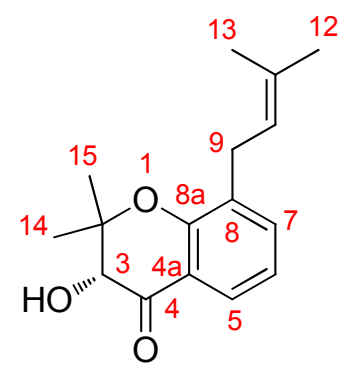

\begin{tabular}{lll}
\hline \multicolumn{3}{c}{$\mathbf{3}$} \\
\hline position & $\delta_{\mathrm{C}}$ & $\delta_{\mathrm{H}}(\mathrm{J} \mathrm{Hz})$ \\
\hline 1 & & \\
2 & $82.9 \mathrm{C}$ & \\
3 & $76.8 \mathrm{CH}$ & $4.41, \mathrm{~s}$ \\
4 & $193.9 \mathrm{C}$ & \\
$4 \mathrm{a}$ & $119.2 \mathrm{C}$ & \\
5 & $123.9 \mathrm{CH}$ & $7.61, \mathrm{dd}(7.8,1.7)$ \\
6 & $120.7 \mathrm{CH}$ & $6.98, \mathrm{t}(7.6)$ \\
7 & $136.0 \mathrm{CH}$ & $7.43, \mathrm{~d}(7.3)$ \\
8 & $131.1 \mathrm{C}$ & \\
$8 \mathrm{a}$ & $157.1 \mathrm{C}$ & \\
9 & $27.9 \mathrm{CH}_{2}$ & $3.37, \mathrm{dd}(7.6,15.3) ;$ \\
10 & $121.9 \mathrm{CH}^{2}$ & $5.28, \mathrm{dd}(7.5)$ \\
11 & $132.3 \mathrm{C}$ & \\
12 & $17.0 \mathrm{CH}_{3}$ & $1.74, \mathrm{~s}$ \\
13 & $25.0 \mathrm{CH}_{3}$ & $1.72, \mathrm{~s}$ \\
14 & $17.1 \mathrm{CH}_{3}$ & $1.20, \mathrm{~s}$ \\
15 & $26.0 \mathrm{CH}_{3}$ & $1.62, \mathrm{~s}$ \\
$\mathrm{OH}-3$ & & $4.63, \mathrm{brs}$ \\
\hline
\end{tabular}


Table S9. ${ }^{1} \mathrm{H}(500 \mathrm{MHz})$ and ${ }^{13} \mathrm{C}(125 \mathrm{MHz})$ NMR spectroscopic data for 4 in Acetone$d_{6}$.

\begin{tabular}{|c|c|c|}
\hline \multicolumn{3}{|c|}{4} \\
\hline position & $\delta_{\mathrm{C}}$ & $\delta_{\mathrm{H}}(\mathrm{JHz})$ \\
\hline 1 & & \\
\hline 2 & $162.9 \mathrm{C}$ & \\
\hline 3 & $87.1 \mathrm{CH}$ & $5.42, \mathrm{~s}$ \\
\hline 4 & $170.6 \mathrm{C}$ & \\
\hline 5 & $106.0 \mathrm{C}$ & \\
\hline 6 & $160.8 \mathrm{C}$ & \\
\hline 7 & $30.4 \mathrm{CH}_{2}$ & $2.52, \mathrm{t}(7.5)$ \\
\hline 8 & $27.1 \mathrm{CH}_{2}$ & $1.62, \mathrm{~m}$ \\
\hline 9 & $28.8 \mathrm{CH}_{2}$ & 1.29-1.35, m \\
\hline 10 & $28.8 \mathrm{CH}_{2}$ & 1.29-1.35, m \\
\hline 11 & $31.6 \mathrm{CH}_{2}$ & 1.29-1.35, m \\
\hline 12 & $22.4 \mathrm{CH}_{2}$ & 1.29-1.35, m \\
\hline 13 & $13.4 \mathrm{CH}_{3}$ & $0.88, \mathrm{t}(6.9)$ \\
\hline 14 & $8.5 \mathrm{CH}_{3}$ & $1.88, \mathrm{~s}$ \\
\hline 15 & $55.9 \mathrm{CH}_{3}$ & $3.88, \mathrm{~s}$ \\
\hline
\end{tabular}


Table S10. ${ }^{1} \mathrm{H}(500 \mathrm{MHz})$ and ${ }^{13} \mathrm{C}(125 \mathrm{MHz})$ NMR spectroscopic data for 6 in methanol- $d_{4}$.

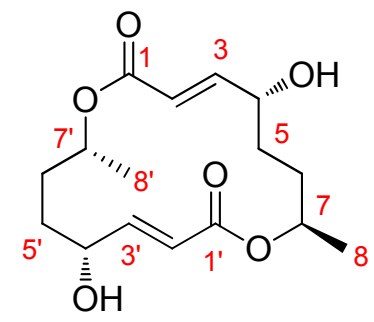

\begin{tabular}{lll}
\hline & \multicolumn{1}{c}{$\mathbf{6}$} \\
\cline { 2 - 3 } position & $\delta_{\mathrm{C}}$ & $\delta_{\mathrm{H}}(\mathrm{JHz})$ \\
\hline $1 / 1^{\prime}$ & $167.3 \mathrm{C}$ & \\
$2 / 2^{\prime}$ & $121.5 \mathrm{CH}$ & $6.99, \mathrm{dd}(3.6,15.8)$ \\
$3 / 3^{\prime}$ & $151.8 \mathrm{CH}$ & $5.95, \mathrm{dd}(1.9,15.8)$ \\
$4 / 4^{\prime}$ & $70.3 \mathrm{CH}$ & $4.41, \mathrm{~m}$ \\
$5 / 5^{\prime}$ & $31.4 \mathrm{CH}_{2}$ & $1.85, \mathrm{~m} ; 1.72, \mathrm{~m}$ \\
$6 / 6^{\prime}$ & $29.9 \mathrm{CH}_{2}$ & $1.72, \mathrm{~m} ; 1.60, \mathrm{~m}$ \\
$7 / 7^{\prime}$ & $71.4 \mathrm{CH}^{\prime}$ & $5.04, \mathrm{~m}$ \\
$8 / 8^{\prime}$ & $19.1 \mathrm{CH}_{3}$ & $1.24, \mathrm{~d}(6.4)$ \\
\hline
\end{tabular}


Table S11. ${ }^{1} \mathrm{H}(500 \mathrm{MHz})$ and ${ }^{13} \mathrm{C}(125 \mathrm{MHz})$ NMR spectroscopic data for 7 and 8 in methanol- $d_{4}$.
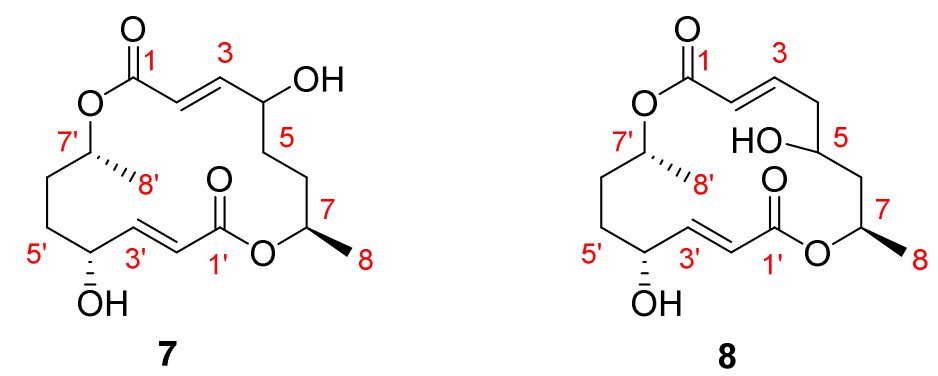

\begin{tabular}{|c|c|c|c|c|}
\hline \multirow[b]{2}{*}{ position } & \multicolumn{2}{|r|}{7} & \multicolumn{2}{|r|}{8} \\
\hline & $\delta_{\mathrm{C}}$ & $\delta_{\mathrm{H}}(\mathrm{JHz})$ & $\delta_{\mathrm{C}}$ & $\delta_{\mathrm{H}}(\mathrm{JHz})$ \\
\hline 1 & $166.0 \mathrm{C}$ & & $165.8 \mathrm{C}$ & \\
\hline 2 & $121.1 \mathrm{CH}$ & $5.81, \mathrm{dd}(1.4,15.8)$ & $123.6 \mathrm{CH}$ & $5.82, d(15.8)$ \\
\hline 3 & $149.6 \mathrm{CH}$ & $6.85, \mathrm{dd}(6.2,15.8)$ & $144.9 \mathrm{CH}$ & $6.94, \mathrm{dd}(7.5,15.8)$ \\
\hline 4 & $69.6 \mathrm{CH}$ & $4.05, \mathrm{~m}$ & $39.3 \mathrm{CH}_{2}$ & $2.54, \mathrm{~m} ; 2.30, \mathrm{~m}$ \\
\hline 5 & $29.0 \mathrm{CH}_{2}$ & $1.69, \mathrm{~m} ; 1.49, \mathrm{~m}$ & $67.0 \mathrm{CH}$ & $3.99, \mathrm{~m}$ \\
\hline 6 & $31.1 \mathrm{CH}_{2}$ & $1.69, \mathrm{~m}$ & $39.9 \mathrm{CH}_{2}$ & $1.86, \mathrm{~m}$ \\
\hline 7 & $70.1 \mathrm{CH}$ & $4.95, \mathrm{~m}$ & $68.0 \mathrm{CH}$ & $5.20, \mathrm{~m}$ \\
\hline 8 & $18.0, \mathrm{CH}_{3}$ & $1.14, d(6.3)$ & $18.5 \mathrm{CH}_{3}$ & $1.34, d(6.6)$ \\
\hline $1^{\prime}$ & $165.8 \mathrm{C}$ & & $165.5 \mathrm{C}$ & \\
\hline $2^{\prime}$ & $119.9 \mathrm{CH}$ & $5.84, \mathrm{dd}(2.0,15.9)$ & $120.2 \mathrm{CH}$ & $5.95, \mathrm{dd}(1.9,15.8)$ \\
\hline $3^{\prime}$ & $150.5 \mathrm{CH}$ & 6.89, dd $(3.6,15.9)$ & $150.6 \mathrm{CH}$ & $6.99, \mathrm{dd}(4.1,15.8)$ \\
\hline $4^{\prime}$ & $68.9 \mathrm{CH}$ & $4.33, \mathrm{~m}$ & $69.1 \mathrm{CH}$ & $4.45, \mathrm{~m}$ \\
\hline $5^{\prime}$ & $29.6 \mathrm{CH}_{2}$ & 1.69, m; 1.49., m & $29.7 \mathrm{CH}_{2}$ & $1.88, \mathrm{~m} ; 1.76, \mathrm{~m}$ \\
\hline $6^{\prime}$ & $28.0 \mathrm{CH}_{2}$ & $1.69, \mathrm{~m}$ & $27.9 \mathrm{CH}_{2}$ & $1.76, \mathrm{~m} ; 1.61, \mathrm{~m}$ \\
\hline $7^{\prime}$ & $70.3 \mathrm{CH}$ & $4.91, \mathrm{~m}$ & $70.2 \mathrm{CH}$ & $4.99, \mathrm{~m}$ \\
\hline $8^{\prime}$ & $17.7, \mathrm{CH}_{3}$ & $1.15, d(6.3)$ & $17.5 \mathrm{CH}_{3}$ & $1.25, d(6.4)$ \\
\hline
\end{tabular}


Table S12. ${ }^{1} \mathrm{H}(500 \mathrm{MHz})$ and ${ }^{13} \mathrm{C}(125 \mathrm{MHz})$ NMR spectroscopic data for 9 and $10 \mathrm{in}$ methanol- $d_{4}$.
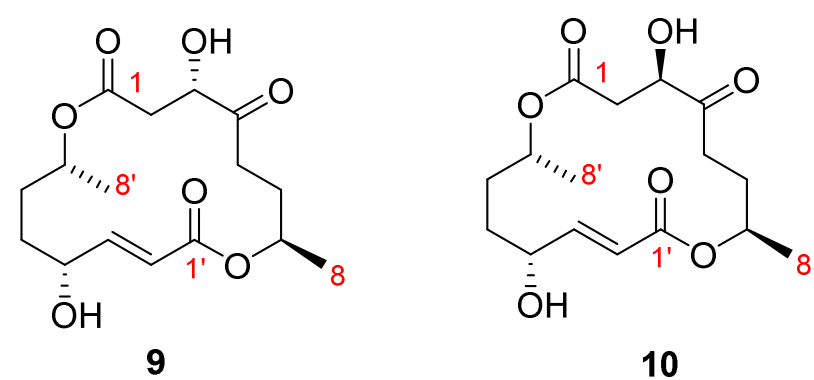

10

\begin{tabular}{|c|c|c|c|c|}
\hline \multirow[b]{2}{*}{ position } & \multicolumn{2}{|r|}{9} & \multicolumn{2}{|r|}{10} \\
\hline & $\delta_{\mathrm{C}}$ & $\delta_{\mathrm{H}}(\mathrm{JHz})$ & $\delta_{\mathrm{C}}$ & $\delta_{\mathrm{H}}(\mathrm{JHz})$ \\
\hline 7 & $170.1 \mathrm{C}$ & & $170.6 \mathrm{C}$ & \\
\hline 2 & $38.0 \mathrm{CH}_{2}$ & $\begin{array}{l}3.17 \text {, dd }(3.7,18) \\
2.79 \text {, dd }(4.3,18)\end{array}$ & $38.8 \mathrm{CH}_{2}$ & $\begin{array}{l}2.78 \text {, dd }(4.4,16.2) \\
2.68 \text {, dd }(3.9,16.2)\end{array}$ \\
\hline 3 & $72.3 \mathrm{CH}$ & $4.20, t(3.9)$ & $72.5 \mathrm{CH}$ & $4.38, \mathrm{dd}(3.9,4.4)$ \\
\hline 4 & 2 & & $211.1 \mathrm{C}$ & \\
\hline 5 & $32.0 \mathrm{CH}_{2}$ & $2.85, \mathrm{~m} ; 2.58, \mathrm{~m}$ & $34.1 \mathrm{CH}_{2}$ & $2.82, \mathrm{~m} ; 2.61, \mathrm{~m}$ \\
\hline 6 & $28.1 \mathrm{CH}_{2}$ & $2.03, \mathrm{~m} ; 1.83, \mathrm{~m}$ & $28.8 \mathrm{CH}_{2}$ & $1.93, \mathrm{~m}$ \\
\hline 7 & $70.0 \mathrm{CH}$ & $5.02, \mathrm{~m}$ & $70.7 \mathrm{CH}$ & $4.92, \mathrm{~m}$ \\
\hline 8 & $18.4 \mathrm{CH}_{3}$ & $1.30, d(6.3)$ & $19.1 \mathrm{CH}_{3}$ & $1.30, d(6.3)$ \\
\hline $1^{\prime}$ & $166.2 \mathrm{C}$ & & $166.2 \mathrm{C}$ & \\
\hline $2^{\prime}$ & $120.4 \mathrm{CH}$ & $5.92, \mathrm{dd}(1.7,15.9)$ & $120.4 \mathrm{CH}$ & $5.93, \mathrm{dd}(1.7,15.8)$ \\
\hline $3^{\prime}$ & $150.5 \mathrm{CH}$ & $6.80, \mathrm{dd}(4.9,15.9)$ & $150.6 \mathrm{CH}$ & $6.91, \mathrm{dd}(4.8,15.8)$ \\
\hline $4^{\prime}$ & $69.4 \mathrm{CH}$ & $4.41, \mathrm{~m}$ & $69.4 \mathrm{CH}$ & $4.43, \mathrm{~m}$ \\
\hline $5^{\prime}$ & $28.6 \mathrm{CH}_{2}$ & $1.83, \mathrm{~m} ; 1.68, \mathrm{~m}$ & $29.3 \mathrm{CH}_{2}$ & $1.85, \mathrm{~m}$ \\
\hline $6^{\prime}$ & $29.0 \mathrm{CH}_{2}$ & $1.83, \mathrm{~m} ; 1.48, \mathrm{~m}$ & $28.5 \mathrm{CH}_{2}$ & $1.70, \mathrm{~m} ; 1.57, \mathrm{~m}$ \\
\hline $7^{\prime}$ & $70.4 \mathrm{CH}$ & $4.88, \mathrm{~m}$ & $71.2 \mathrm{CH}$ & $4.86, \mathrm{~m}$ \\
\hline $8^{\prime}$ & $18.9 \mathrm{CH}_{3}$ & $1.17, d(6.3)$ & $17.6 \mathrm{CH}_{3}$ & $1.22, d(6.5)$ \\
\hline
\end{tabular}


Table S13. ${ }^{1} \mathrm{H}(500 \mathrm{MHz})$ and ${ }^{13} \mathrm{C}(125 \mathrm{MHz})$ NMR spectroscopic data for 11 in methanol- $d_{4}$.

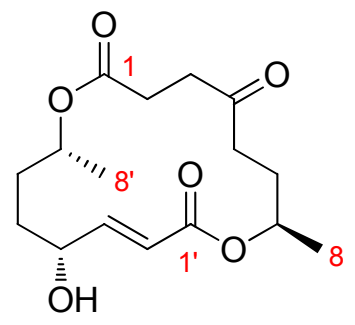

\begin{tabular}{lll}
\hline & \multicolumn{1}{c}{$\mathbf{1 1}$} \\
\cline { 2 - 3 } position & $\delta_{\mathrm{C}}$ & $\delta_{\mathrm{H}}(\mathrm{JHz})$ \\
\hline 1 & $172.8 \mathrm{C}$ & \\
2 & $27.9 \mathrm{CH}_{2}$ & $2.75, \mathrm{~m} ; 2.55, \mathrm{~m}$ \\
3 & $36.5 \mathrm{CH}_{2}$ & $2.87, \mathrm{~m} ; 2.42, \mathrm{~m}$ \\
4 & $209.0 \mathrm{C}$ & \\
5 & $38.2 \mathrm{CH}_{2}$ & $2.76, \mathrm{~m} ; 2.55, \mathrm{~m}$ \\
6 & $28.0 \mathrm{CH}_{2}$ & $2.07, \mathrm{~m} ; 1.82, \mathrm{~m}$ \\
7 & $70.6 \mathrm{CH}$ & $4.89, \mathrm{~m}$ overlap \\
8 & $19.1 \mathrm{CH}_{3}$ & $1.27, \mathrm{~d}(6.2)$ \\
$1^{\prime}$ & $166.2 \mathrm{C}$ & \\
$2^{\prime}$ & $120.2 \mathrm{CH}$ & $5.87, \mathrm{dd}(1.8,15.8)$ \\
$3^{\prime}$ & $151.1 \mathrm{CH}$ & $6.85, \mathrm{dd}(4.3,15.8)$ \\
$4^{\prime}$ & $69.0 \mathrm{CH}^{\prime}$ & $4.48, \mathrm{~m}$ \\
$5^{\prime}$ & $28.4 \mathrm{CH}_{2}$ & $1.78, \mathrm{~m}$ \\
$6^{\prime}$ & $28.2 \mathrm{CH}_{2}$ & $1.78, \mathrm{~m} ; 1.52, \mathrm{~m}$ \\
$7^{\prime}$ & $71.6 \mathrm{CH}^{\prime}$ & $4.89, \mathrm{~m}$ \\
$8^{\prime}$ & $17.8 \mathrm{CH}_{3}$ & $1.20, \mathrm{~d}(6.5)$ \\
\hline
\end{tabular}


Table S14. ${ }^{1} \mathrm{H}(500 \mathrm{MHz})$ and ${ }^{13} \mathrm{C}(125 \mathrm{MHz})$ NMR spectroscopic data for 12 in methanol- $d_{4}$.

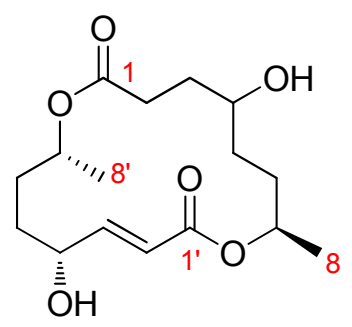

\begin{tabular}{lll}
\hline & \multicolumn{2}{c}{$\mathbf{1 2}$} \\
\cline { 2 - 3 } position & $\delta_{\mathrm{C}}$ & $\delta_{\mathrm{H}}(\mathrm{JHz})$ \\
\hline 1 & $173.5 \mathrm{C}$ & \\
2 & $29.7 \mathrm{CH}_{2}$ & $2.47, \mathrm{~m} ; 2.37, \mathrm{~m}$ \\
3 & $32.1 \mathrm{CH}_{2}$ & $1.92, \mathrm{~m} ; 1.51, \mathrm{~m}$ \\
4 & $69.5 \mathrm{CH}$ & $3.50, \mathrm{~m}$ \\
5 & $34.4 \mathrm{CH}_{2}$ & $1.51, \mathrm{~m}$ \\
6 & $30.7 \mathrm{CH}_{2}$ & $1.78, \mathrm{~m}$ \\
7 & $73.1 \mathrm{CH}$ & $4.92, \mathrm{~m}$ \\
8 & $19.6 \mathrm{CH}_{3}$ & $1.27, \mathrm{~d}(6.2)$ \\
$1^{\prime}$ & $166.0 \mathrm{C}$ & \\
$2^{\prime}$ & $120.3 \mathrm{CH}$ & $5.94, \mathrm{dd}(1.9,15.9)$ \\
$3^{\prime}$ & $150.8 \mathrm{CH}$ & $6.88, \mathrm{dd}(4.0,15.9)$ \\
$4^{\prime}$ & $69.0 \mathrm{CH}^{\prime}$ & $4.53, \mathrm{~m}$ \\
$5^{\prime}$ & $27.6 \mathrm{CH}_{2}$ & $1.78, \mathrm{~m}$ \\
$6^{\prime}$ & $27.4 \mathrm{CH}_{2}$ & $1.78, \mathrm{~m}$ \\
$7^{\prime}$ & $70.4 \mathrm{CH}^{\prime}$ & $4.92, \mathrm{~m}$ \\
$8^{\prime}$ & $17.2 \mathrm{CH}_{3}$ & $1.22, \mathrm{~d}(6.6)$ \\
\hline
\end{tabular}


Table S15. ${ }^{1} \mathrm{H}(500 \mathrm{MHz})$ and ${ }^{13} \mathrm{C}(125 \mathrm{MHz})$ NMR spectroscopic data for 13 and 14 in methanol- $d_{4}$.<smiles>[B]C(O)CC[C@H](O)/C=C/C(=O)O[C@H](C)CC[C@@H](O)/C=C/[13C](=O)O</smiles>

13<smiles>[B]C(O)CC[C@H](O)/C=C/[C@@H](O)CC[C@@H](C)OC(=O)/C=C/[C@@H](Br)OC</smiles>

14

\begin{tabular}{|c|c|c|c|c|}
\hline \multirow[b]{2}{*}{ position } & \multicolumn{2}{|r|}{13} & \multicolumn{2}{|r|}{14} \\
\hline & $\delta_{\mathrm{C}}$ & $\delta_{\mathrm{H}}(\mathrm{JHz})$ & $\delta_{\mathrm{C}}$ & $\delta_{\mathrm{H}}(\mathrm{JHz})$ \\
\hline 1 & $169.2 \mathrm{C}$ & & $167.2 \mathrm{C}$ & \\
\hline 2 & $119.7 \mathrm{CH}$ & $6.02, \mathrm{dd}(1.6,15.6)$ & $119.1 \mathrm{CH}$ & $6.05, \mathrm{dd}(1.6,15.6)$ \\
\hline 3 & $150.1 \mathrm{CH}$ & $6.89, \mathrm{dd}(5.1,15.6)$ & $151.0 \mathrm{CH}$ & $6.95, \mathrm{dd}(4.9,15.6)$ \\
\hline 4 & $70.0 \mathrm{CH}$ & $4.24, \mathrm{~m}$ & $69.9 \mathrm{CH}$ & 4.26, m, overlap \\
\hline 5 & $32.1 \mathrm{CH}$ & 1.48-1.77, m & $32.0 \mathrm{CH}_{2}$ & 1.72, m overlap \\
\hline 6 & $31.5 \mathrm{CH}_{2}$ & 1.48-1.77, m & $31.4 \mathrm{CH}_{2}$ & 1.72, m overlap \\
\hline 7 & $71.0 \mathrm{CH}$ & $4.98, m$ & $70.9 \mathrm{CH}$ & $4.98, m$ \\
\hline 8 & $18.8 \mathrm{CH}_{3}$ & $1.28, d(6.3)$ & $18.8 \mathrm{CH}_{3}$ & $1.27, d(6.3)$ \\
\hline $1^{\prime}$ & $166.5 \mathrm{C}$ & & $166.5 \mathrm{C}$ & \\
\hline $2^{\prime}$ & $120.6 \mathrm{C}$ & $6.00, \mathrm{dd}(1.7,15.7)$ & $119.7 \mathrm{CH}$ & $6.02, \mathrm{dd}(1.6,15.6)$ \\
\hline $3^{\prime}$ & $151.0 \mathrm{C}$ & $6.95, \mathrm{dd}(4.9,15.7)$ & $151.0 \mathrm{CH}$ & $6.95, \mathrm{dd}(4.9,15.6)$ \\
\hline $4^{\prime}$ & $70.3 \mathrm{CH}$ & $4.24, \mathrm{~m}$ & $70.3 \mathrm{CH}$ & 4.26, m, overlap \\
\hline $5^{\prime}$ & $32.5 \mathrm{CH}$ & 1.48-1.77, m & $32.5 \mathrm{CH}_{2}$ & 1.72, m overlap \\
\hline $6^{\prime}$ & $34.5 \mathrm{CH}_{2}$ & 1.48-1.77, m & $34.5 \mathrm{CH}_{2}$ & 1.55, m overlap \\
\hline $7^{\prime}$ & $67.2 \mathrm{CH}$ & $3.75, \mathrm{~m}$ & $67.2 \mathrm{CH}$ & 3.75, m overlap \\
\hline $8^{\prime}$ & $22.1 \mathrm{CH}_{3}$ & $1.18, d(6.2)$ & $22.1 \mathrm{CH}_{3}$ & $1.18, d(6.2)$ \\
\hline & & & $50.7 \mathrm{CH}_{3}$ & 3.75, m overlap \\
\hline
\end{tabular}


Table S16. ${ }^{1} \mathrm{H}(500 \mathrm{MHz})$ and ${ }^{13} \mathrm{C}(125 \mathrm{MHz})$ NMR spectroscopic data for 15 in methanol- $d_{4}$.

\begin{tabular}{|c|c|c|}
\hline \multirow[b]{2}{*}{ position } & \multicolumn{2}{|r|}{15} \\
\hline & $\delta_{\mathrm{C}}$ & $\delta_{\mathrm{H}}(\mathrm{JHz})$ \\
\hline 1 & $172.8 \mathrm{C}$ & \\
\hline 2 & $40.2 \mathrm{CH}_{2}$ & $\begin{array}{l}2.66, \mathrm{dd}(7.8,15.8) \\
2.58, \mathrm{dd}(5.5,15.8)\end{array}$ \\
\hline 3 & $67.8 \mathrm{CH}$ & $5.31, \mathrm{~m}$ \\
\hline 4 & $18.6 \mathrm{CH}_{3}$ & $1.33, d(6.3)$ \\
\hline $1^{\prime}$ & $166.1 \mathrm{C}$ & \\
\hline $2^{\prime}$ & $119.5 \mathrm{CH}$ & $6.00, \mathrm{dd}(1.6,15.6)$ \\
\hline 3 & $151.2 \mathrm{CH}$ & $6.95, \mathrm{dd}(4.9,15.6)$ \\
\hline $4^{\prime}$ & $70.3 \mathrm{CH}$ & $4.26, \mathrm{~m}$ \\
\hline $5^{\prime}$ & $32.5 \mathrm{CH}_{2}$ & $1.74, \mathrm{~m} ; 1.56, \mathrm{~m}$ \\
\hline $6^{\prime}$ & $34.5 \mathrm{CH}_{2}$ & $1.56, \mathrm{~m}$ \\
\hline $7^{\prime}$ & $67.2 \mathrm{CH}$ & $3.74, \mathrm{~m}$ \\
\hline 8' & $22.1 \mathrm{CH}_{3}$ & $1.18, d(6.2)$ \\
\hline
\end{tabular}


Table S17. ${ }^{1} \mathrm{H}(500 \mathrm{MHz})$ and ${ }^{13} \mathrm{C}(125 \mathrm{MHz})$ NMR spectroscopic data for 16 in methanol- $d_{4}$.

\begin{tabular}{|c|c|c|}
\hline \multirow[b]{2}{*}{ position } & \multicolumn{2}{|r|}{16} \\
\hline & $\delta_{\mathrm{C}}$ & $\delta_{\mathrm{H}}(\mathrm{JHz})$ \\
\hline 1 & $165.4 \mathrm{C}$ & \\
\hline 2 & $120.6 \mathrm{CH}$ & $5.90, d(15.7)$ \\
\hline 3 & $150.4 \mathrm{CH}$ & 7.04, m \\
\hline 4 & $31.8 \mathrm{CH}_{2}$ & $2.28, \mathrm{~m}$ \\
\hline 5 & $24.0 \mathrm{CH}_{2}$ & 1.62, m;1.53, m \\
\hline 6 & $38.1 \mathrm{CH}_{2}$ & $1.47, \mathrm{~m}$ \\
\hline 7 & $66.8 \mathrm{CH}$ & 3.74, m (overlap) \\
\hline 8 & $22.1 \mathrm{CH}_{3}$ & $1.18, d(6.2)$ \\
\hline $1^{\prime}$ & $101.7 \mathrm{CH}$ & 5.34 , brs \\
\hline $2^{\prime}$ & $68.4 \mathrm{CH}$ & 3.57 , brs \\
\hline $3^{\prime}$ & $74.1 \mathrm{CH}$ & $4.79, \mathrm{~s}$ \\
\hline $4^{\prime}$ & $68.9 \mathrm{CH}$ & $3.40, s$ \\
\hline $5^{\prime}$ & $76.3 \mathrm{CH}$ & $4.53, d(5.3)$ \\
\hline $6^{\prime}$ & $64.8 \mathrm{CH}_{2}$ & $\begin{array}{l}4.74, \mathrm{~d}(\mathrm{lov}) \text {, } \\
3.74 \text { ) }\end{array}$ \\
\hline
\end{tabular}


Table S18. ${ }^{1} \mathrm{H}$ and ${ }^{13} \mathrm{C}$ NMR spectroscopic data for $\mathbf{5}$ and 21.

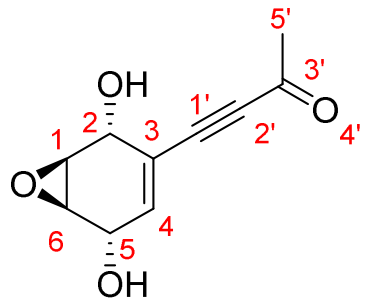

5

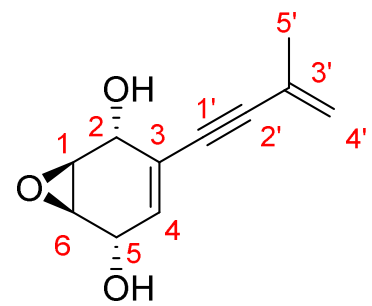

21

\begin{tabular}{|c|c|c|c|c|c|}
\hline \multirow[b]{2}{*}{ position } & \multicolumn{2}{|c|}{5 (methanol- $\left.d_{4}\right)$} & 5 (Acetone- $\left.d_{6}\right)$ & \multicolumn{2}{|c|}{$21^{\mathrm{a}}\left(\mathrm{DMSO}-d_{6}\right)^{[10,11]}$} \\
\hline & $\begin{array}{l}\delta_{\mathrm{C}} 125 \\
\mathrm{MHz}\end{array}$ & $\begin{array}{l}\delta_{\mathrm{H}}(\mathrm{J} \mathrm{Hz}) 500 \\
\mathrm{MHz}\end{array}$ & $\begin{array}{l}\delta_{\mathrm{H}}(\mathrm{J} \mathrm{Hz}) 600 \\
\mathrm{MHz}\end{array}$ & & \\
\hline 1 & $52.7 \mathrm{CH}$ & $3.35, \mathrm{~m}$ & $3.29, \mathrm{~m}$ & $53.5 \mathrm{CH}$ & $3.22, \mathrm{~m}$ \\
\hline $\begin{array}{l}2 \\
3\end{array}$ & $\begin{array}{l}63.8 \mathrm{CH} \\
119.9 \mathrm{C}\end{array}$ & $4.36, \mathrm{~m}$ & 4.36, brs & $\begin{array}{l}64.3 \mathrm{CH} \\
121.5 \mathrm{C}\end{array}$ & $4.15, \mathrm{~m}$ \\
\hline 4 & $137.3 \mathrm{CH}$ & $\begin{array}{l}6.30, \mathrm{dd} \\
(1.9,4.9)\end{array}$ & $6.26(1.3,4.0)$ & $133.1 \mathrm{CH}$ & $5.89, d(4.0)$ \\
\hline 5 & $61.6 \mathrm{CH}$ & $4.44, \mathrm{~m}$ & 4.43, brd (2.4) & $61.7, \mathrm{CH}$ & $4.28, \mathrm{~m}$ \\
\hline 6 & $51.6 \mathrm{CH}$ & $3.29, \mathrm{~m}$ & $3.23, \mathrm{~m}$ & $52.3 \mathrm{CH}$ & $3.17, \mathrm{~m}$ \\
\hline $1^{\prime}$ & 89.0 & & & $89.3 \mathrm{C}$ & \\
\hline $2^{\prime}$ & $87.1 \mathrm{C}$ & & & $90.3 \mathrm{C}$ & \\
\hline $3^{\prime}$ & $184.5 \mathrm{C}$ & & & $126.6 \mathrm{C}$ & \\
\hline $4^{\prime}$ & & & & $\begin{array}{l}123.0 \\
\mathrm{CH}_{2}\end{array}$ & $\begin{array}{l}5.34, \mathrm{~m} ; 5.29, \\
\mathrm{~m}\end{array}$ \\
\hline $\begin{array}{l}5^{\prime} \\
\mathrm{OH}-2 \\
\mathrm{OH}-5\end{array}$ & $31.3 \mathrm{CH}_{3}$ & $2.38, \mathrm{~s}$ & $2.32, \mathrm{~s}$ & $23.6 \mathrm{CH}_{3}$ & $\begin{array}{l}1.88, \mathrm{~s} \\
5.49, \mathrm{~d}(8.2) \\
5.25, \mathrm{~d}(7.5)\end{array}$ \\
\hline
\end{tabular}

\footnotetext{
${ }^{\mathrm{a}}$ The NMR data were reassigned based on the 1D, 2D NMR spectra.
} 
Table S19. ${ }^{1} \mathrm{H}(500 \mathrm{MHz})$ and ${ }^{13} \mathrm{C}(125 \mathrm{MHz})$ NMR spectroscopic data for $\mathbf{H 3}$ in methanol- $d_{4}$

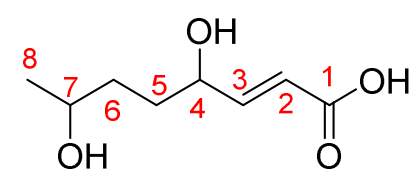

H3

\begin{tabular}{lll}
\hline & \multicolumn{2}{c}{$\mathrm{H} 3$} \\
\cline { 2 - 3 } position & $\delta_{\mathrm{C}}$ & $\delta_{\mathrm{H}}(\mathrm{JHz})$ \\
\hline 1 & $171.0 \mathrm{C}$ & \\
2 & $122.3 \mathrm{CH}$ & $6.00, \mathrm{dd}(1.2,15.7)$ \\
3 & $151.4 \mathrm{CH}$ & $6.90, \mathrm{dd}(5.1,15.7)$ \\
4 & $68.7 \mathrm{CH}$ & $4.25, \mathrm{~m}$ \\
$5^{\mathrm{a}}$ & $35.9 \mathrm{CH}_{2}$ & $1.58,1.74, \mathrm{~m}$ \\
$6^{\mathrm{a}}$ & $34.0 \mathrm{CH}_{2}$ & $1.50,1.74, \mathrm{~m}$ \\
7 & $71.8 \mathrm{CH}$ & $3.75, \mathrm{~m}$ \\
8 & $23.5 \mathrm{CH}_{3}$ & $1.18, \mathrm{~d}(6.2)$ \\
\hline a The data were exchangeable
\end{tabular}


Table S20. ${ }^{1} \mathrm{H}(500 \mathrm{MHz})$ and ${ }^{13} \mathrm{C}(125 \mathrm{MHz})$ NMR spectroscopic data for $\mathbf{H 4}$ in methanol- $d_{4}$.

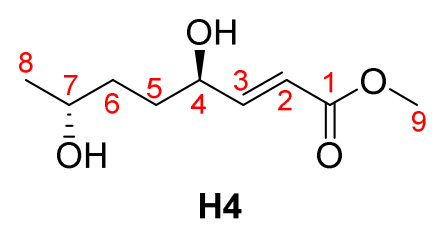

\begin{tabular}{lll}
\hline & \multicolumn{2}{c}{$\mathbf{H} \mathbf{4}$} \\
\cline { 2 - 3 } position & $\delta_{\mathrm{C}}$ & $\delta_{\mathrm{H}}(\mathrm{JHz})$ \\
\hline 1 & $168.7 \mathrm{C}$ & \\
2 & $120.4 \mathrm{CH}$ & $6.03, \mathrm{dd}(1.7,15.8)$ \\
3 & $152.7 \mathrm{CH}$ & $6.96, \mathrm{dd}(4.9,15.7)$ \\
4 & $68.5 \mathrm{CH}$ & $4.25, \mathrm{~m}$ \\
$5^{\mathrm{a}}$ & $35.9 \mathrm{CH}_{2}$ & $1.58,1.73, \mathrm{~m}$ \\
$6^{\mathrm{a}}$ & $33.9 \mathrm{CH}_{2}$ & $1.50,1.73, \mathrm{~m}$ \\
7 & $71.6 \mathrm{CH}$ & $3.73, \mathrm{~m}$ \\
8 & $23.5 \mathrm{CH}_{3}$ & $1.17, \mathrm{~d}(6.2)$ \\
9 & $52.0 \mathrm{OCH}_{3}$ & $3.73, \mathrm{~s}$ \\
\hline & \multicolumn{2}{l}{ a } \\
\multicolumn{3}{l}{ The data were exchangeable }
\end{tabular}


Table S21. ${ }^{13} \mathrm{C}$ NMR analysis of ficioilde A (6) after feeding with $\left[1-{ }^{13} \mathrm{C}\right]$ sodium acetate, $\left[2-{ }^{13} \mathrm{C}\right]$ sodium acetate, and $\left[1,2-{ }^{13} \mathrm{C}_{2}\right]$ sodium acetate
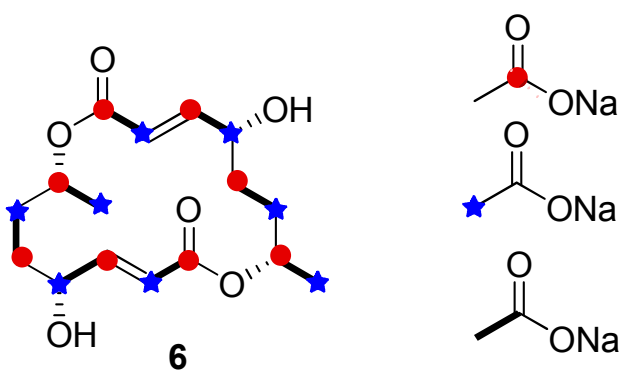

\begin{tabular}{|c|c|c|c|c|}
\hline \multirow{3}{*}{ position } & \multicolumn{4}{|c|}{ Ficiolide A } \\
\hline & $\delta_{\mathrm{C}}$ & Enrich & & $J_{c c}^{c}(H z)$ \\
\hline & \multicolumn{4}{|c|}{$\left[1-{ }^{13} \mathrm{C}\right] \mathrm{AcONa} \quad\left[2-{ }^{13} \mathrm{C}\right] \mathrm{AcONa}$} \\
\hline $1 / 1^{\prime}$ & $167.3 \mathrm{C}$ & $3.42^{b}$ & 1.05 & 148.5 \\
\hline $2 / 2^{\prime}$ & $121.5 \mathrm{CH}$ & 1.00 & 2.87 & 148.5 \\
\hline 3/3' & $151.8 \mathrm{CH}$ & 3.47 & 1.00 & 89.83 \\
\hline $4 / 4^{\prime}$ & $70.3 \mathrm{CH}$ & 1.38 & 2.84 & 89.85 \\
\hline $5 / 5^{\prime}$ & $31.4 \mathrm{CH}_{2}$ & 3.04 & 0.95 & 71.05 \\
\hline $6 / 6^{\prime}$ & $29.9 \mathrm{CH}_{2}$ & 0.89 & 2.94 & 71.05 \\
\hline $7 / 7^{\prime}$ & $71.4 \mathrm{CH}$ & 3.34 & 0.97 & 77.95 \\
\hline $8 / 8^{\prime}$ & $19.1 \mathrm{CH}_{3}$ & 1.11 & 3.20 & 78.05 \\
\hline
\end{tabular}

${ }^{a}{ }^{13} \mathrm{C}$ signal intensity of each peak in the labeled ficiolide $\mathrm{A}$ divided by that of the corresponding signal in the unlabeled, normalized to give an enrichment ratio of ficiolide A for unriched peak (C-2/2' for $\left[1-{ }^{13} \mathrm{C}\right]$ sodium acetate labeling and $\mathrm{C}-3 / 3$ ' for $\left[2-{ }^{13} \mathrm{C}\right]$ sodium acetate labeling).

${ }^{b}$ The numbers in bold type indicate ${ }^{13} \mathrm{C}$-enriched atoms from ${ }^{13} \mathrm{C}$-labeled acetate.

${ }^{c}$ Detected with the ${ }^{13} \mathrm{C}$-enriched sample from the feeding experiment with $\left[1,2-{ }^{13} \mathrm{C}_{2}\right]$ sodium acetate. Coupled carbon atoms are in parentheses. 
Table S22. HR-ESI-MS data of new compounds

\begin{tabular}{|c|c|c|c|c|}
\hline compound & 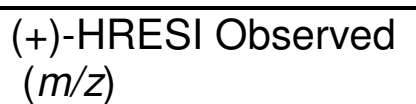 & Ion & Calculated Formula & $\begin{array}{l}\text { Ion Calculated } \\
(\mathrm{m} / \mathrm{z})\end{array}$ \\
\hline 1 & 601.2288 & {$[\mathrm{M}+\mathrm{H}]^{+}$} & $\mathrm{C}_{31} \mathrm{H}_{37} \mathrm{O}_{12}$ & 601.2207 \\
\hline 2 & 609.1893 & {$[\mathrm{M}+\mathrm{H}]^{+}$} & $\mathrm{C}_{33} \mathrm{H}_{34} \mathrm{O}_{9} \mathrm{Cl}$ & 609.1886 \\
\hline 3 & 261.1489 & {$[\mathrm{M}+\mathrm{H}]^{+}$} & $\mathrm{C}_{16} \mathrm{H}_{21} \mathrm{O}_{3}$ & 261.1485 \\
\hline 4 & 239.1639 & {$[\mathrm{M}+\mathrm{H}]^{+}$} & $\mathrm{C}_{14} \mathrm{H}_{23} \mathrm{O}_{3}$ & 239.1638 \\
\hline 5 & 217.0474 & {$[\mathrm{M}+\mathrm{Na}]^{+}$} & $\mathrm{C}_{10} \mathrm{H}_{10} \mathrm{O}_{4} \mathrm{Na}$ & 217.0471 \\
\hline 7 & 313.1640 & {$[\mathrm{M}+\mathrm{H}]^{+}$} & $\mathrm{C}_{16} \mathrm{H}_{25} \mathrm{O}_{6}$ & 313.1646 \\
\hline 8 & 313.1647 & {$[\mathrm{M}+\mathrm{H}]^{+}$} & $\mathrm{C}_{16} \mathrm{H}_{25} \mathrm{O}_{6}$ & 313.1646 \\
\hline 9 & 346.1862 & {$[\mathrm{M}+\mathrm{NH}]^{+}$} & $\mathrm{C}_{16} \mathrm{H}_{25} \mathrm{O}_{6}$ & 313.1646 \\
\hline 10 & 351.1415 & {$[\mathrm{M}+\mathrm{Na}]^{+}$} & $\mathrm{C}_{16} \mathrm{H}_{24} \mathrm{O}_{7} \mathrm{Na}$ & 351.1414 \\
\hline 11 & 313.1651 & {$[\mathrm{M}+\mathrm{H}]^{+}$} & $\mathrm{C}_{16} \mathrm{H}_{25} \mathrm{O}_{6}$ & 313.1646 \\
\hline 12 & 315.1801 & {$[\mathrm{M}+\mathrm{H}]^{+}$} & $\mathrm{C}_{16} \mathrm{H}_{27} \mathrm{O}_{6}$ & 315.1802 \\
\hline 13 & 331.1754 & {$[\mathrm{M}+\mathrm{H}]^{+}$} & $\mathrm{C}_{16} \mathrm{H}_{27} \mathrm{O}_{7}$ & 331.1751 \\
\hline 14 & 367.1731 & {$[\mathrm{M}+\mathrm{Na}]^{+}$} & $\mathrm{C}_{16} \mathrm{H}_{28} \mathrm{O}_{7} \mathrm{Na}$ & 367.1727 \\
\hline 15 & 283.1152 & {$[\mathrm{M}+\mathrm{H}]^{+}$} & $\mathrm{C}_{12} \mathrm{H}_{21} \mathrm{O}_{6}$ & 283.1152 \\
\hline 16 & 303.1440 & {$[\mathrm{M}+\mathrm{H}]^{+}$} & $\mathrm{C}_{14} \mathrm{H}_{23} \mathrm{O}_{7}$ & 303.1438 \\
\hline H3 & 197.0786 & {$[\mathrm{M}+\mathrm{Na}]^{+}$} & $\mathrm{C}_{8} \mathrm{H}_{14} \mathrm{O}_{4} \mathrm{Na}$ & 197.0784 \\
\hline $\mathrm{H} 4$ & 211.0938 & {$[\mathrm{M}+\mathrm{Na}]^{+}$} & $\mathrm{C}_{9} \mathrm{H}_{16} \mathrm{O}_{4} \mathrm{Na}$ & 211.0941 \\
\hline
\end{tabular}




\section{Supplementary Figures}

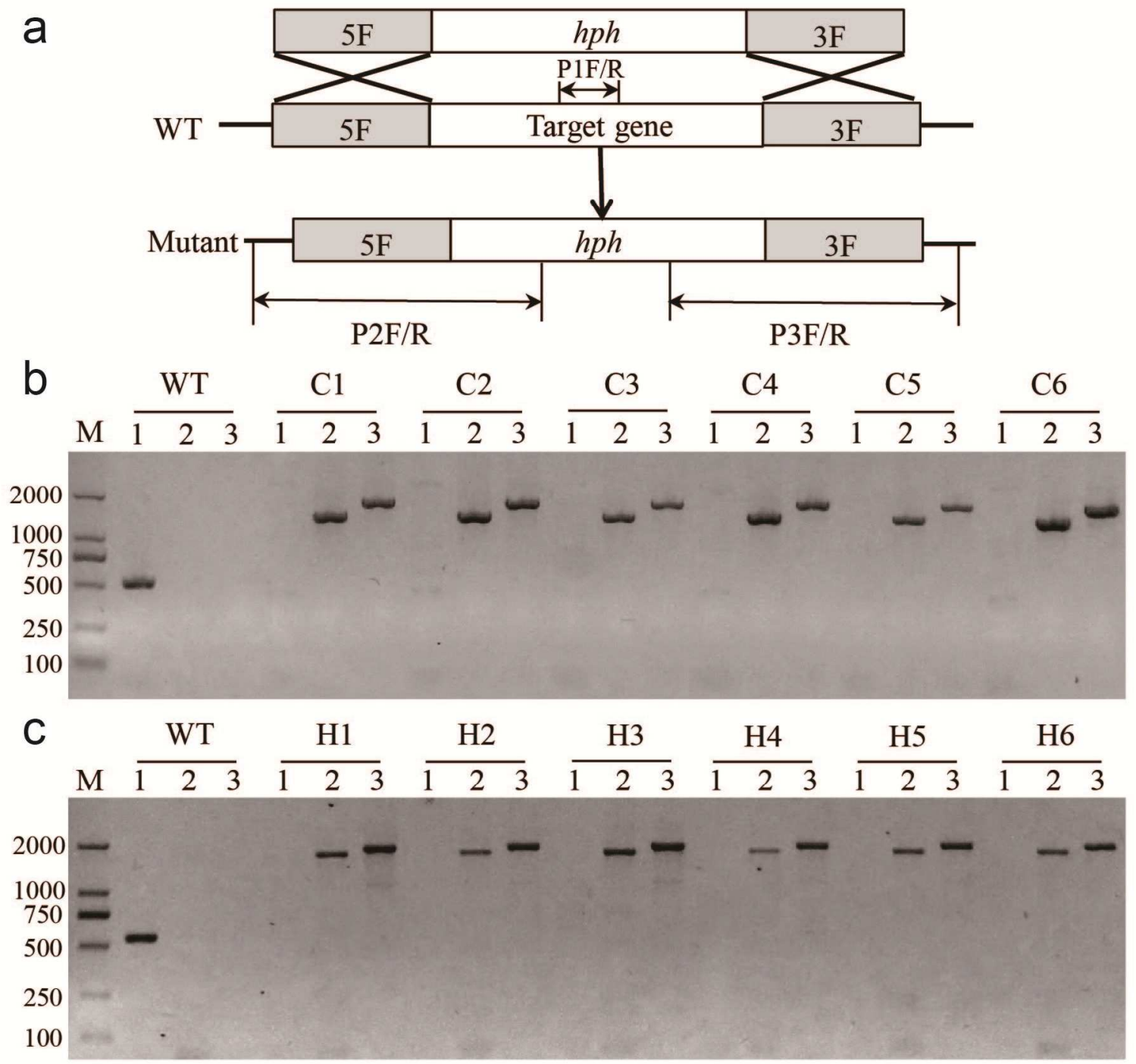

Figure S1 Generation of PfcclA and PfhdaA deletion strains. (a) Schematic illustration for disruption of targeted genes. The hph gene in vector pDHt-hyg is used as a selection maker after transformation. Transformation was performed by homologous recombination via either ATMA or protoplast transformation methods. Three pairs of primers including P1F/R, P2F/R and P3F/R were used for transformant screening. (b) Confirmation of $\triangle$ PfcclA strains by diagnostic PCR, specific bands (ca. 1.5-2 kb) were only found in mutants but not in WT. (c) Confirmation of $\triangle$ PfhdaA strains by diagnostic PCR, specific bands (ca. $1.5-2 \mathrm{~kb}$ ) were only detected in mutants but not in WT. 

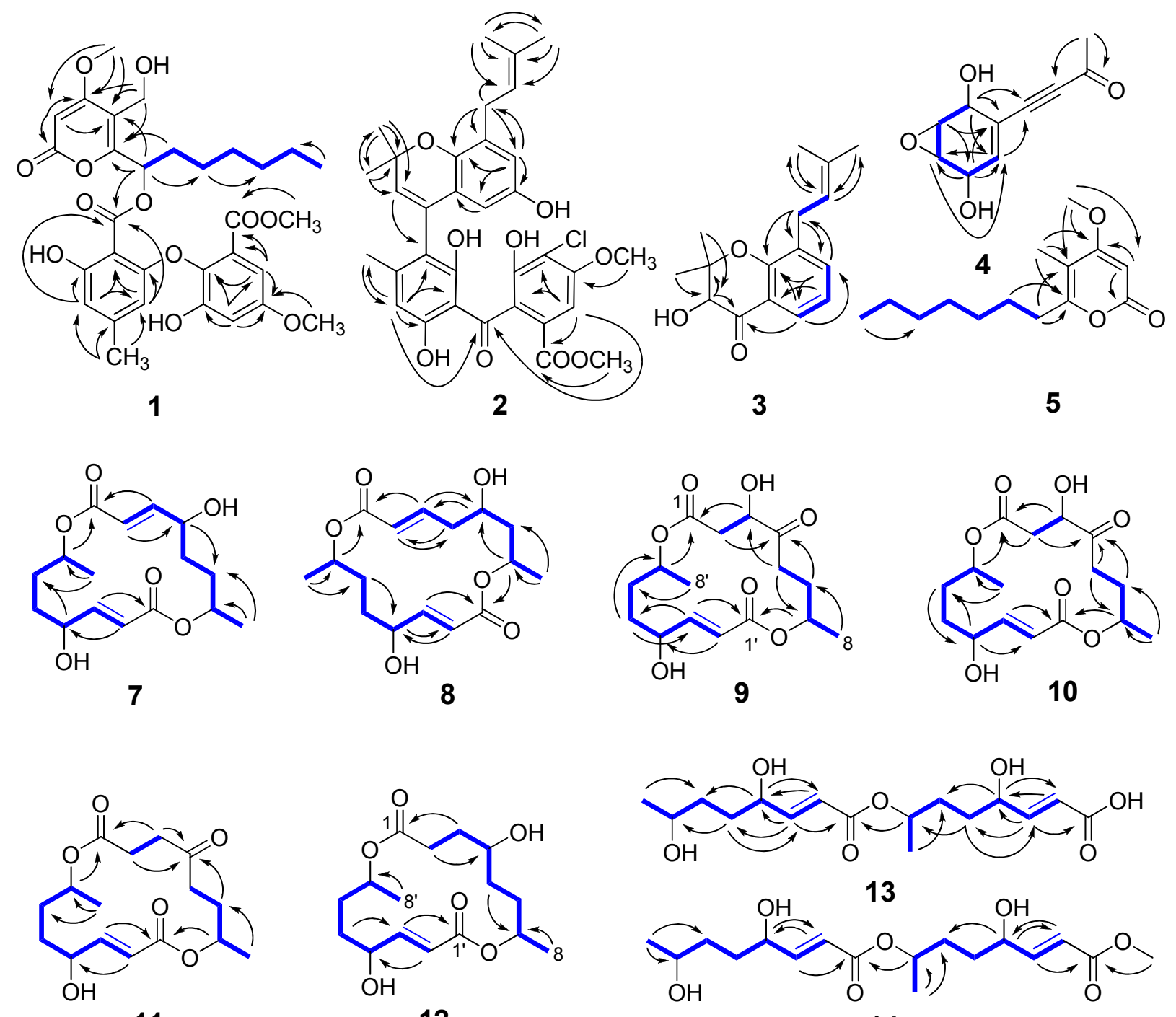

11

12
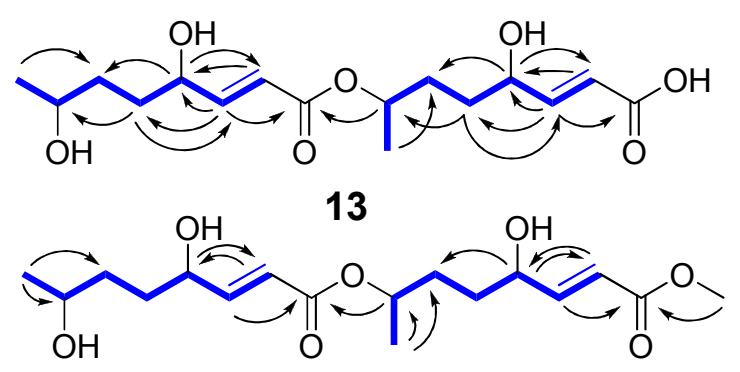

14

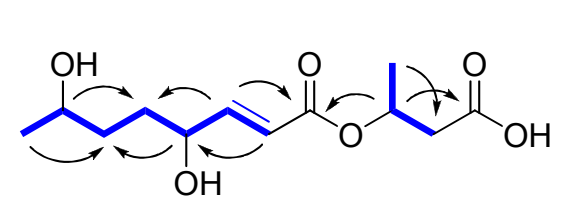

15

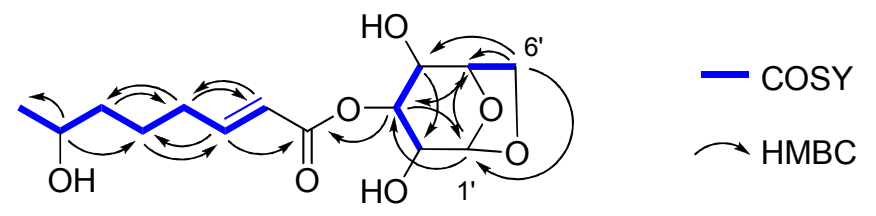

16

Figure S2 Key HMBC (arrow) and $1 \mathrm{H}-1 \mathrm{H}$ COSY (blue boldline) correlations of 1-5 and 7-16. 


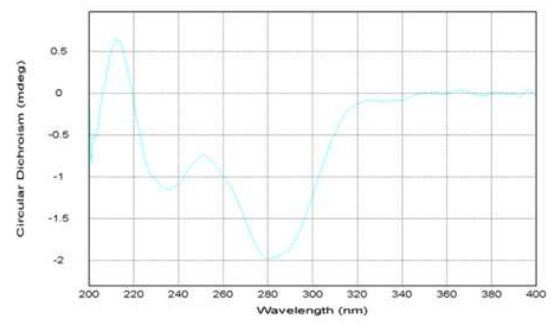

H1

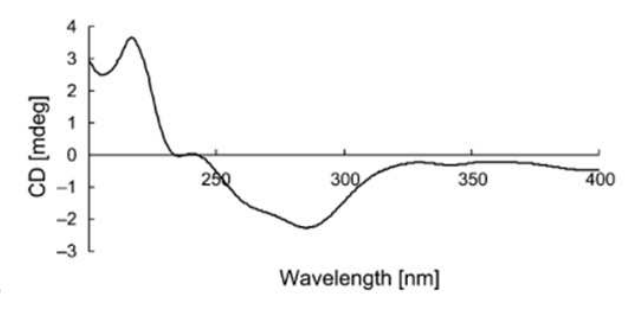

Ficipyrone A

Figure S3. The experiment ECD spectrum of $\mathbf{H 1}$ and previous reported ECD spectrum of ficipyrone $\mathrm{A}$ 

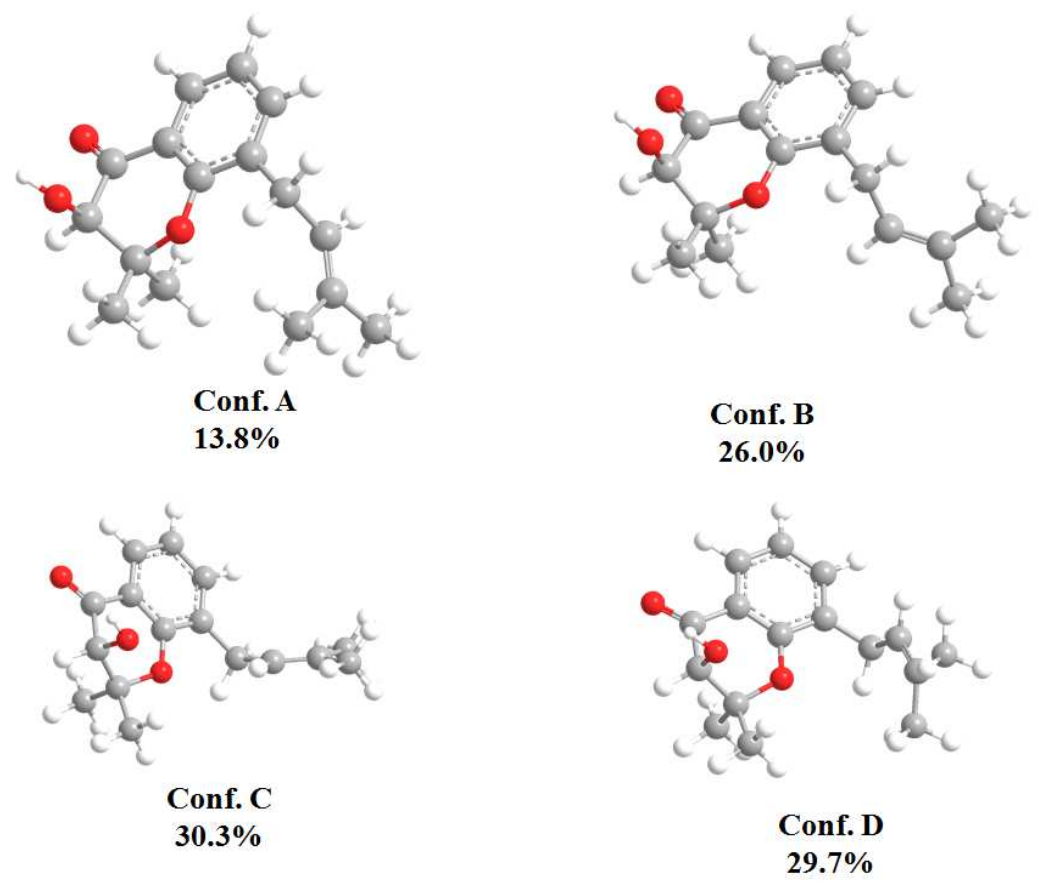

Figure S4. DFT-optimized structures for low-energy conformers of $(3 R)-3$ at B3LYP/6-31+g(d) level in methanol (PCM) (Conformer populations were calculated using the Gibbs free energy and Boltzmann population at $298 \mathrm{~K}$ estimated thereof). 


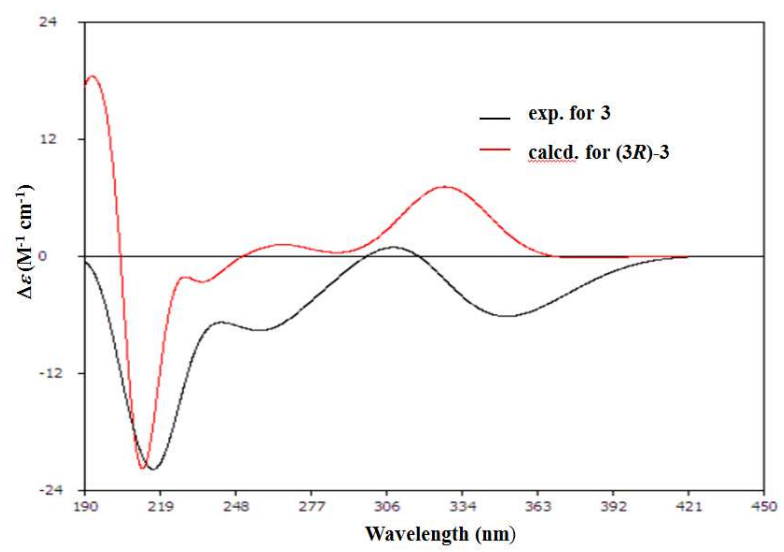

Figure S5. Experimental ECD and calculated spectra of 3. 


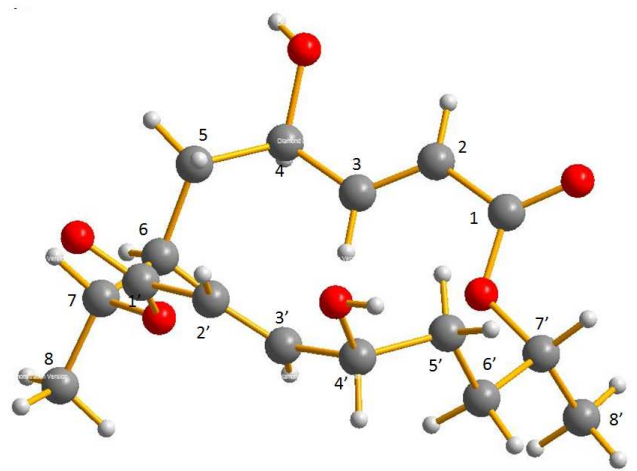

Figure S6. Ortep diagram for single crystal X-ray geometry of $\mathbf{6}$. 


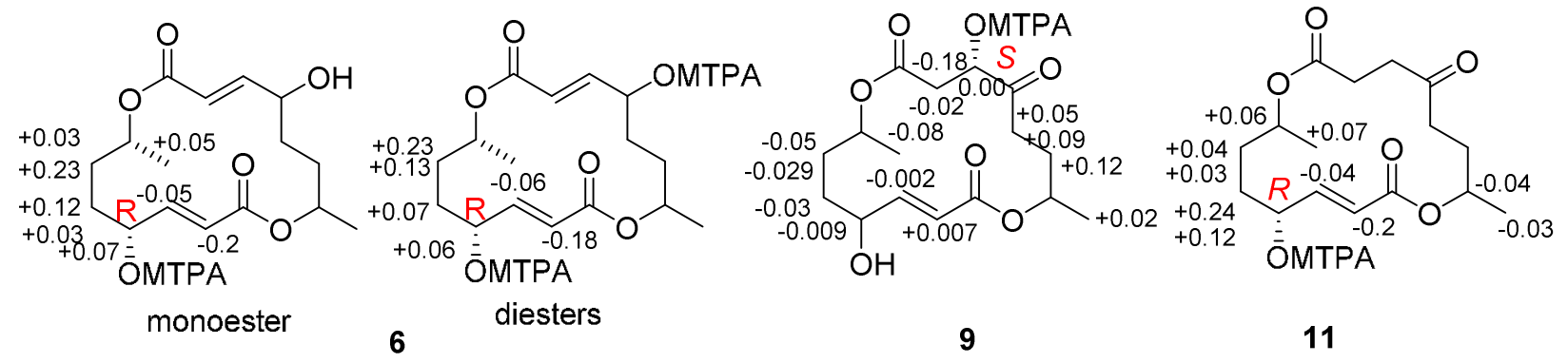

Figure S7. $\Delta \delta_{S_{-} R}$ values for the MTPA ester derivatives of $\mathbf{6 , 9}$ and 11. 
<smiles>CC(=O)C#CC1=C[C@@H](O)C2O[C@H]2[C@H]1O</smiles>

5

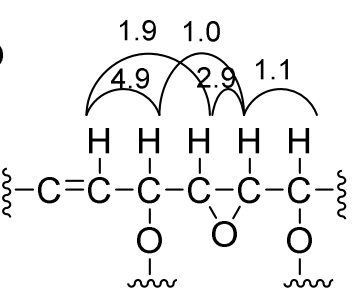

copuling constants

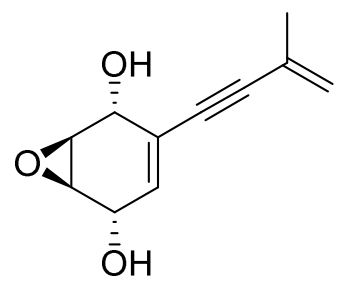

21

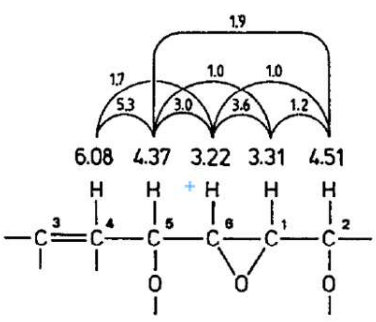

copuling constants

Figure S8. The relative configuration of $\mathbf{5}$ determined by comparison of coupling constants of 5 and 21. 


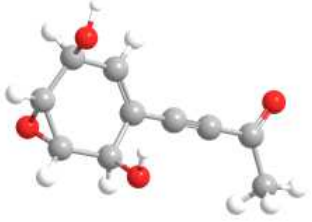

Conf. A

$31.8 \%$

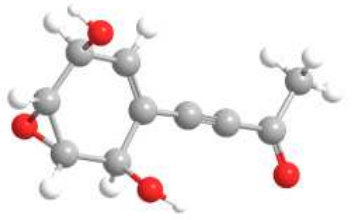

Conf. C

$17.9 \%$

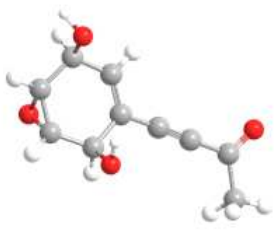

Conf. B

$27.3 \%$

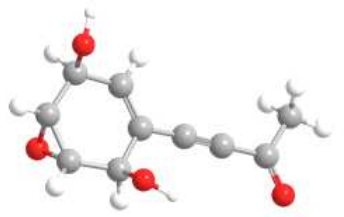

Conf. D

Figure S9. DFT-optimized structures for low-energy conformers of $(1 S, 2 R, 5 S, 6 R)-5$ at $B 3 L Y P / 6-31+g(d)$ level in methanol (PCM) (Conformer populations were calculated using the Gibbs free energy and Boltzmann population at $298 \mathrm{~K}$ estimated thereof). 


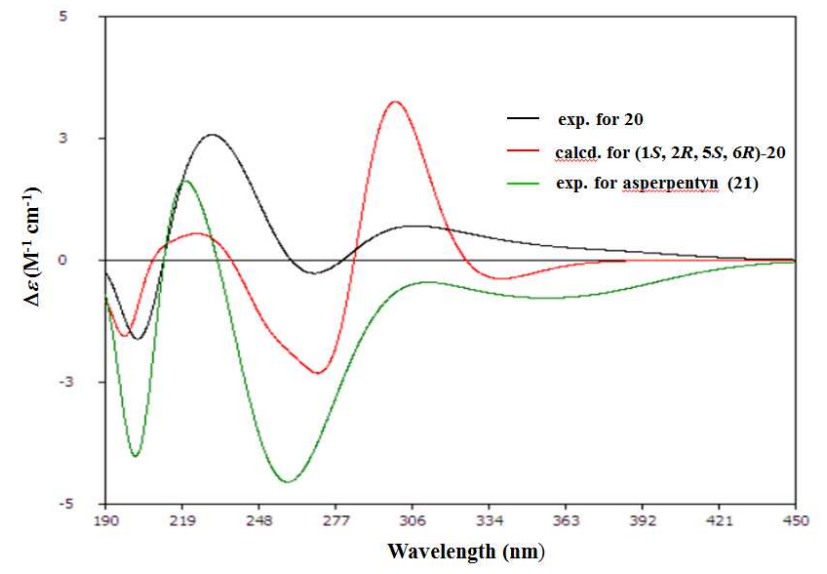

Figure S10. Experimental ECD and calculated spectra of $\mathbf{5}$ and 21. 


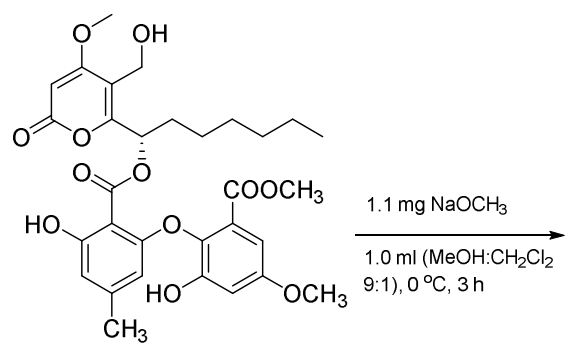

$10.8 \mathrm{mg}$

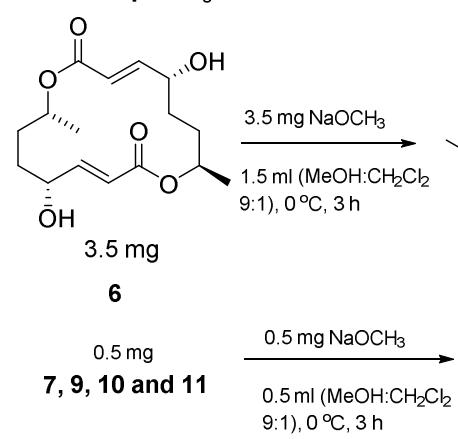

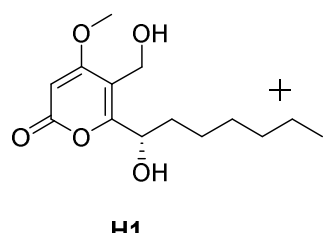

H1

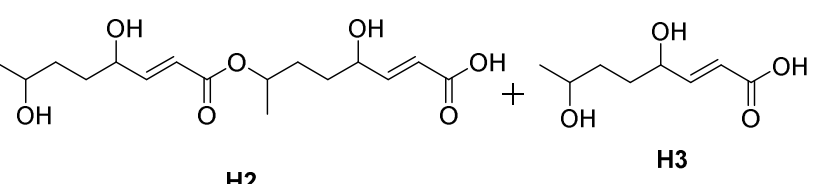

H2

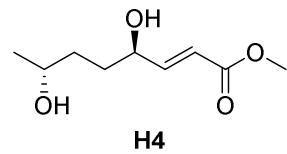

H4

Figure S11. Schematic illustration for hydrolysis of 1, 6, 7, 9-11. 

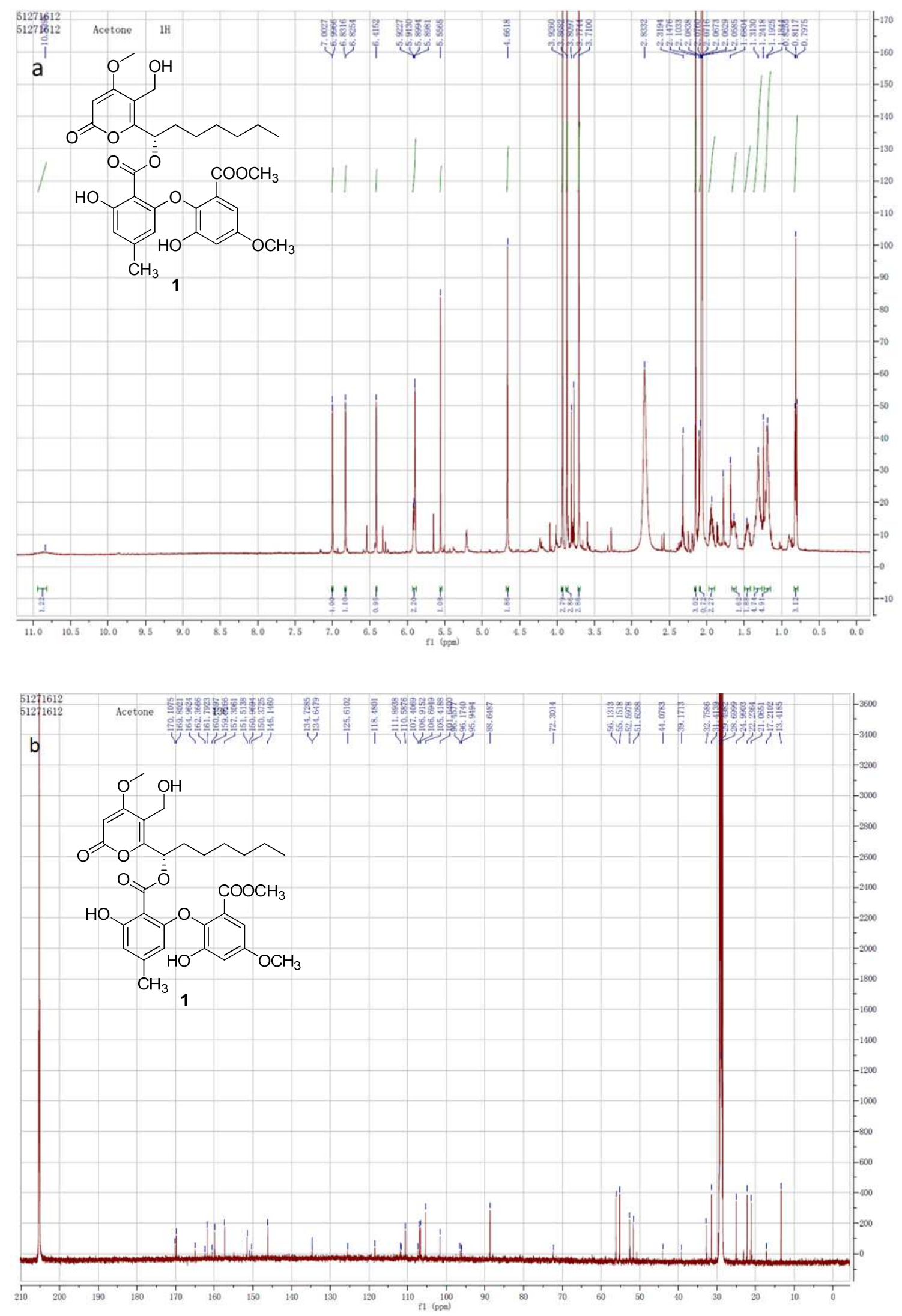


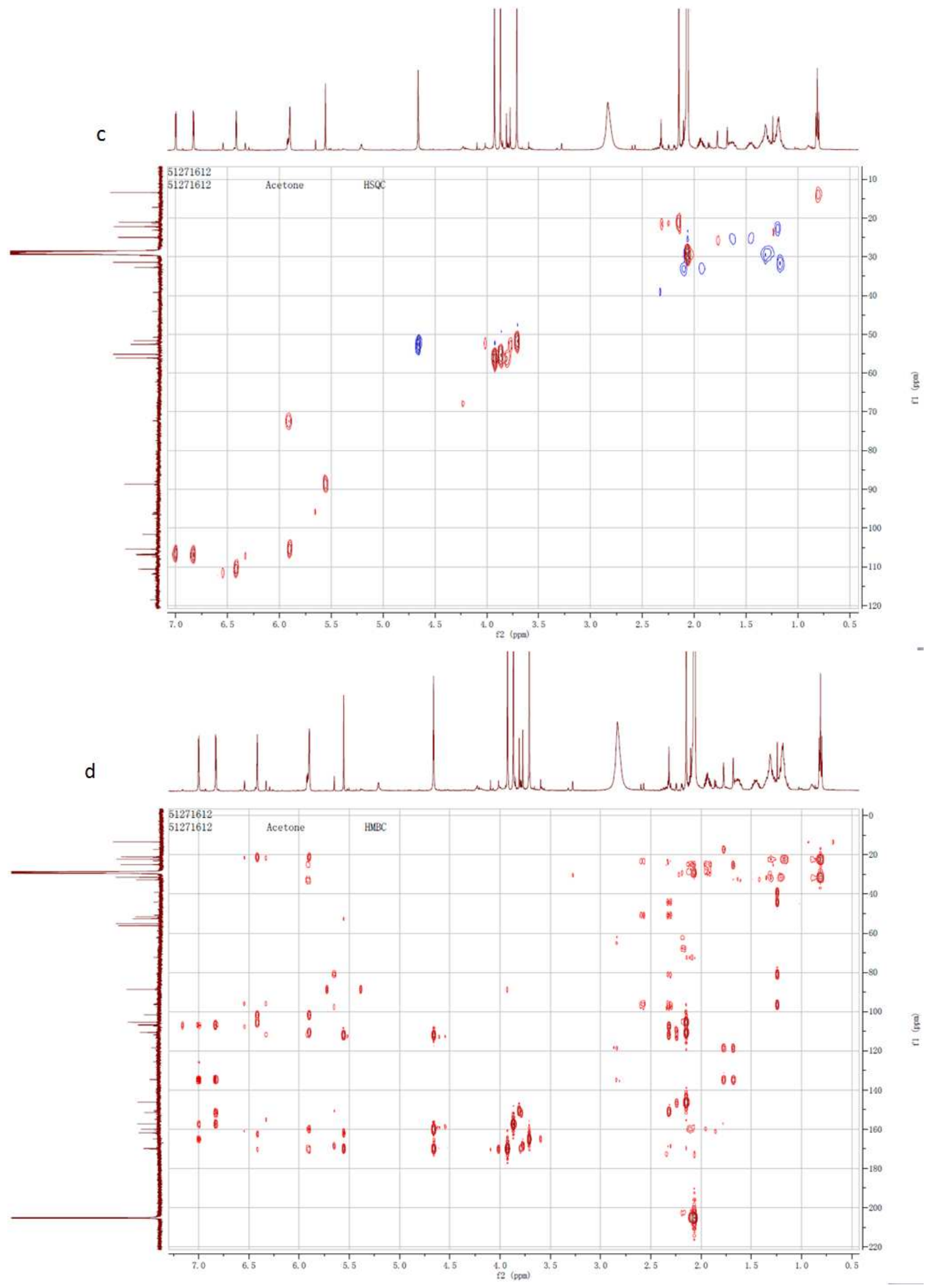




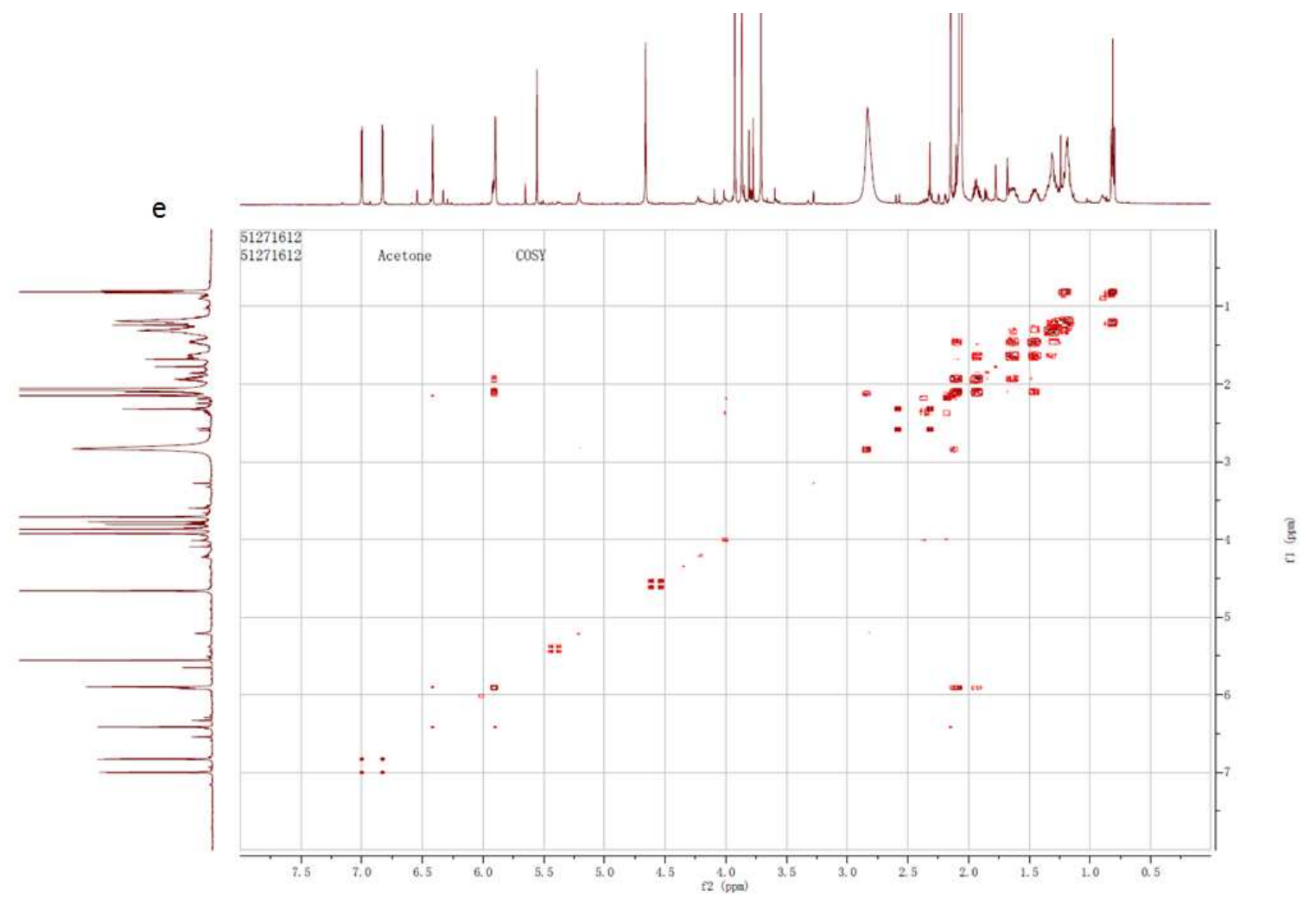

Figure S12. NMR analysis of 1 in acetone- $d_{6}$. (a) ${ }^{1} \mathrm{H}$ NMR spectrum. (b) ${ }^{13} \mathrm{C}$ NMR spectrum. (c) HSQC spectrum. (d) HMBC spectrum. (e) ${ }^{1} \mathrm{H}-{ }^{1} \mathrm{H}$ COSY spectrum. 


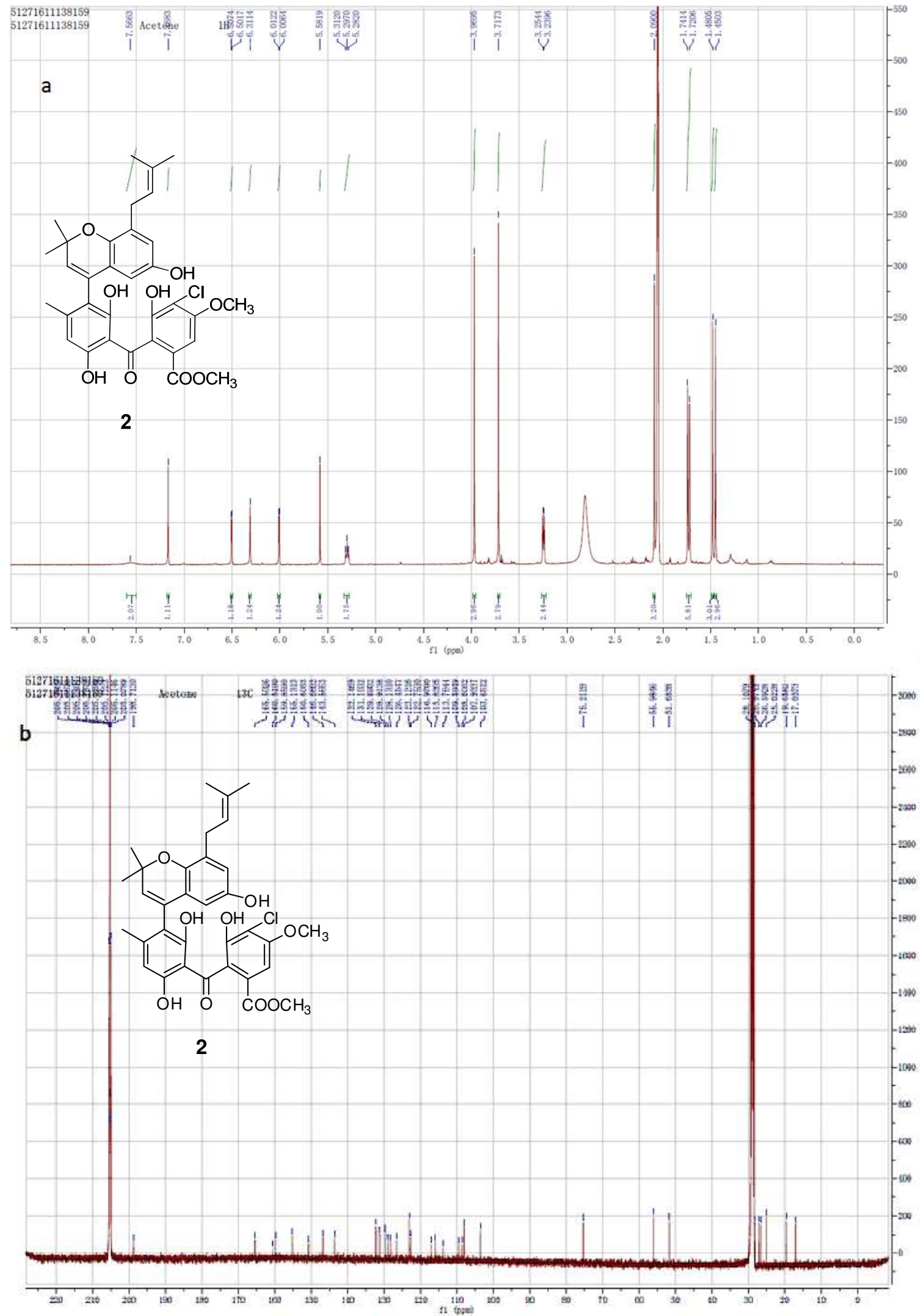



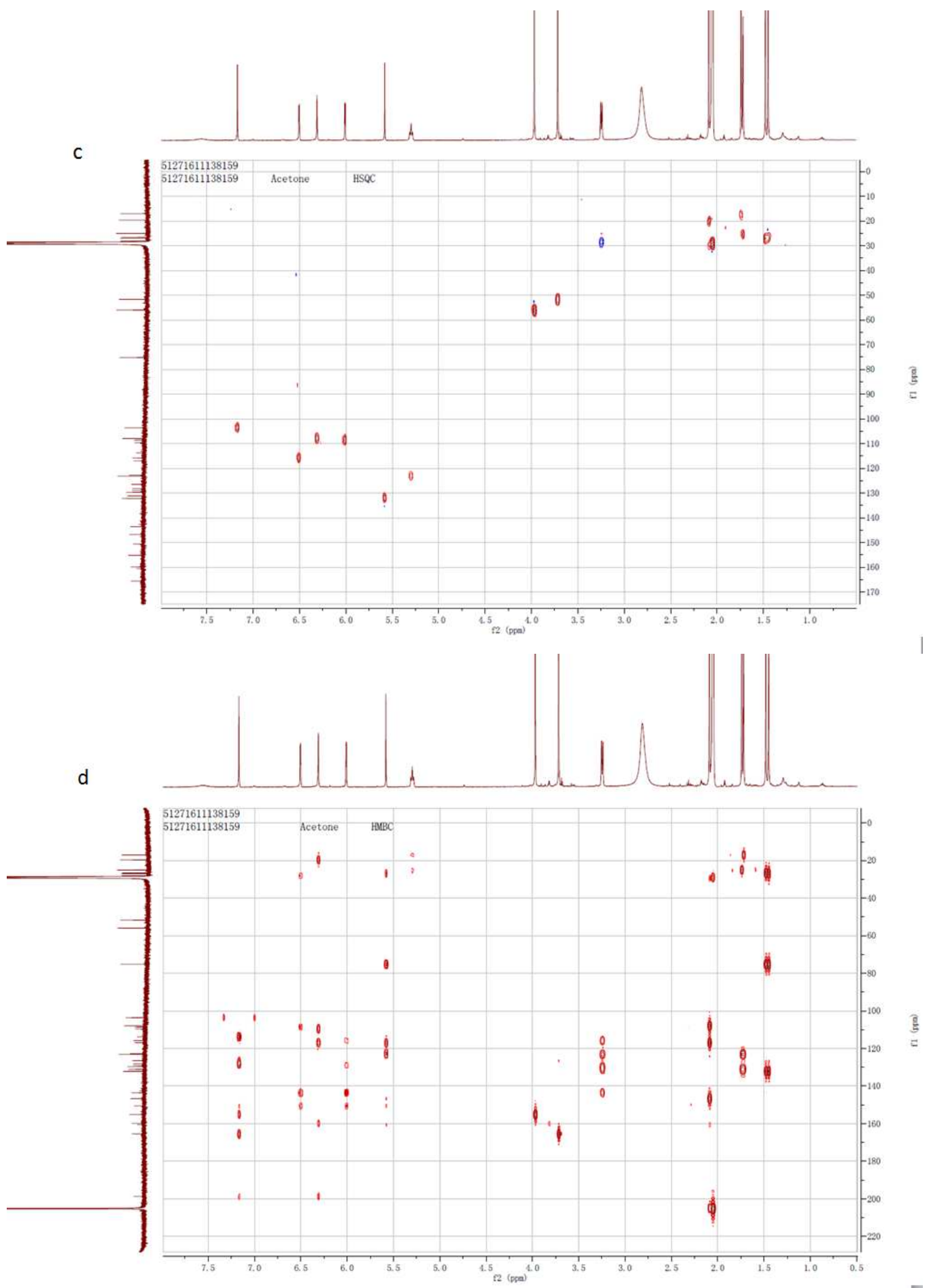

Figure S13. NMR analysis of 2 in acetone- $d_{6}$. (a) ${ }^{1} \mathrm{H}$ NMR spectrum. (b) ${ }^{13} \mathrm{C}$ NMR spectrum. (c) HSQC spectrum. (d) HMBC spectrum. 

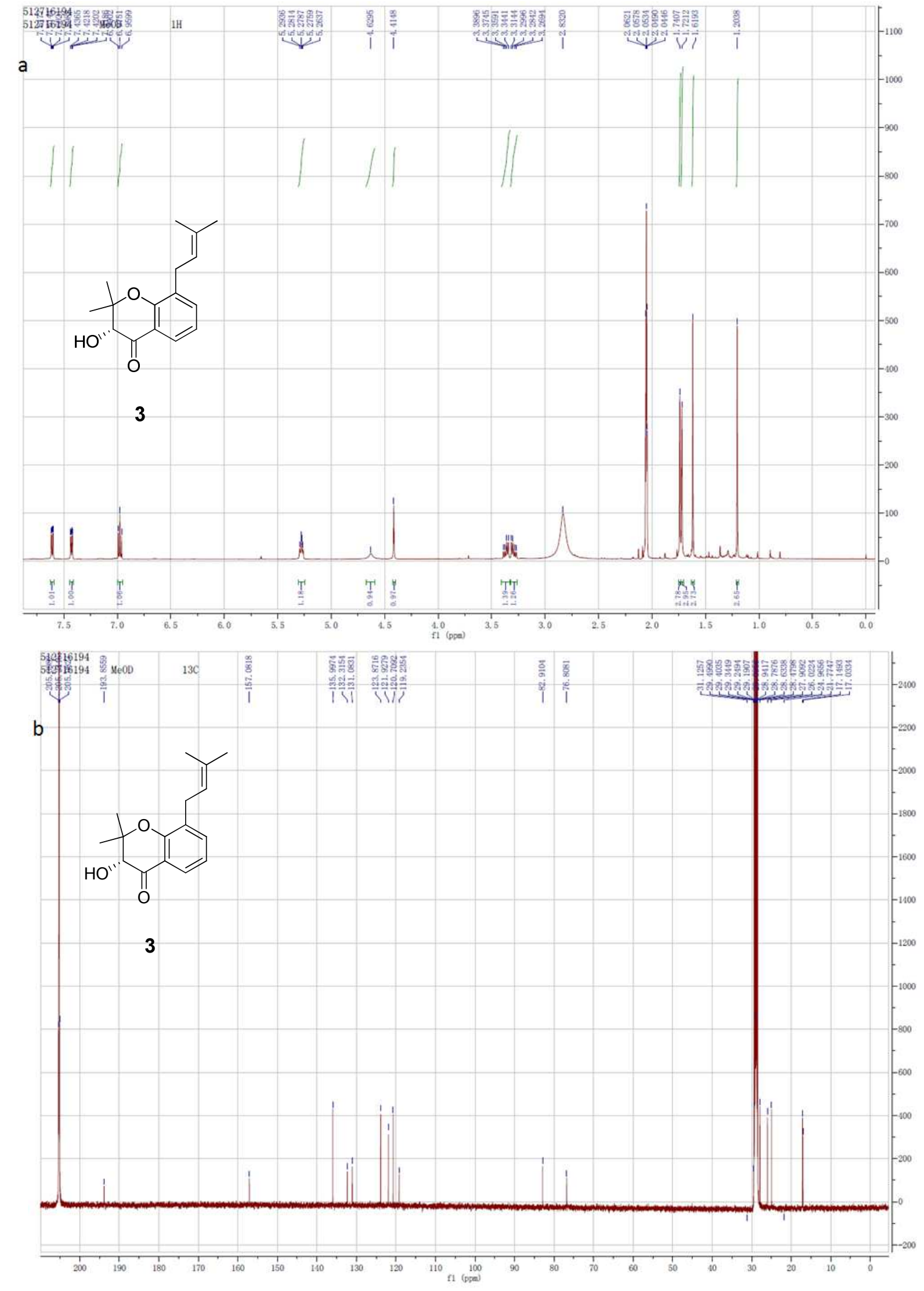

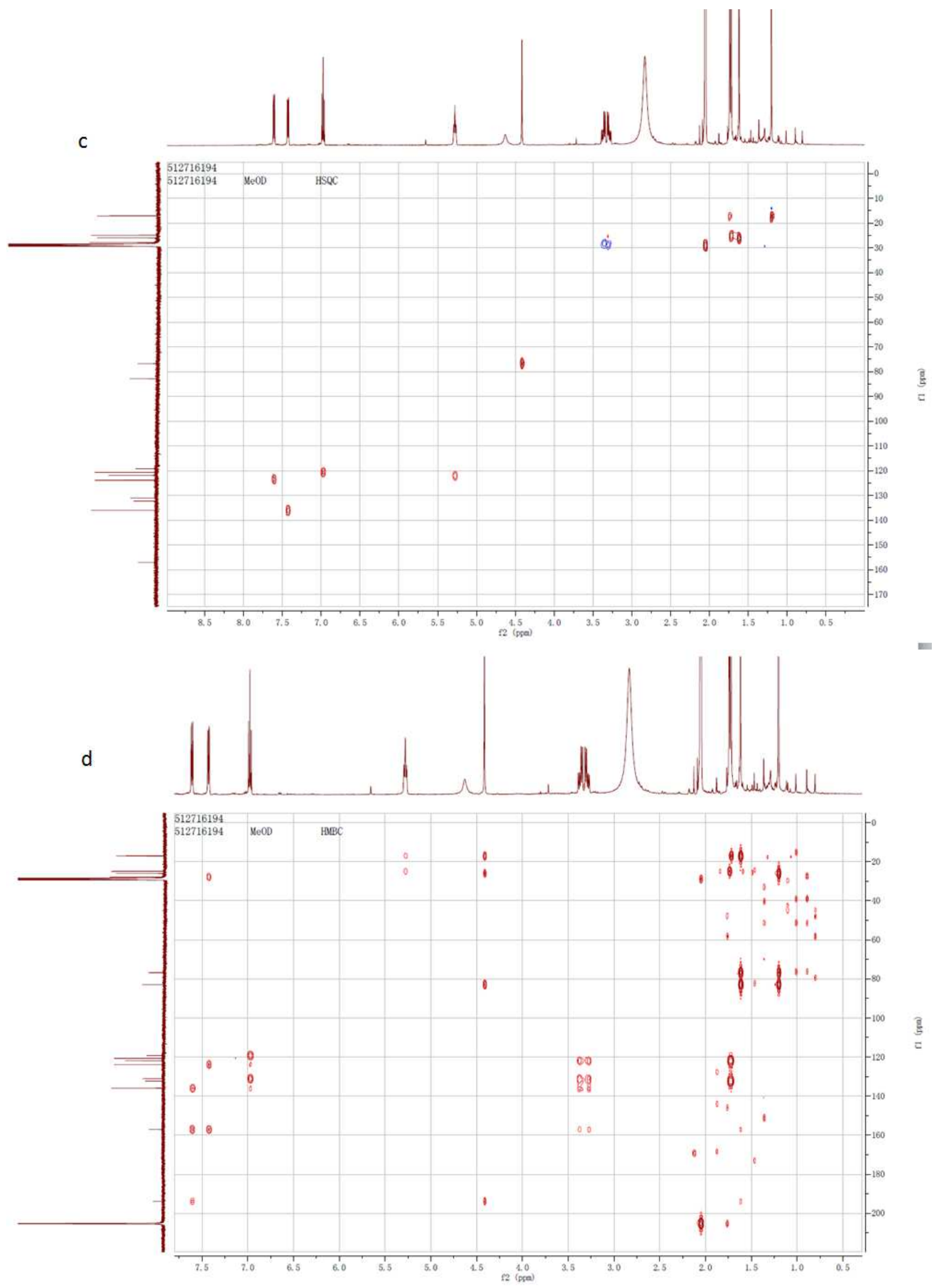


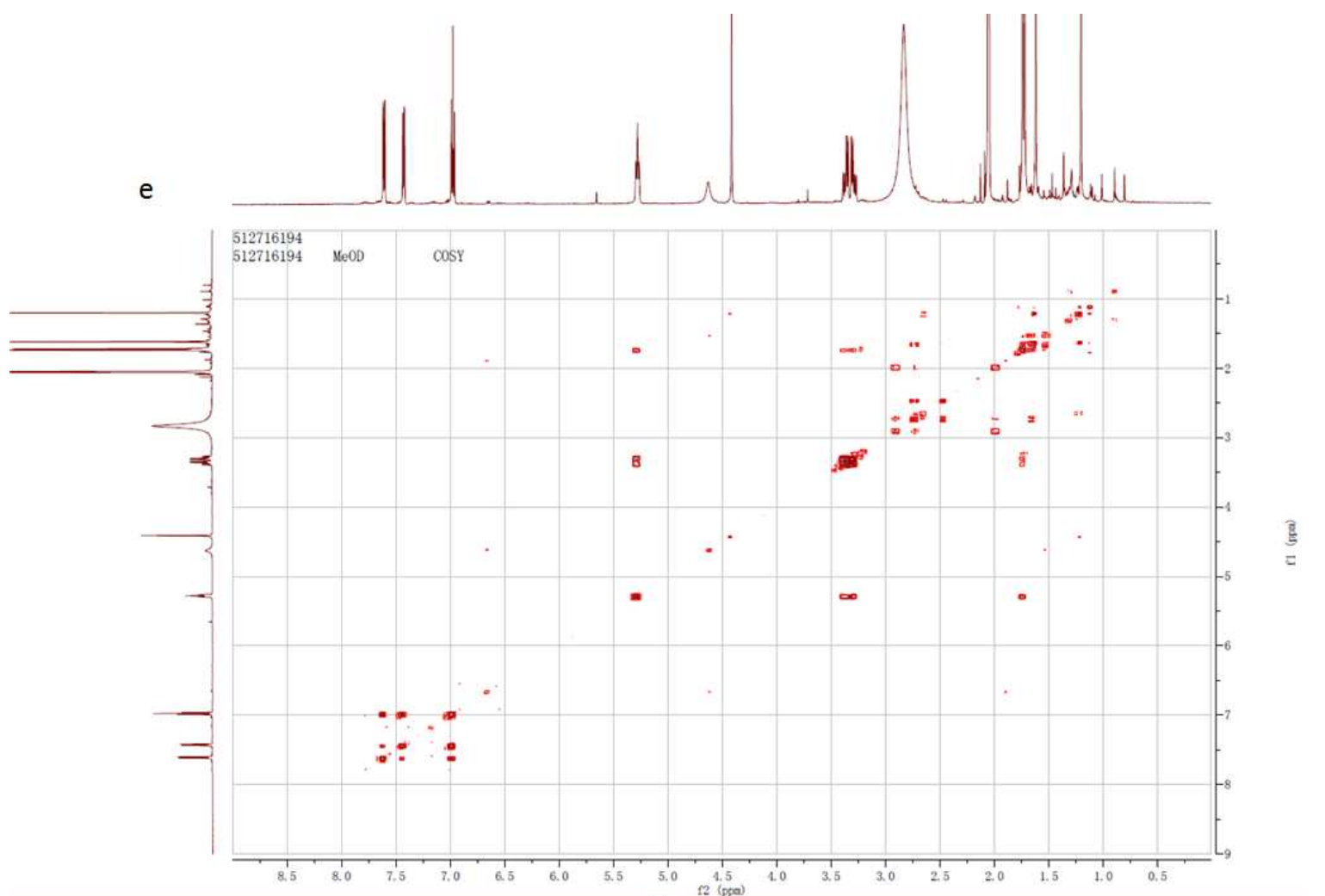

Figure S14. NMR analysis of 3 in acetone- $d_{6}$. (a) ${ }^{1} \mathrm{H}$ NMR spectrum. (b) ${ }^{13} \mathrm{C}$ NMR spectrum. (c) HSQC spectrum. (d) HMBC spectrum. (e) ${ }^{1} \mathrm{H}-{ }^{1} \mathrm{H}$ COSY spectrum. 

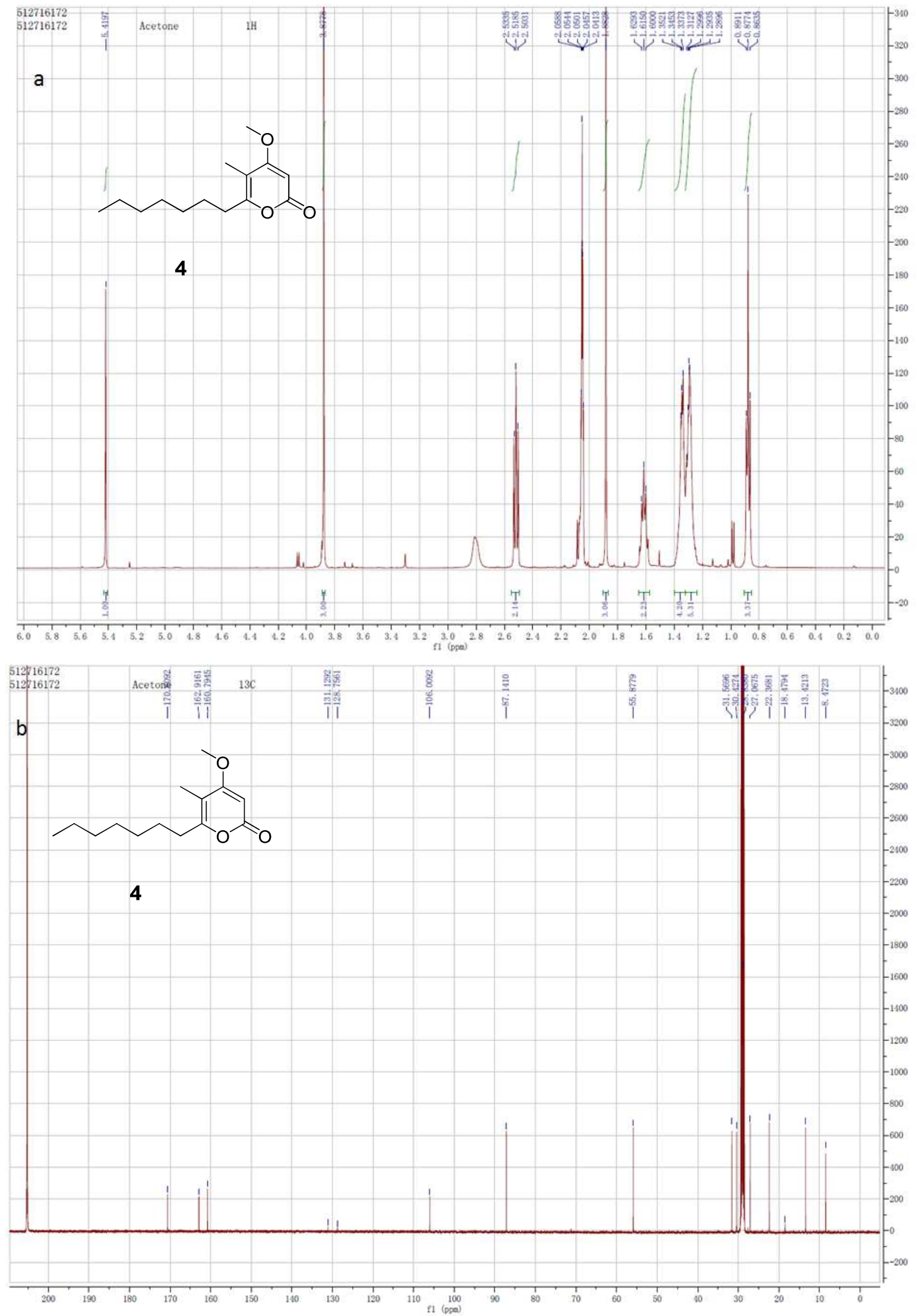

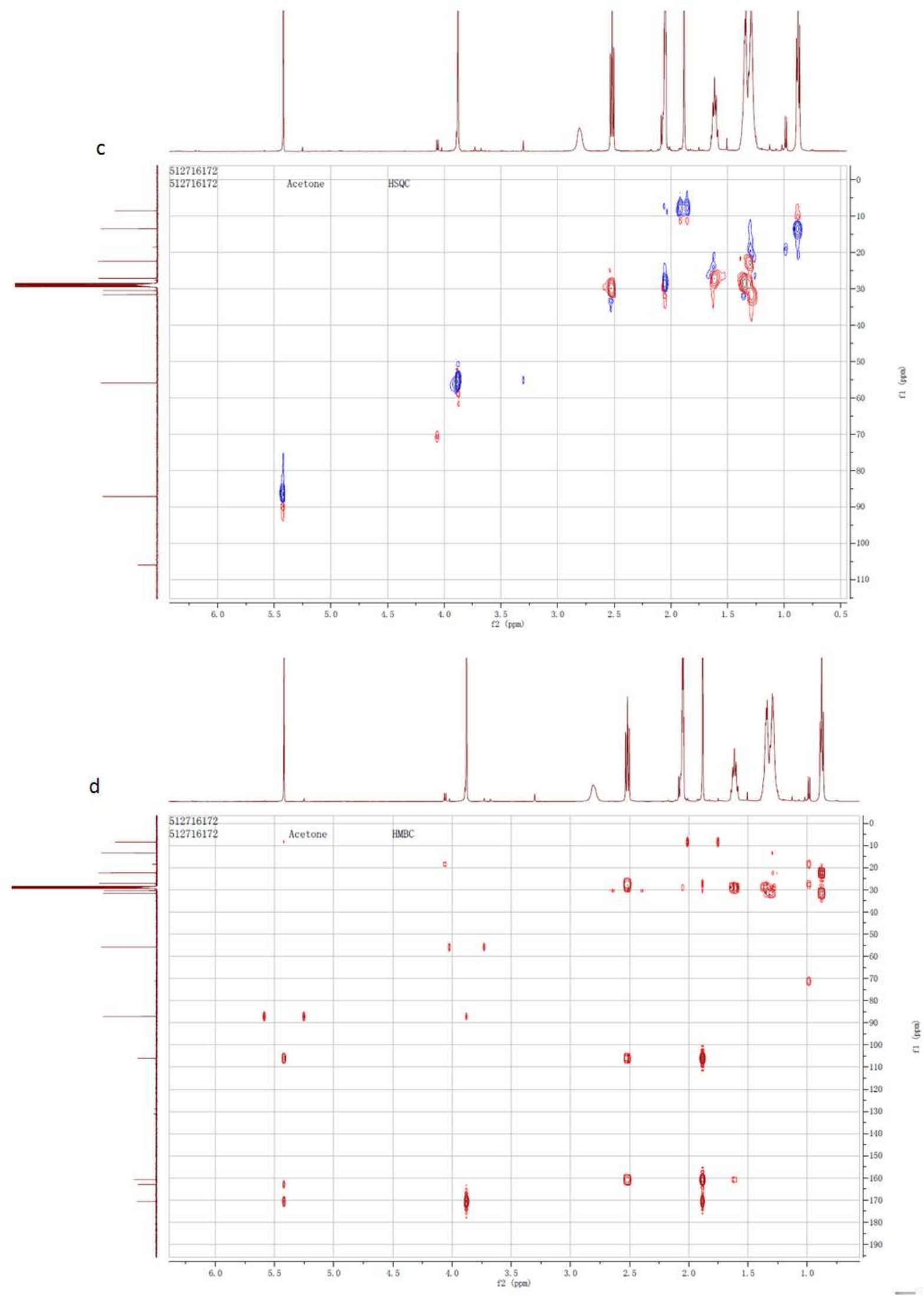


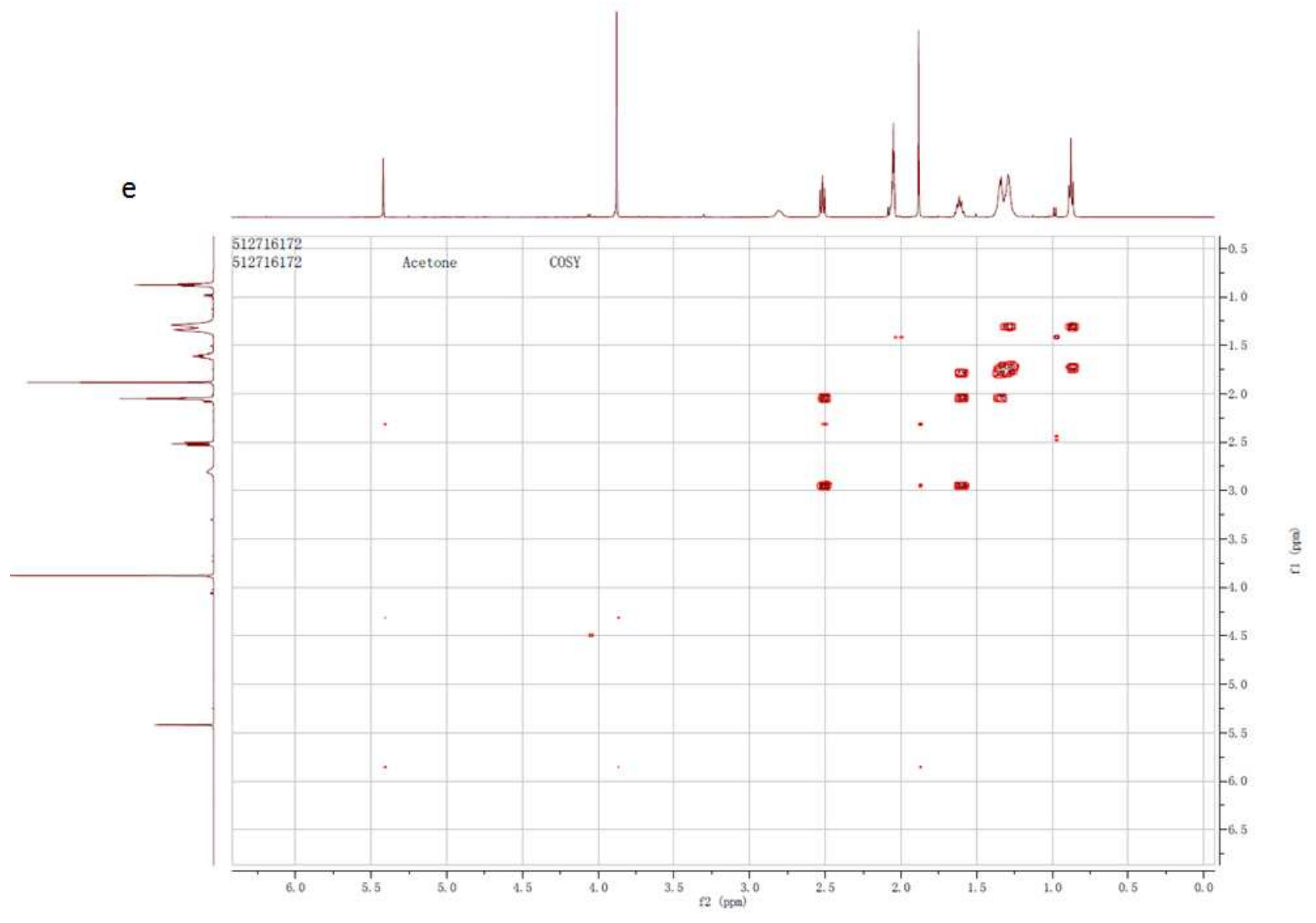

Figure S15. NMR analysis of 4 in acetone- $d_{6}$. (a) ${ }^{1} \mathrm{H}$ NMR spectrum. (b) ${ }^{13} \mathrm{C}$ NMR spectrum. (c) HSQC spectrum. (d) HMBC spectrum. (e) ${ }^{1} \mathrm{H}-{ }^{1} \mathrm{H}$ COSY spectrum. 

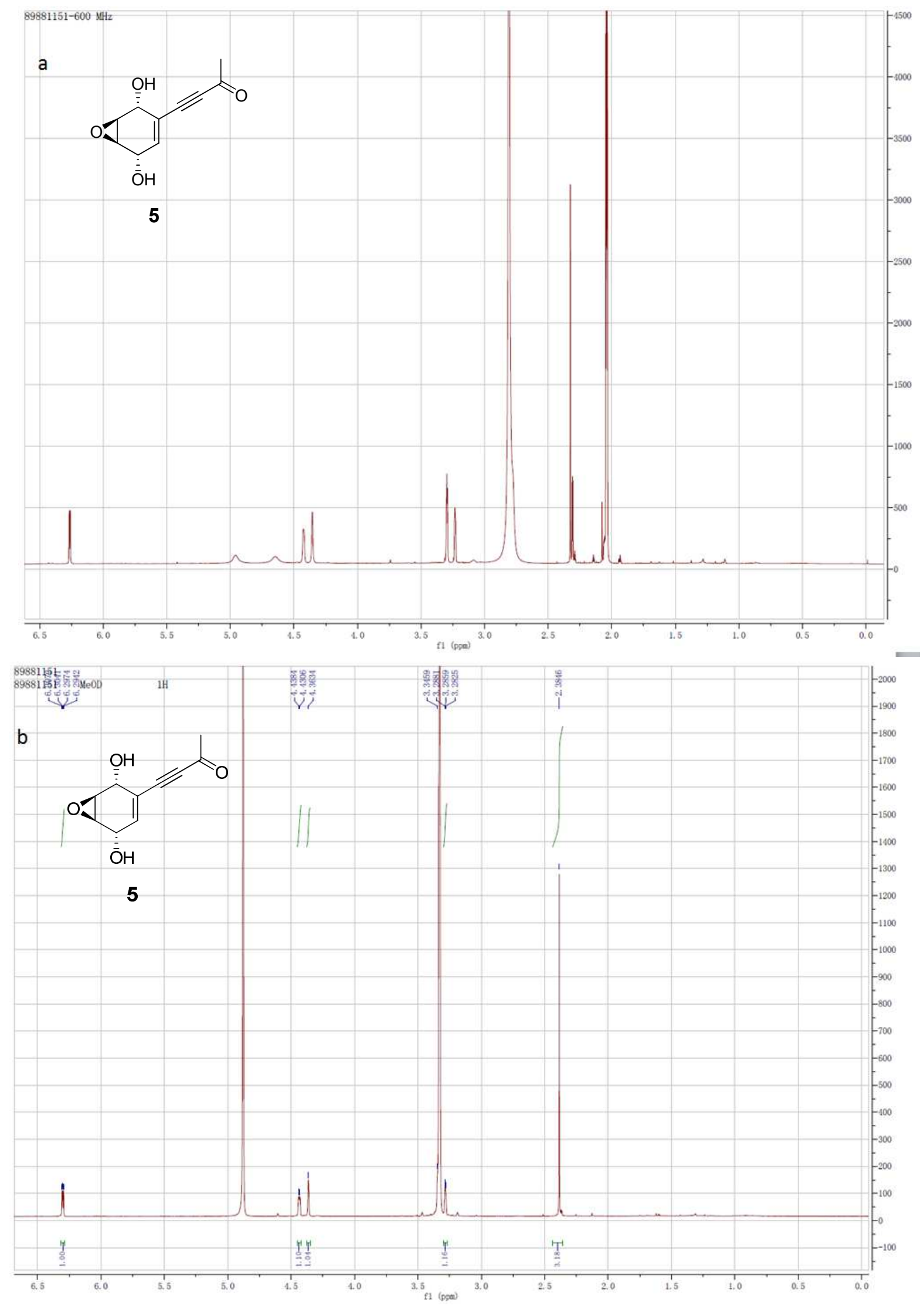

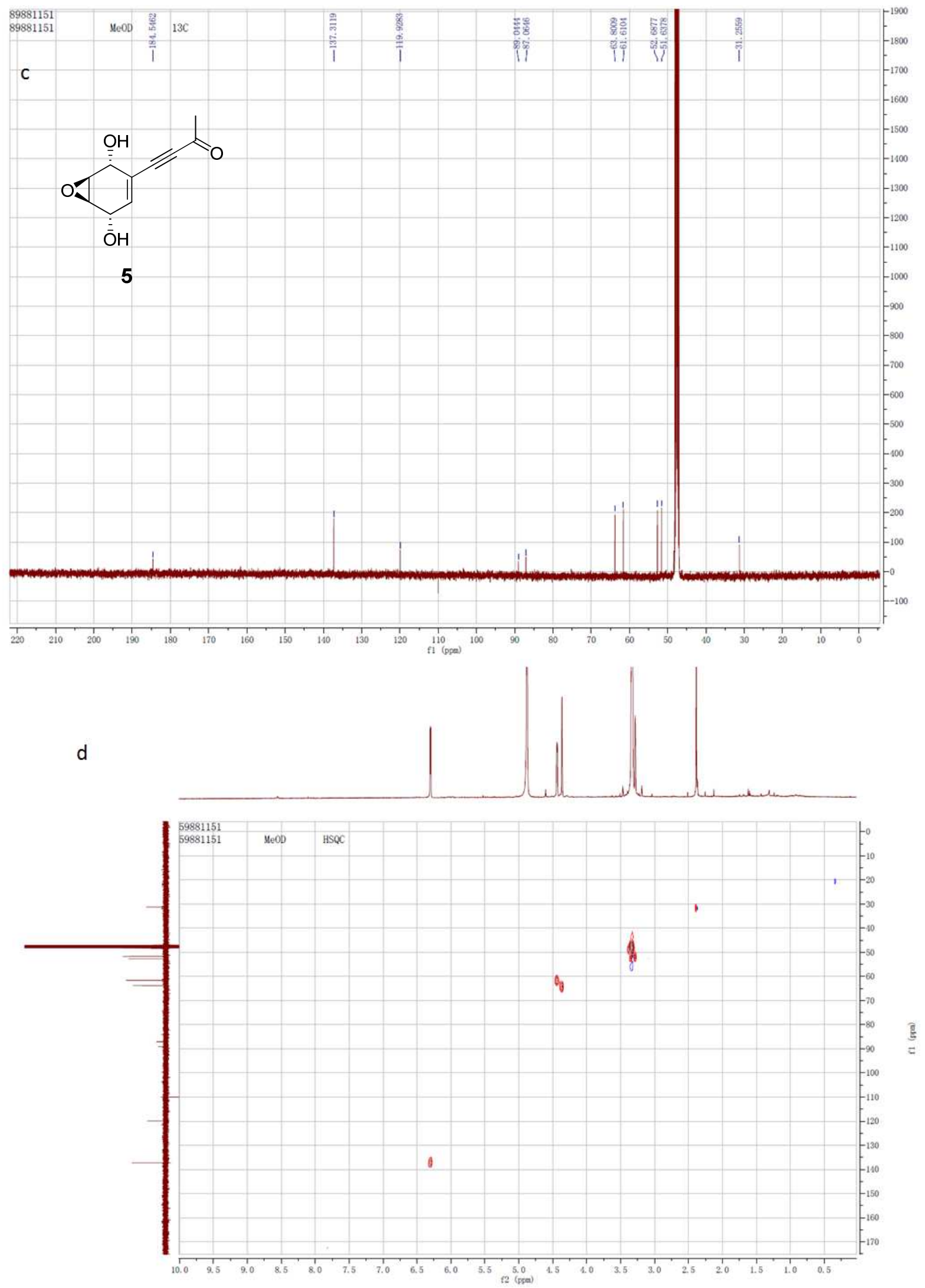


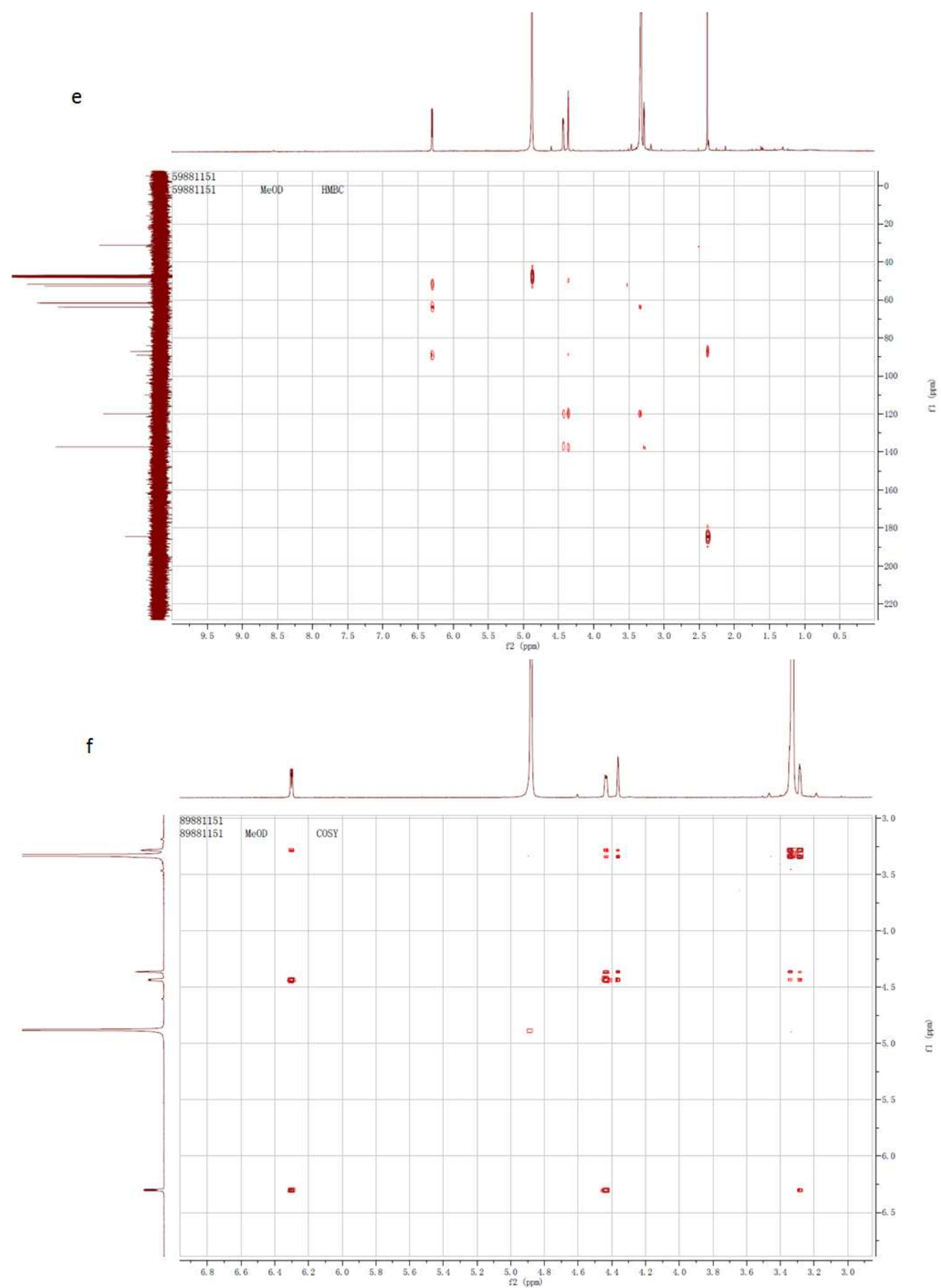

Figure S16. NMR analysis of 5 in methanol- $d_{4}$. (a) ${ }^{1} \mathrm{H}$ NMR spectrum in $600 \mathrm{MHz}$ (acetone- $d_{6}$ ). (b) ${ }^{1} \mathrm{H}$ NMR spectrum in $500 \mathrm{MHz}$. (c) ${ }^{13} \mathrm{C}$ NMR spectrum. (d) HSQC spectrum. (e) HMBC spectrum. (f) ${ }^{1} \mathrm{H}-{ }^{1} \mathrm{H}$ COSY spectrum. 

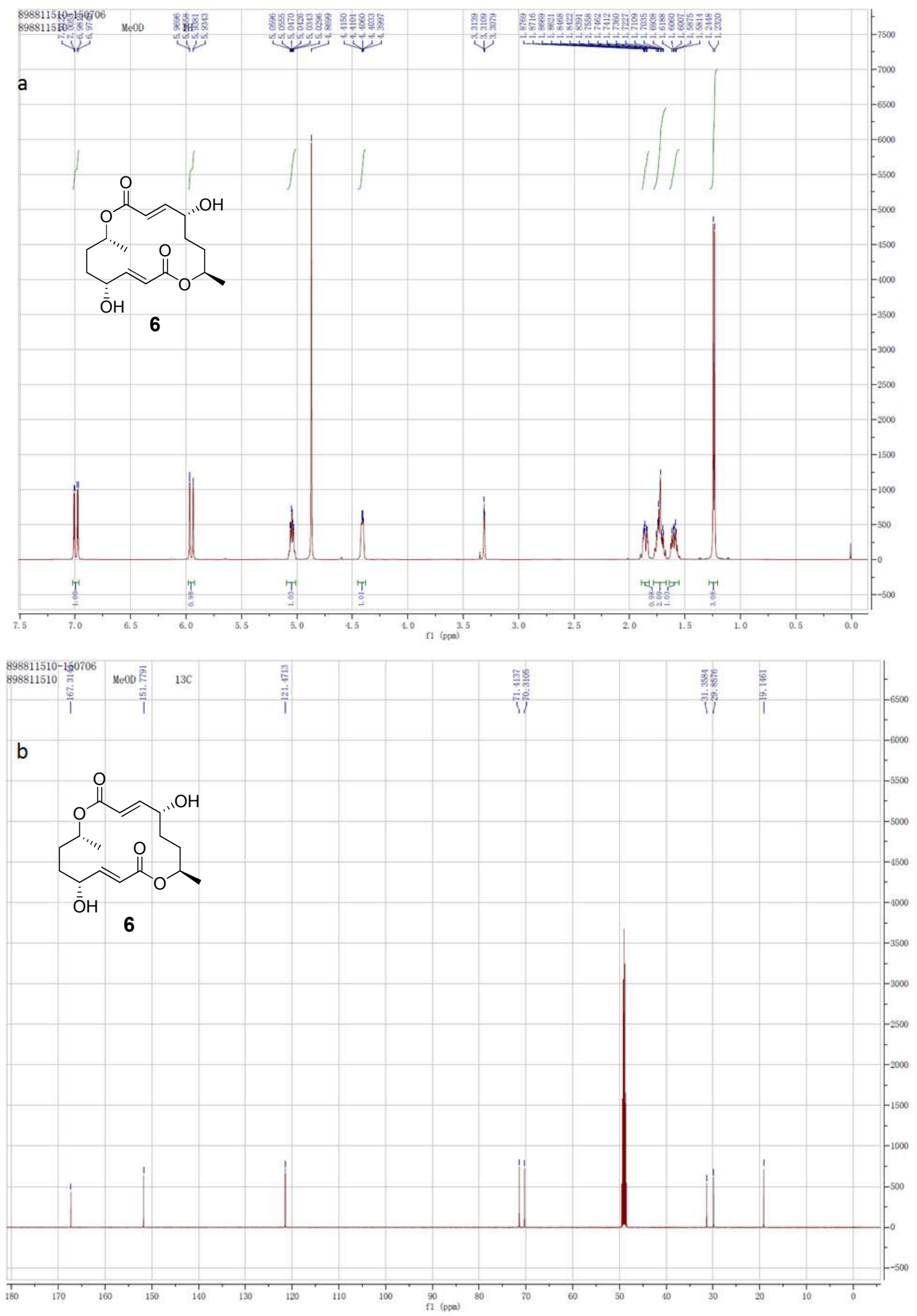

Figure S17. NMR analysis of 6 in methanol- $d_{4}$. (a) ${ }^{1} \mathrm{H}$ NMR spectrum. (b) ${ }^{13} \mathrm{C}$ NMR spectrum. 

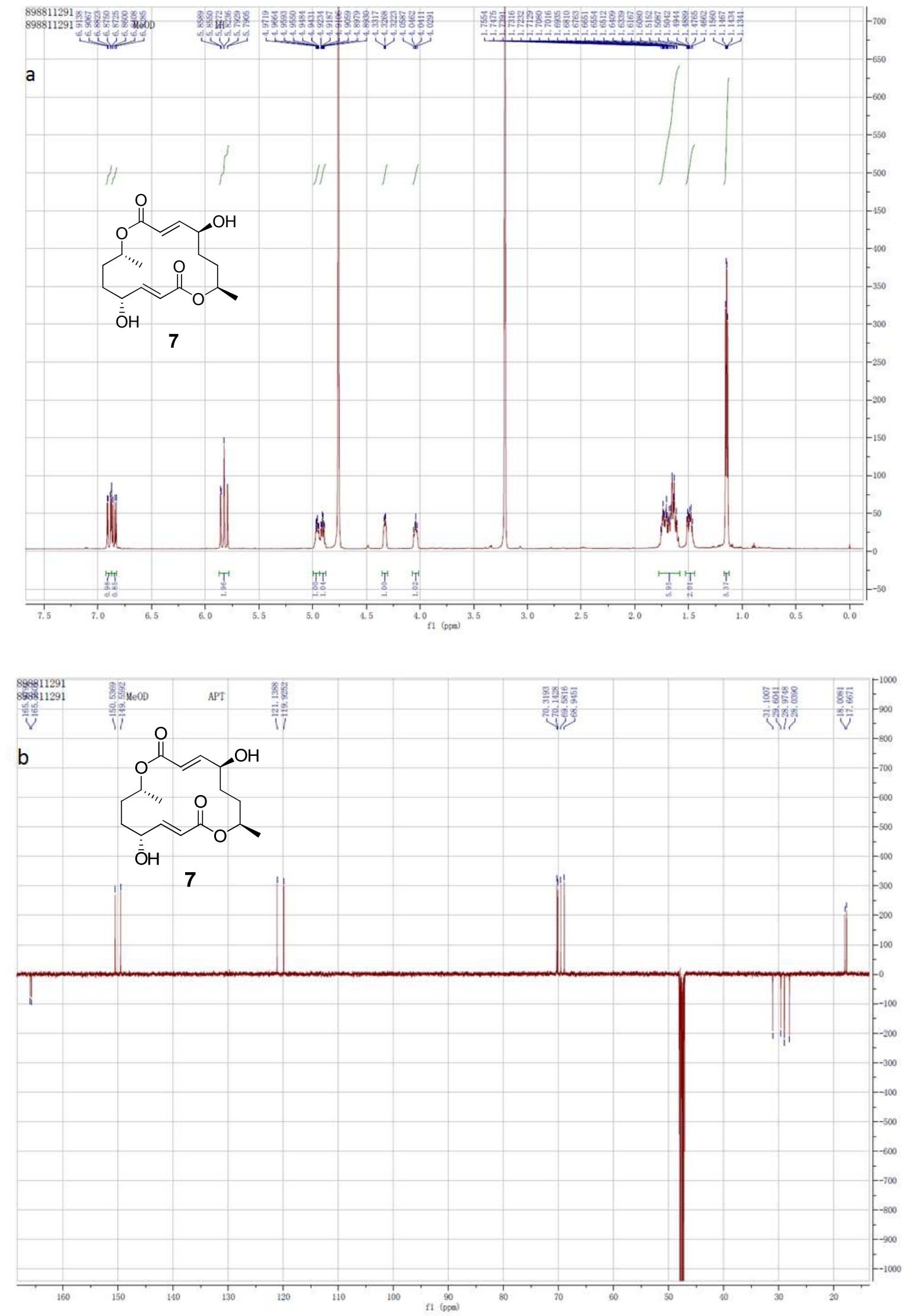


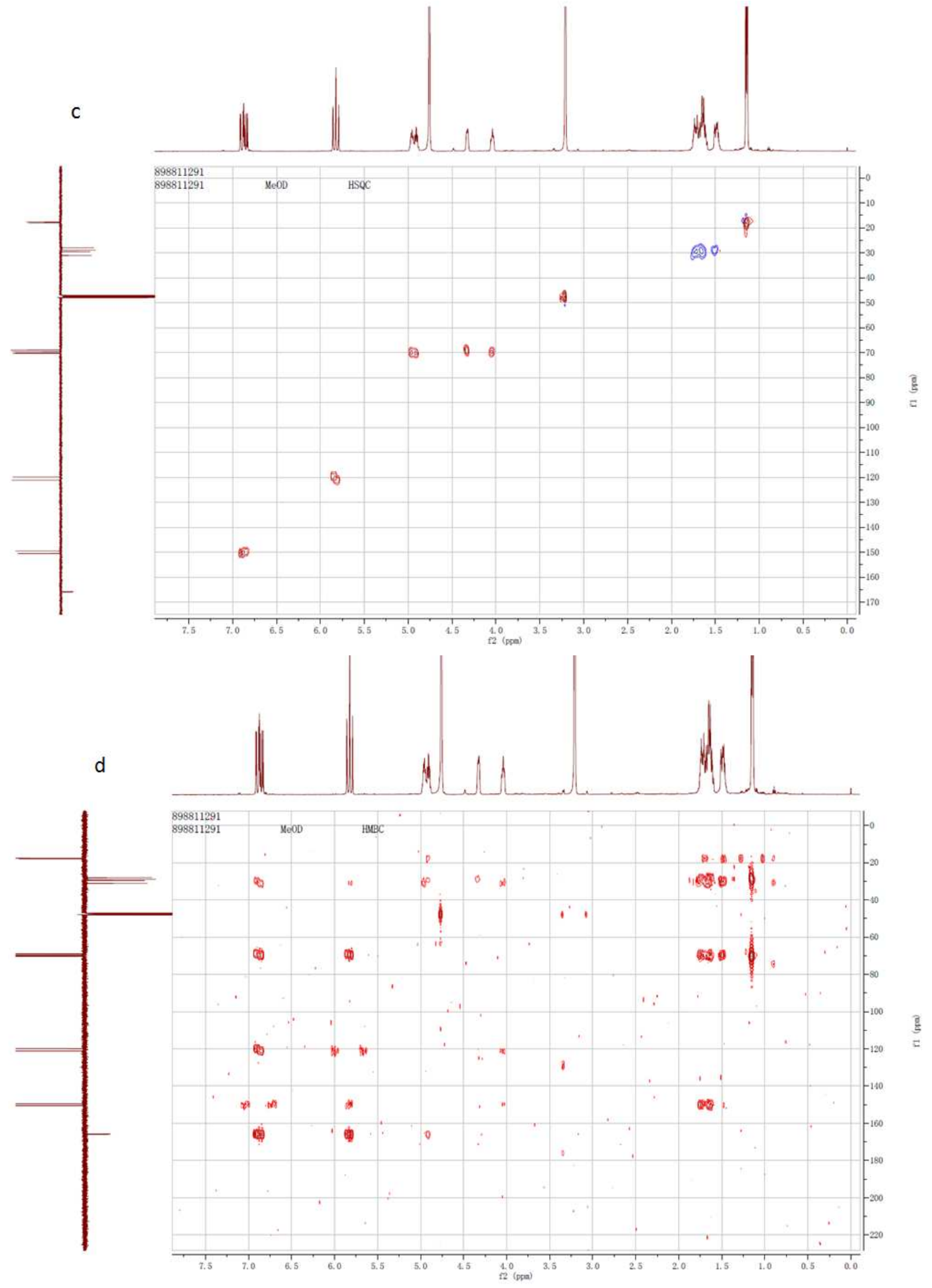




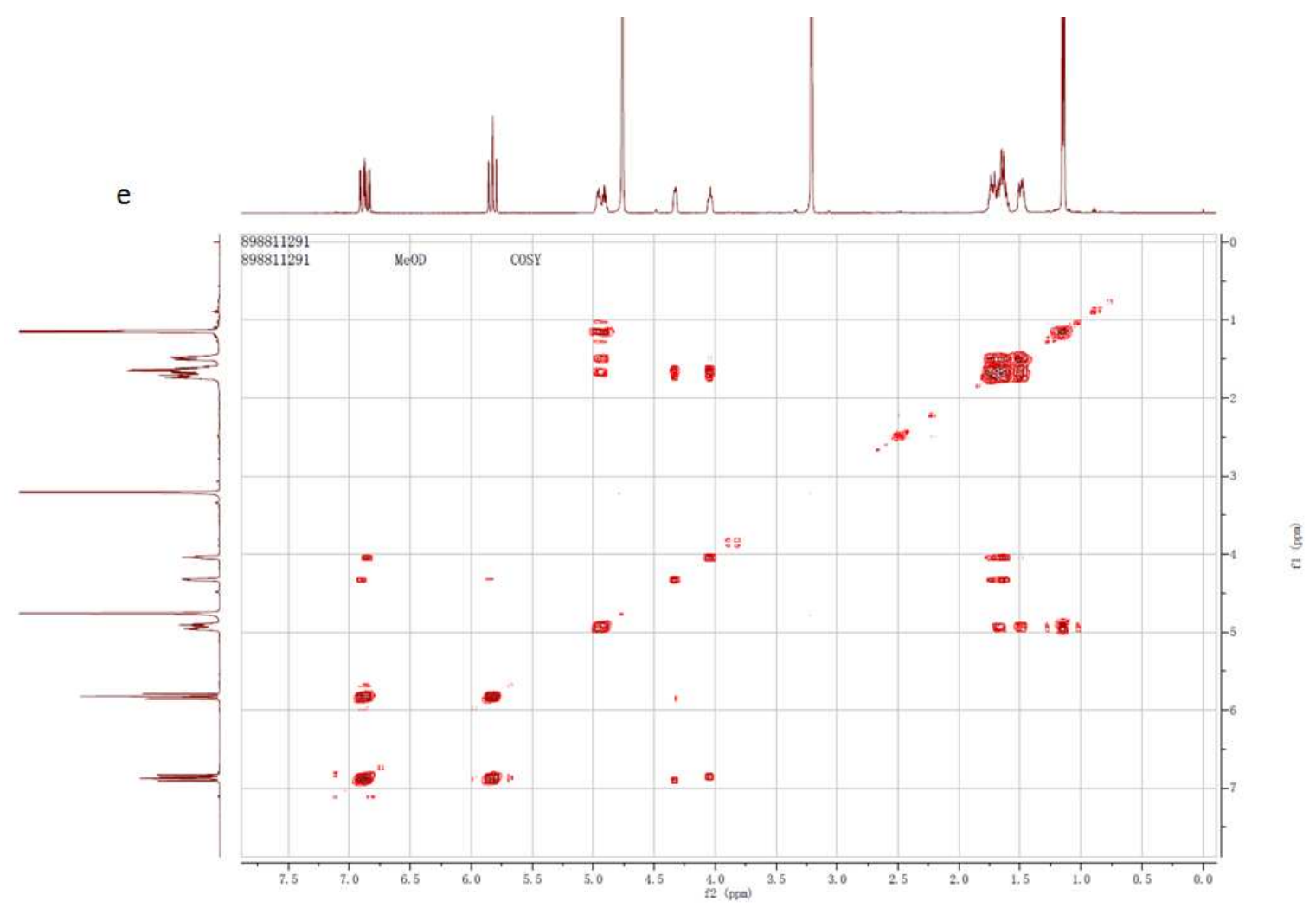

Figure S18. NMR analysis of 7 in methanol- $d_{4}$. (a) ${ }^{1} \mathrm{H}$ NMR spectrum. (b) ${ }^{13} \mathrm{C}$ NMR spectrum. (c) HSQC spectrum. (d) HMBC spectrum. (e) ${ }^{1} \mathrm{H}-{ }^{-1} \mathrm{H}$ COSY spectrum. 


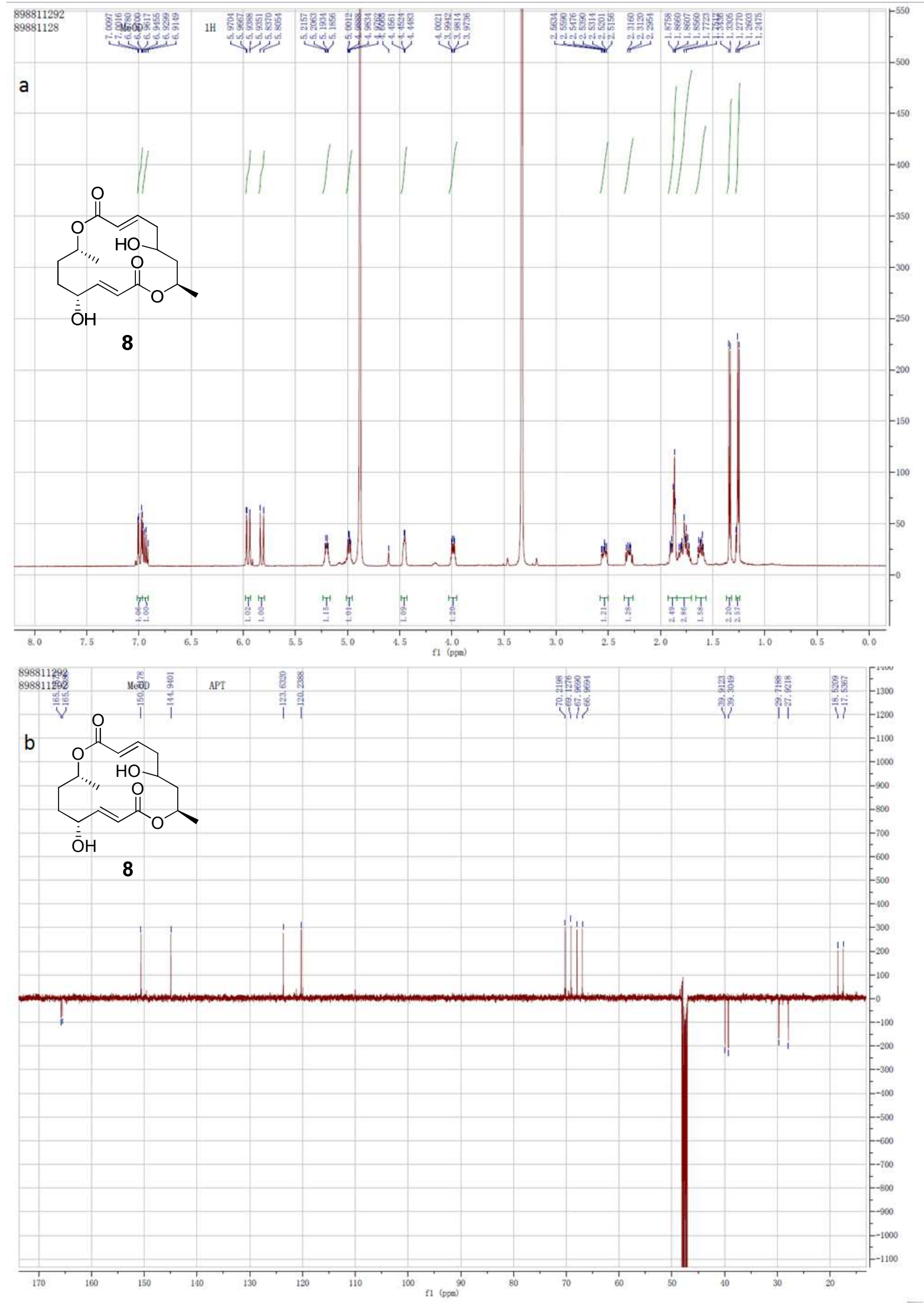



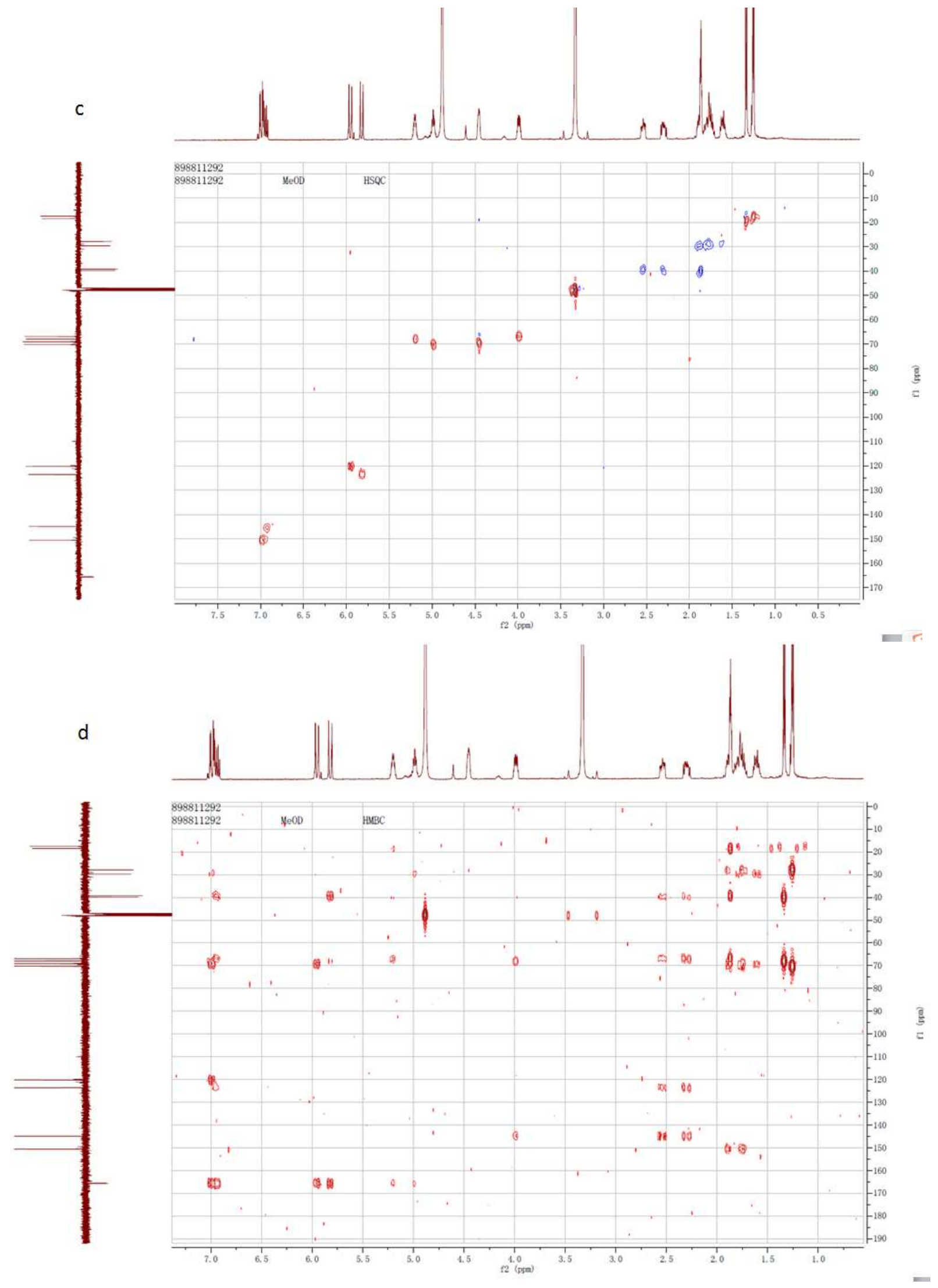


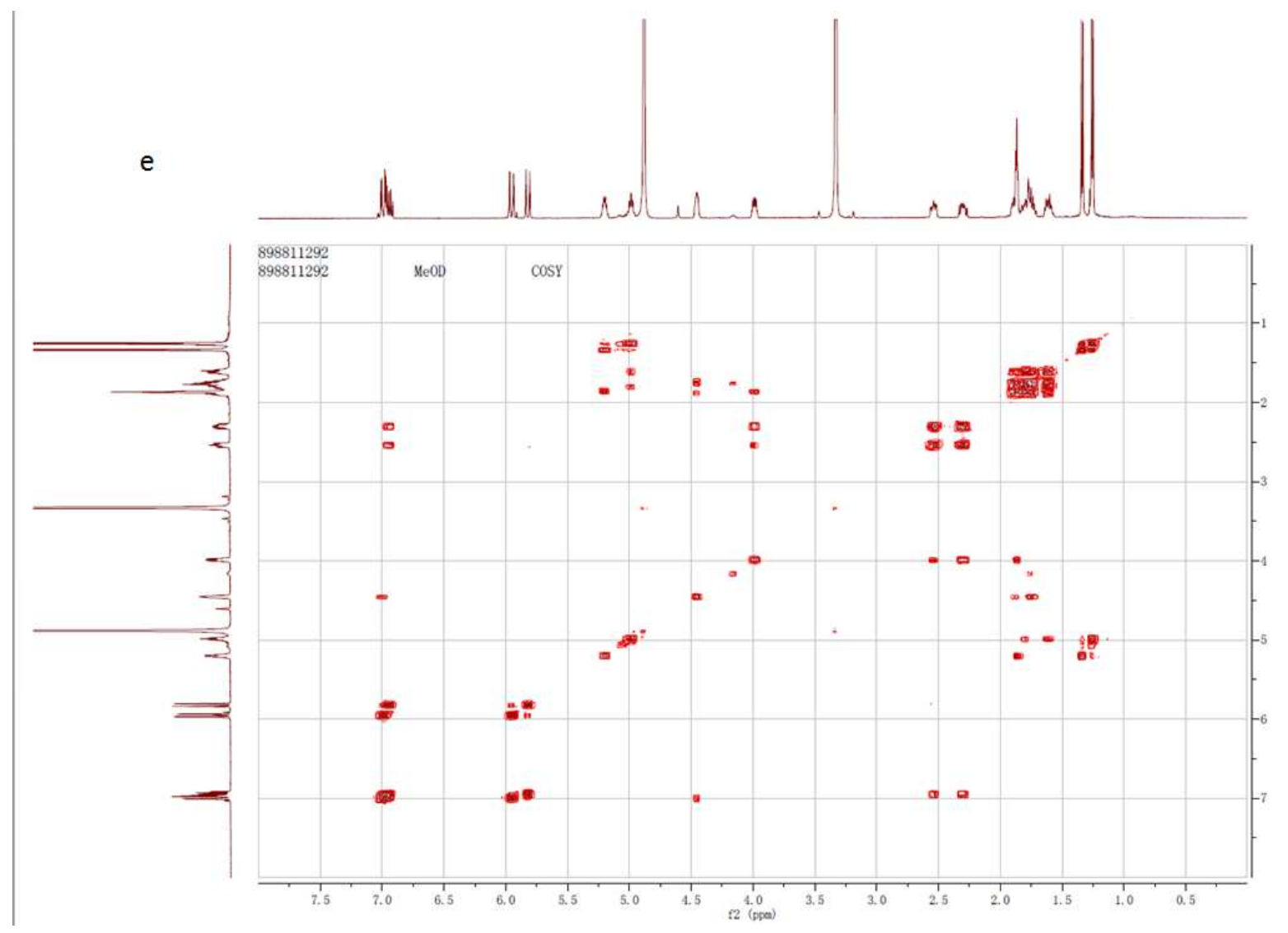

Figure S19. NMR analysis of 8 in methanol- $d_{4}$. (a) ${ }^{1} \mathrm{H}$ NMR spectrum. (b) ${ }^{13} \mathrm{C}$ NMR spectrum. (c) HSQC spectrum. (d) HMBC spectrum. (e) ${ }^{1} \mathrm{H}-{ }^{1} \mathrm{H}$ COSY spectrum. 

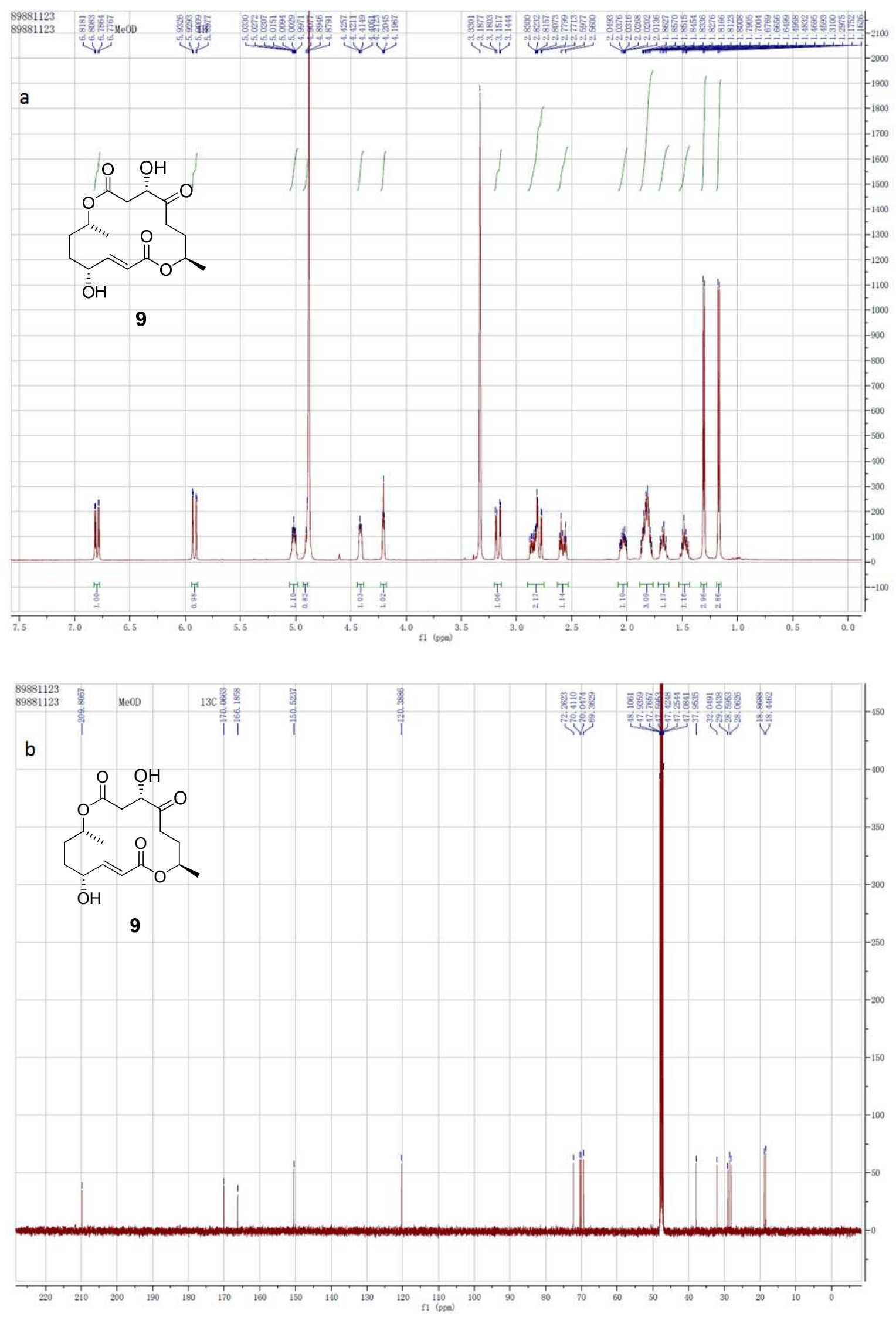


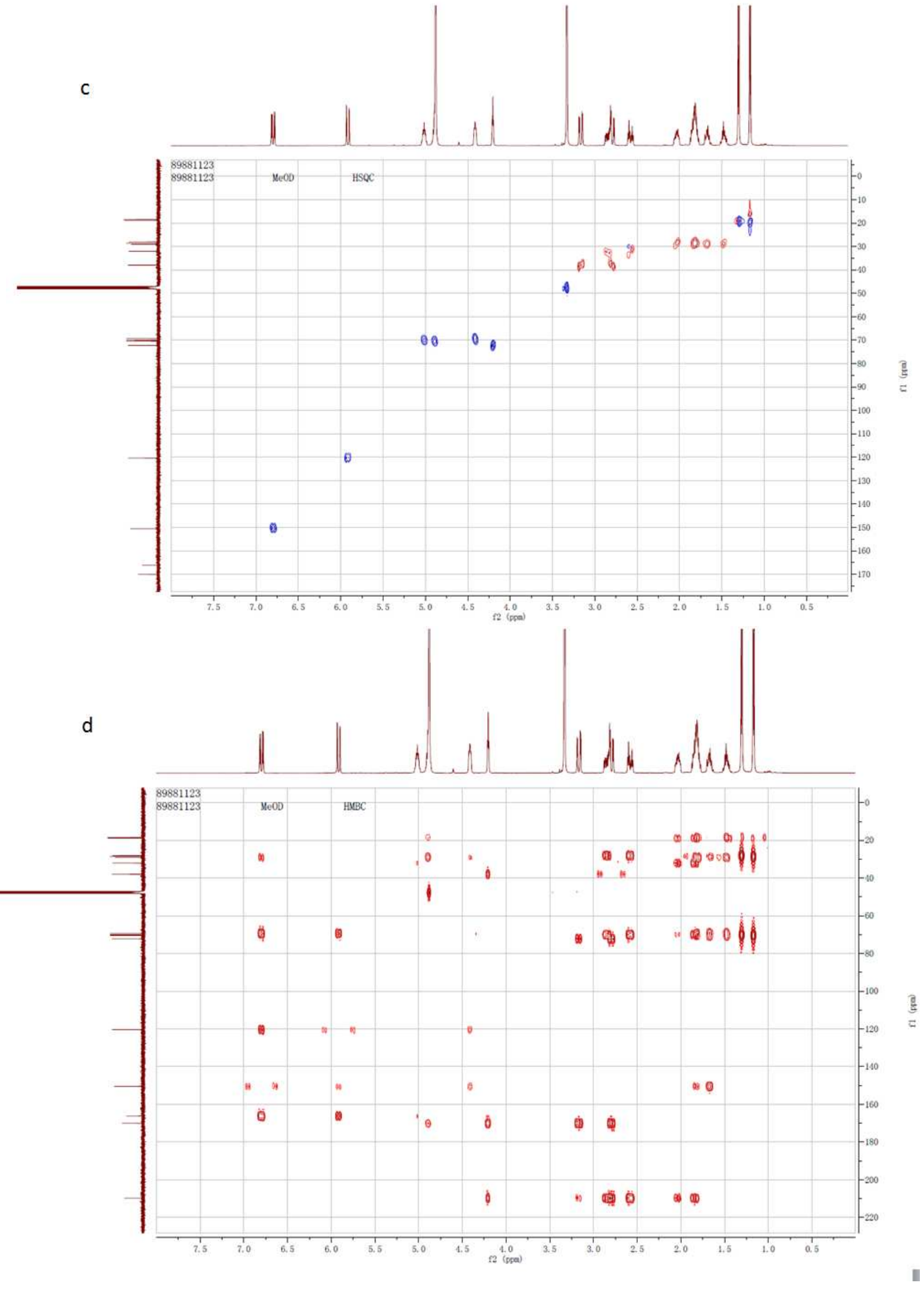




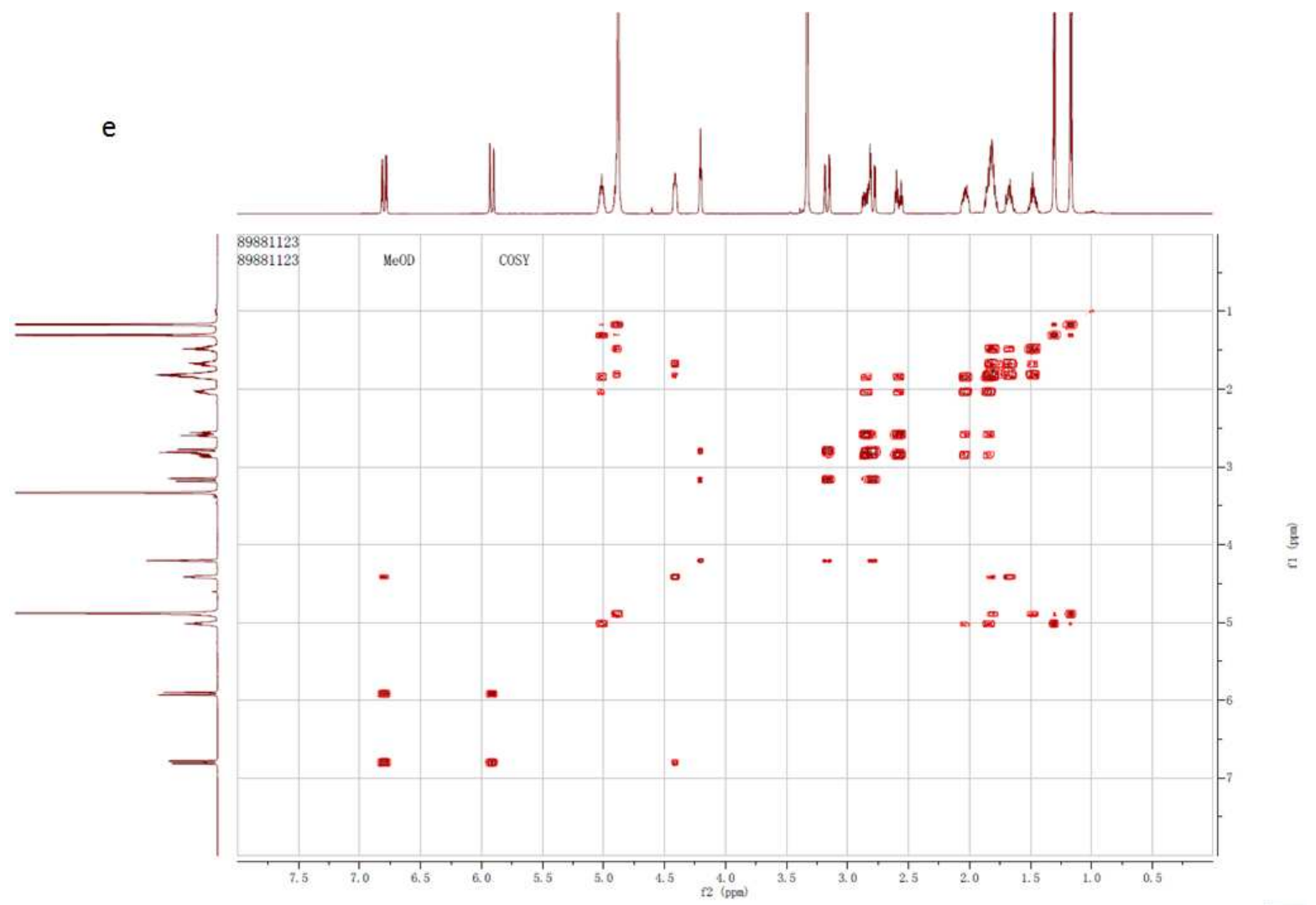

Figure S20. NMR analysis of 9 in methanol- $d_{4}$. (a) ${ }^{1} \mathrm{H}$ NMR spectrum. (b) ${ }^{13} \mathrm{C}$ NMR spectrum. (c) HSQC spectrum. (d) HMBC spectrum. (e) ${ }^{1} \mathrm{H}-{ }^{-1} \mathrm{H}$ COSY spectrum. 


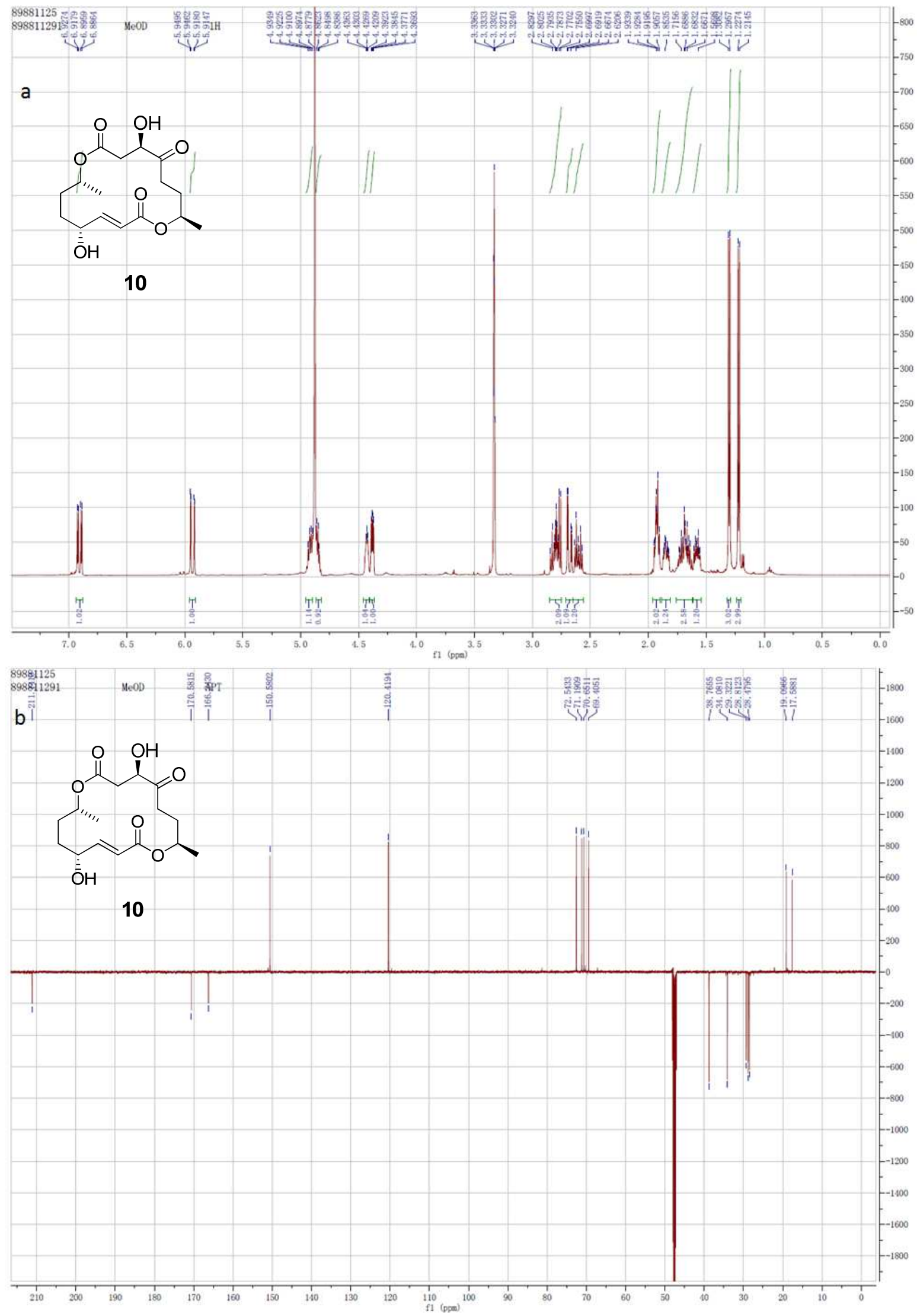




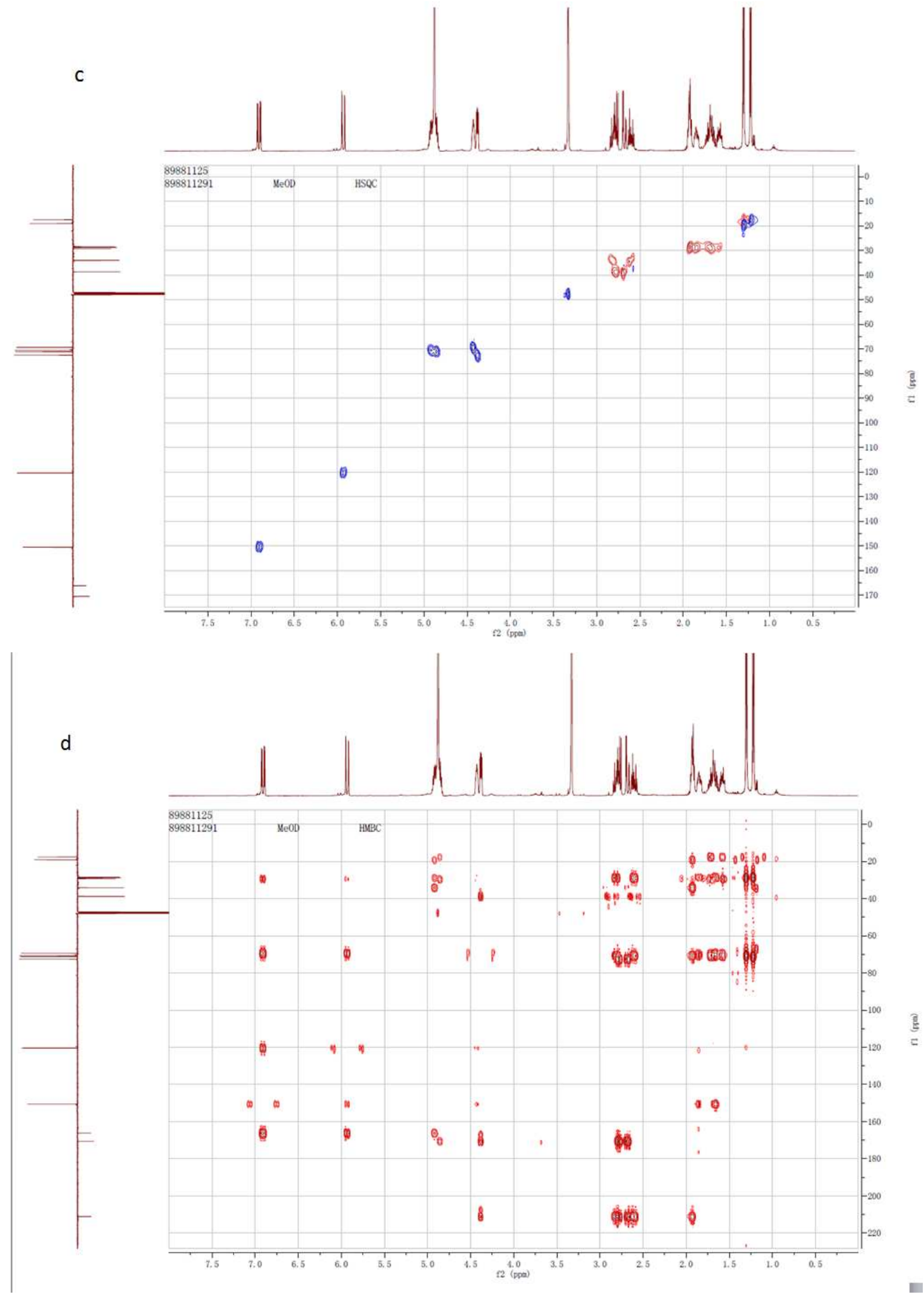




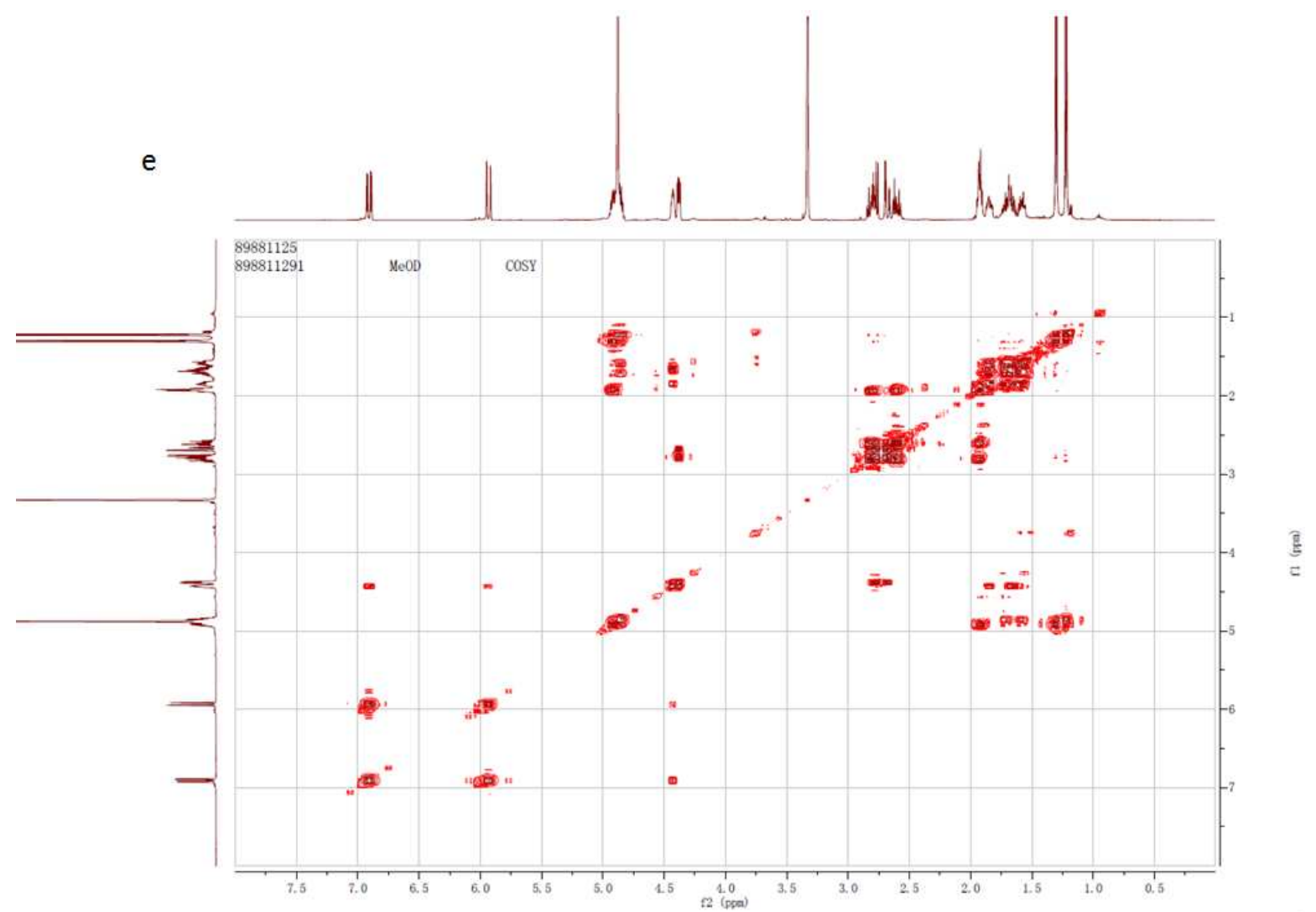

Figure S21. NMR analysis of $\mathbf{1 0}$ in methanol- $d_{4}$. (a) ${ }^{1} \mathrm{H}$ NMR spectrum. (b) ${ }^{13} \mathrm{C}$ NMR spectrum. (c) HSQC spectrum. (d) $\mathrm{HMBC}$ spectrum. (e) ${ }^{1} \mathrm{H}-{ }^{-} \mathrm{H}$ COSY spectrum. 

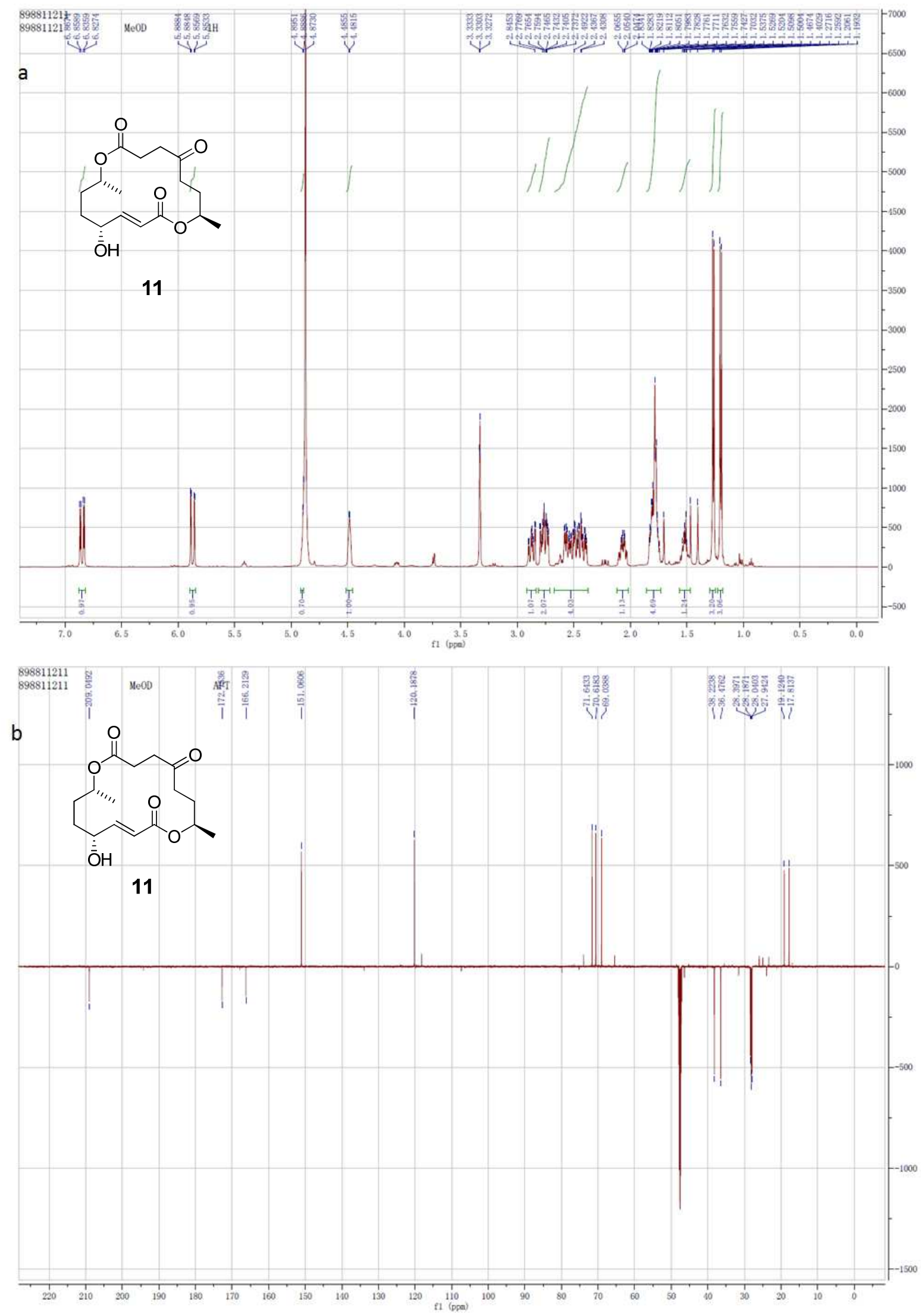

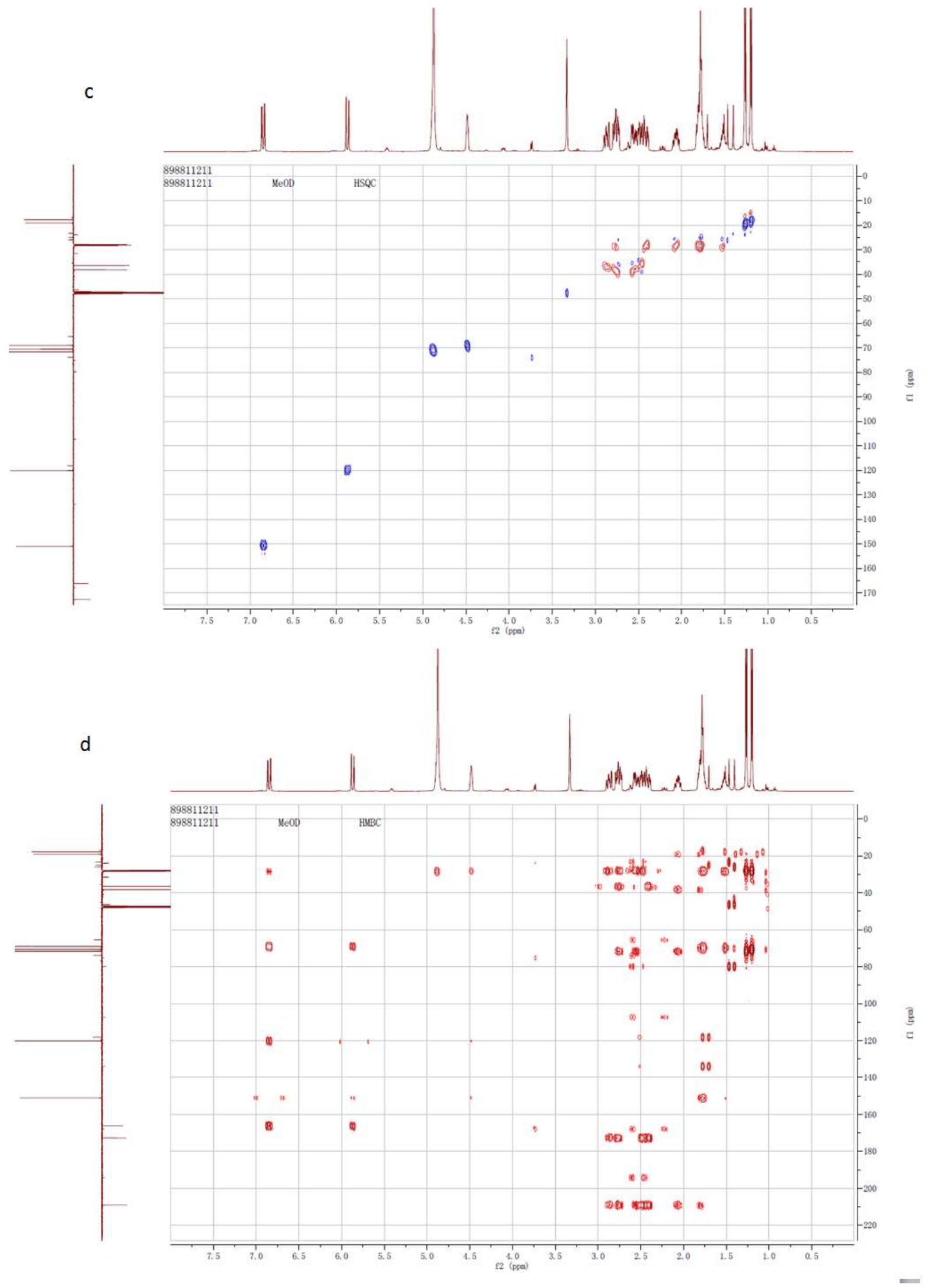


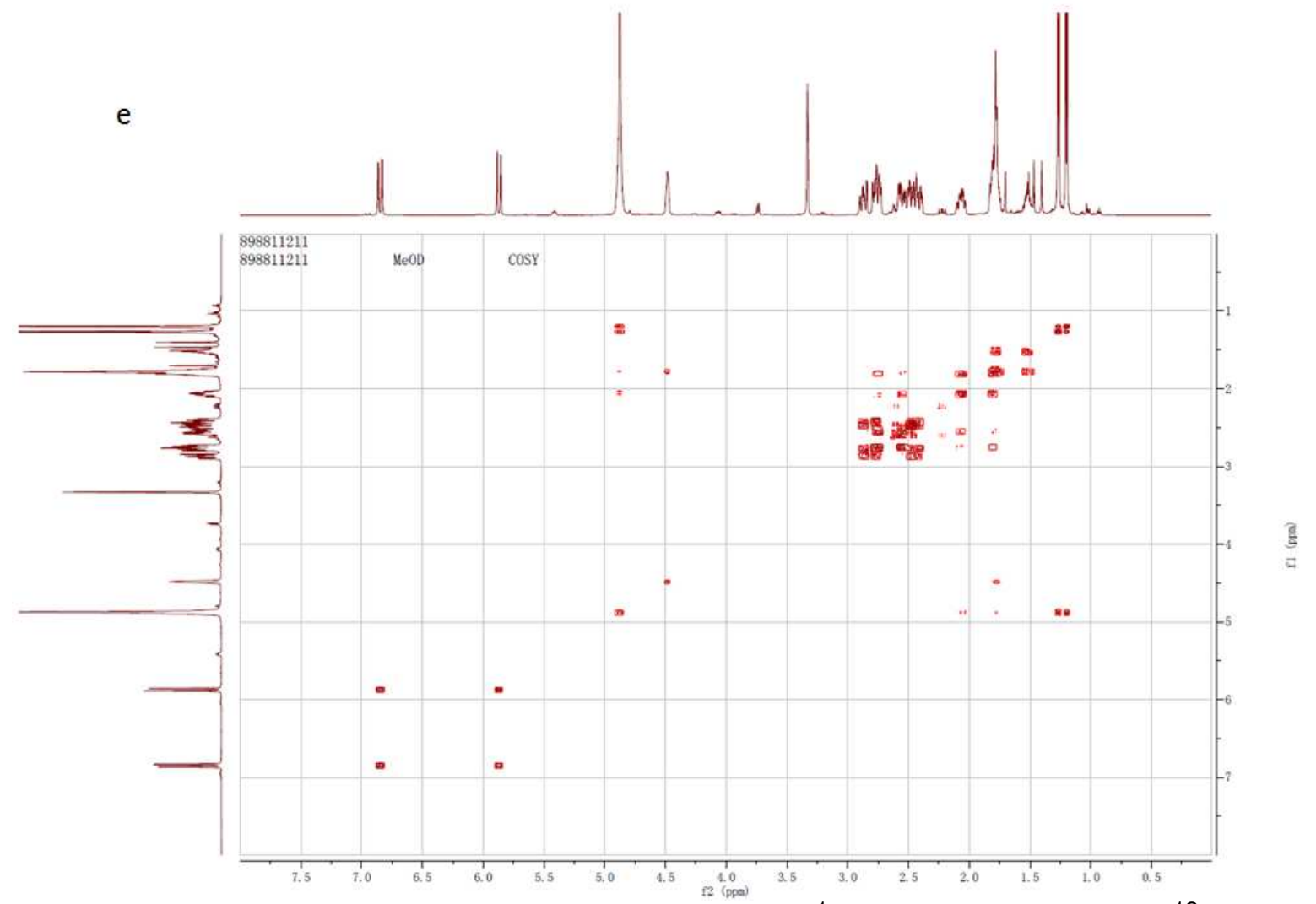

Figure S22. NMR analysis of 11 in methanol- $d_{4}$. (a) ${ }^{1} \mathrm{H}$ NMR spectrum. (b) ${ }^{13} \mathrm{C}$ NMR spectrum. (c) HSQC spectrum. (d) HMBC spectrum. (e) ${ }^{1} \mathrm{H}-{ }^{-1} \mathrm{H}$ COSY spectrum. 


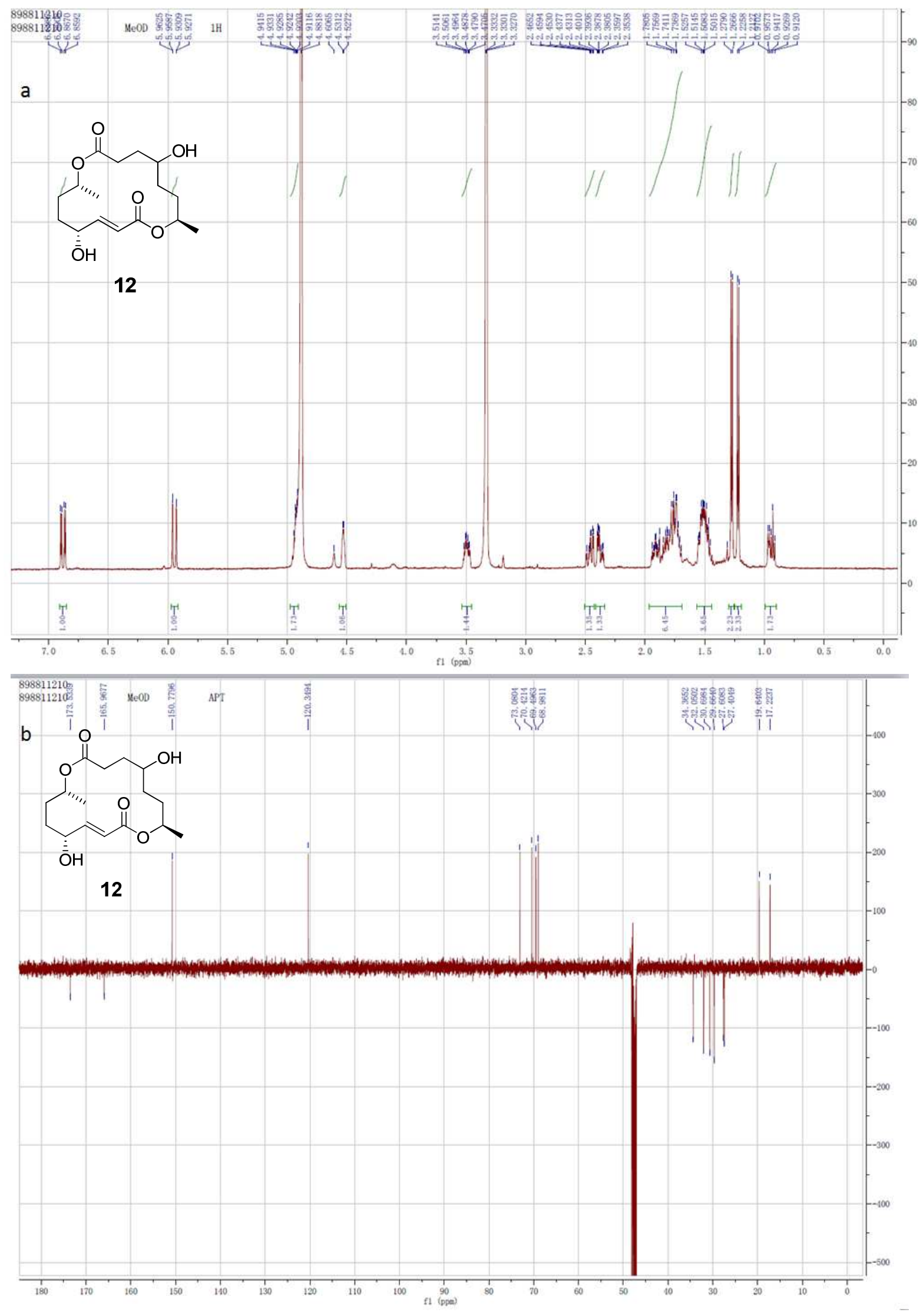




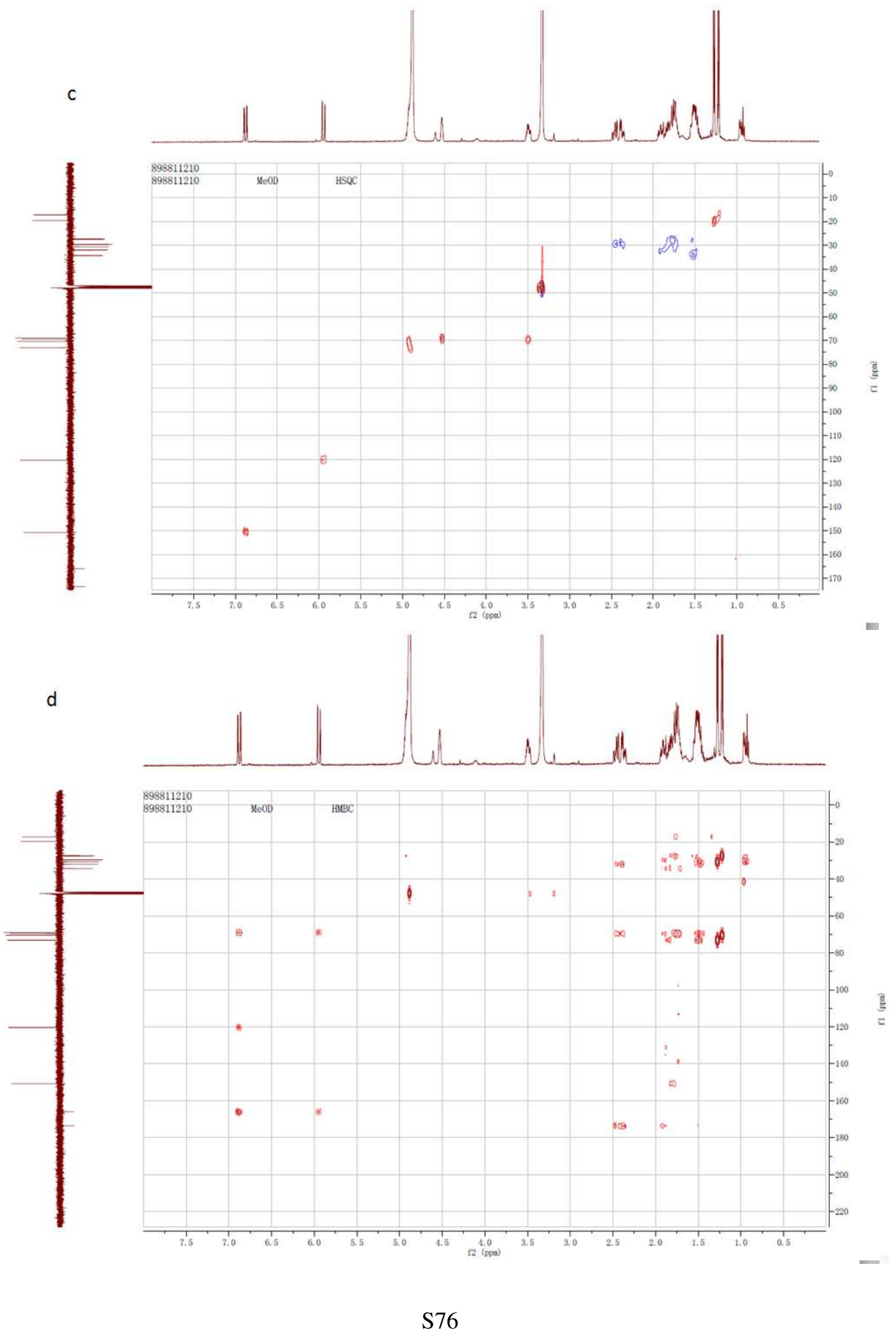




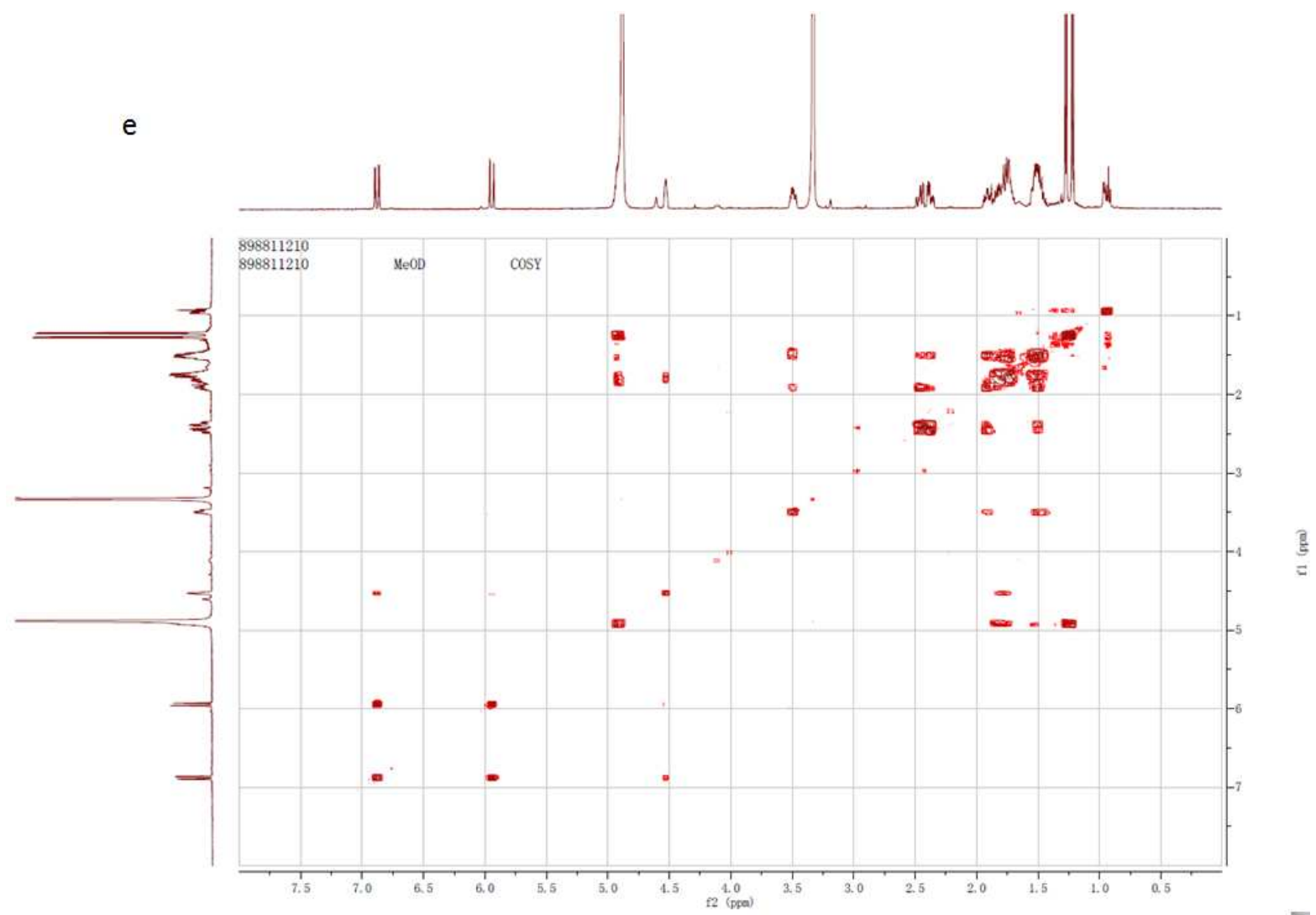

Figure S23. NMR analysis of 12 in methanol- $d_{4 .}$ (a) ${ }^{1} \mathrm{H}$ NMR spectrum. (b) ${ }^{13} \mathrm{C}$ NMR spectrum. (c) HSQC spectrum. (d) HMBC spectrum. (e) ${ }^{1} \mathrm{H}-{ }^{-1} \mathrm{H}$ COSY spectrum. 

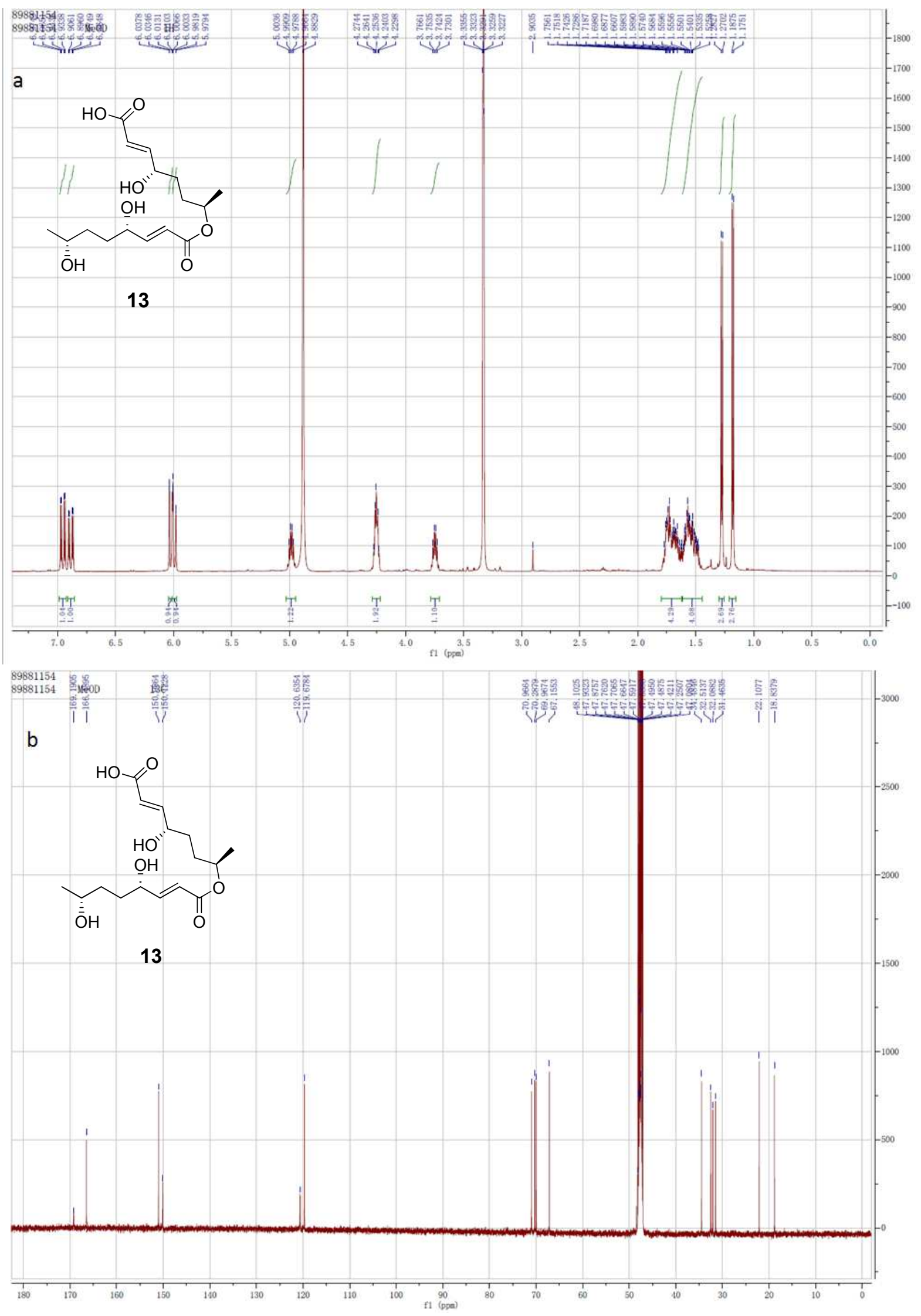

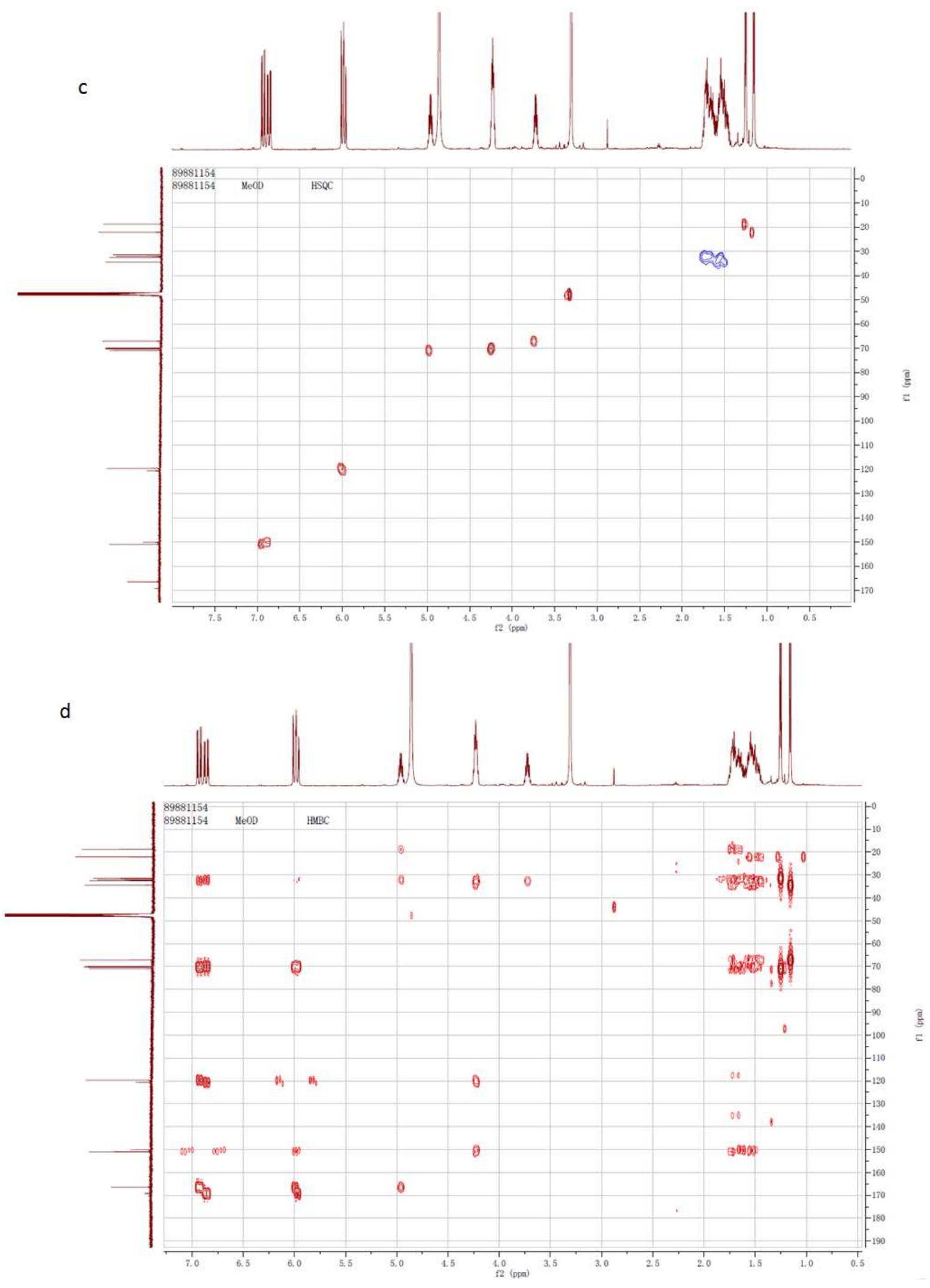


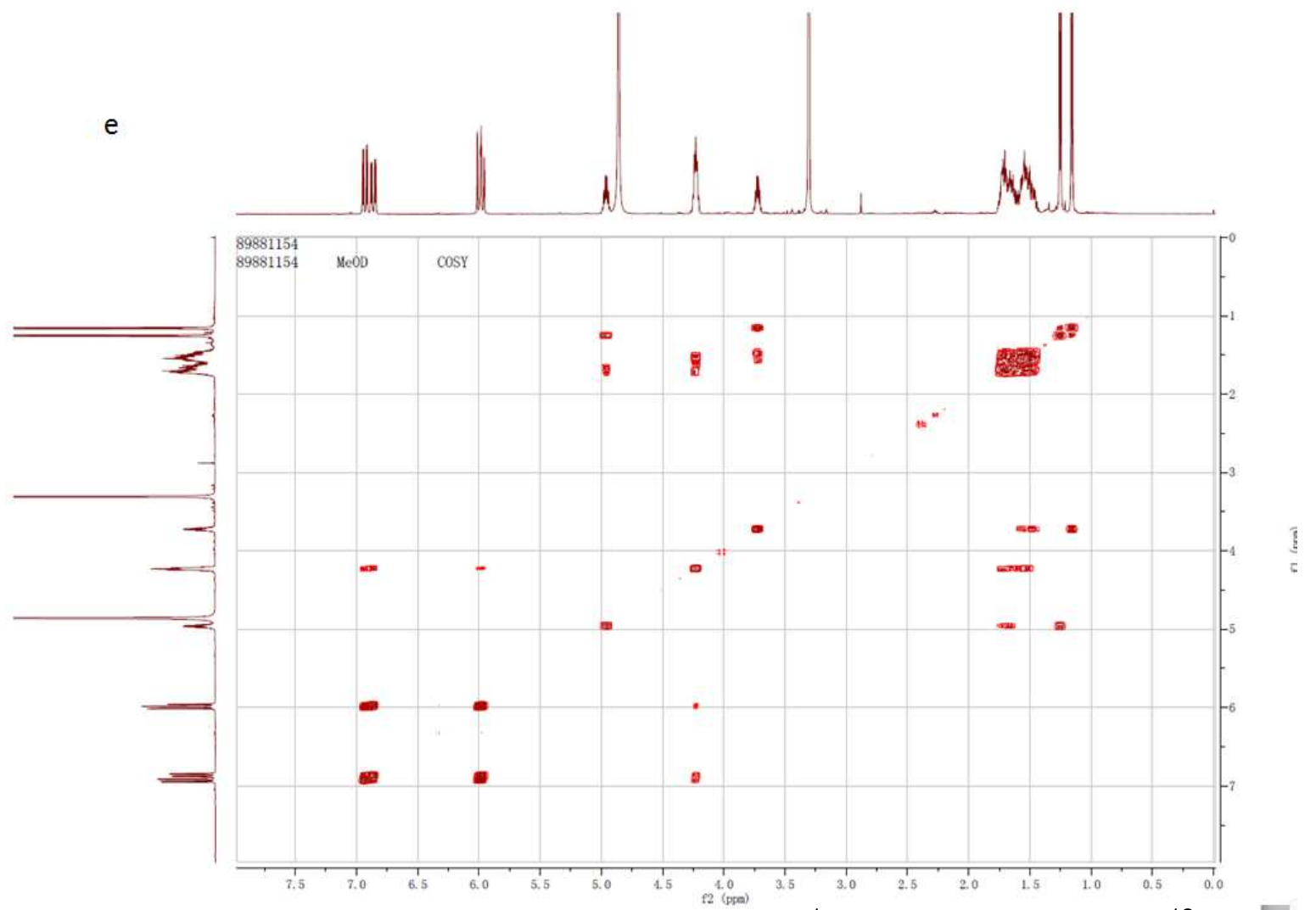

Figure S24. NMR analysis of 13 in methanol- $d_{4}$. (a) ${ }^{1} \mathrm{H}$ NMR spectrum. (b) ${ }^{13} \mathrm{C} N \bar{M} R$ spectrum. (c) HSQC spectrum. (d) HMBC spectrum. (e) ${ }^{1} \mathrm{H}-{ }^{1} \mathrm{H}$ COSY spectrum. 


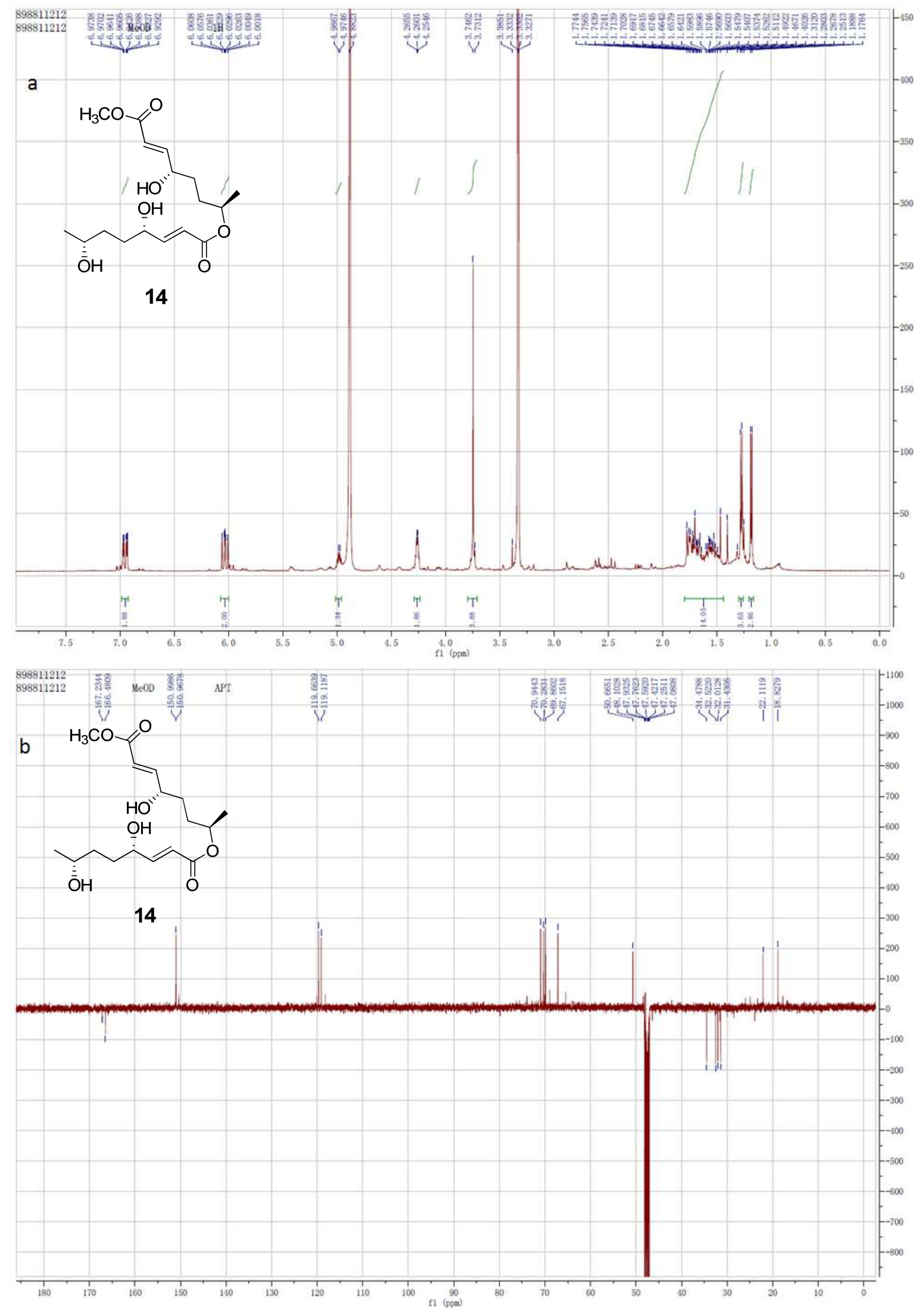




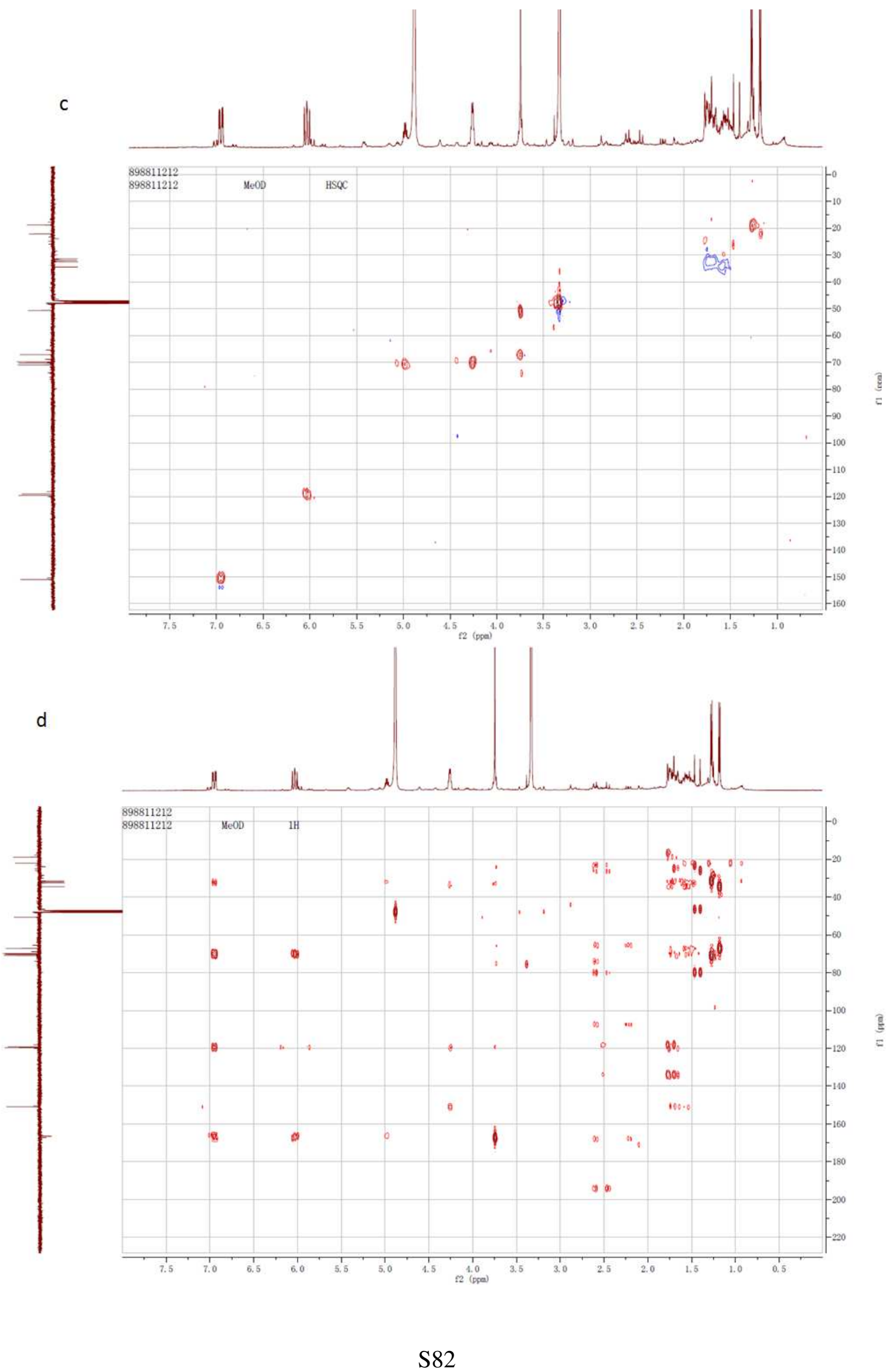




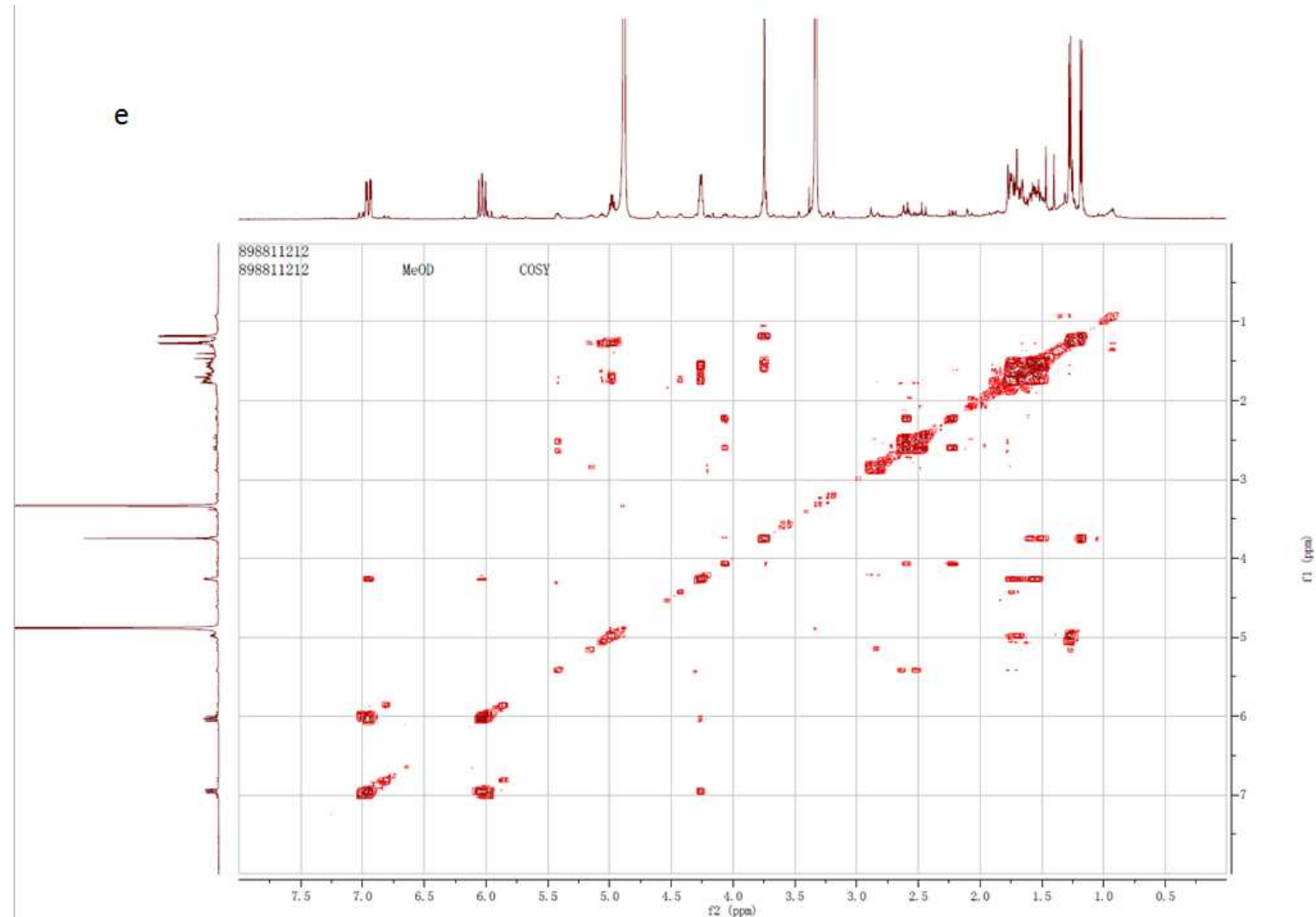

Figure S25. NMR analysis of 14 in methanol- $d_{4}$. (a) ${ }^{1} \mathrm{H}$ NMR spectrum. (b) ${ }^{13} \mathrm{C}$ NMR spectrum. (c) HSQC spectrum. (d) HMBC spectrum. (e) ${ }^{1} \mathrm{H}-{ }^{1} \mathrm{H}$ COSY spectrum. 

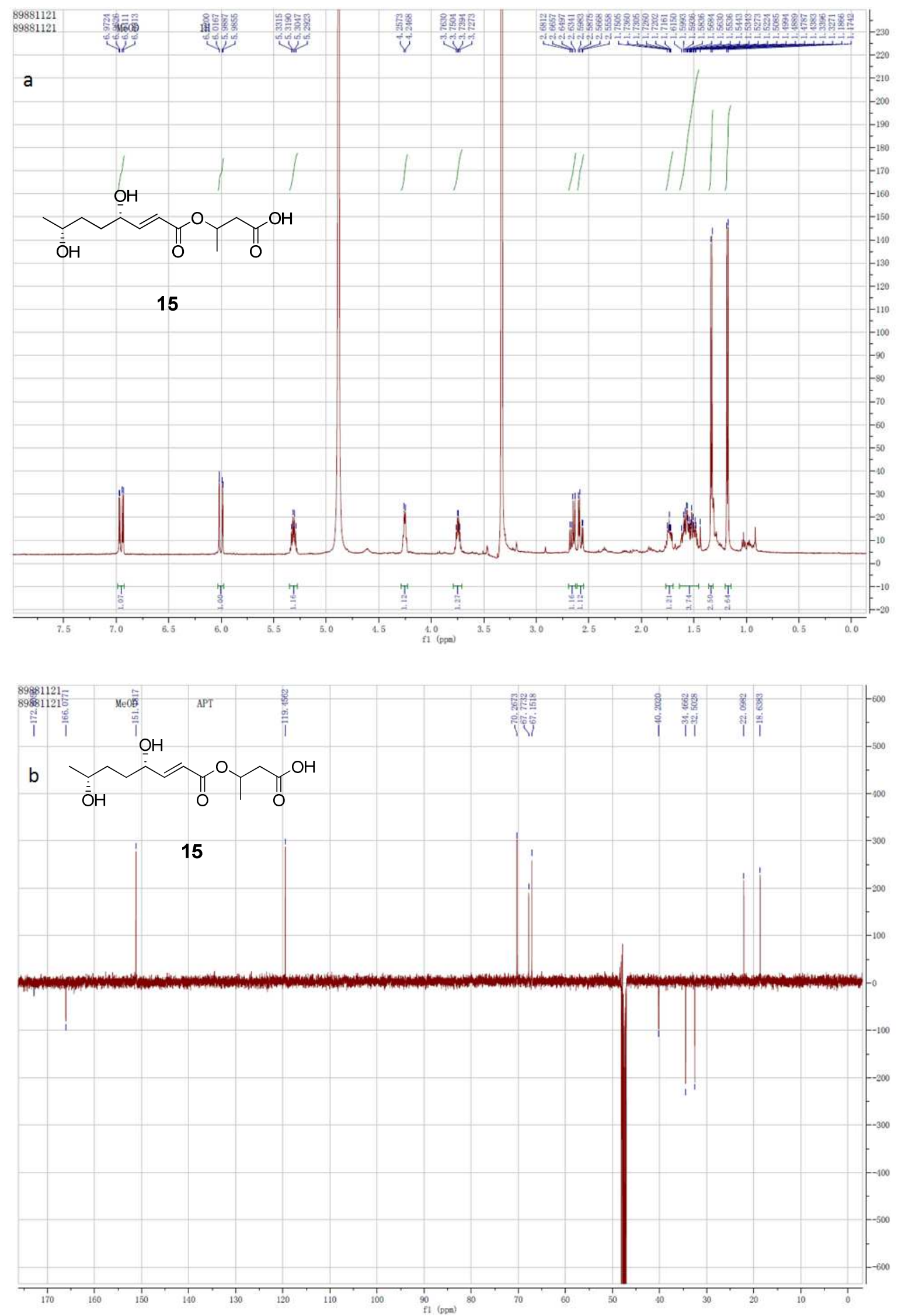


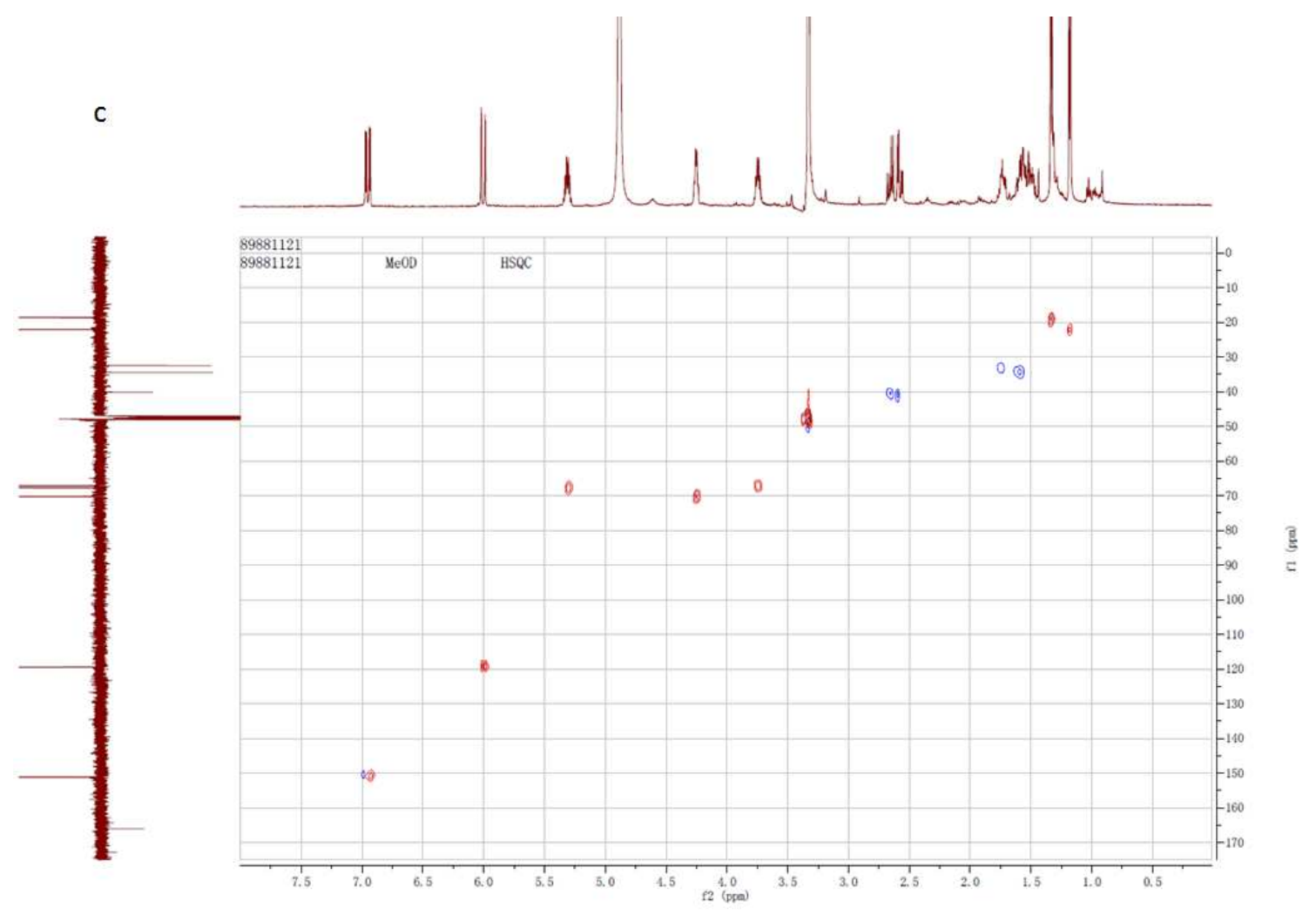

d

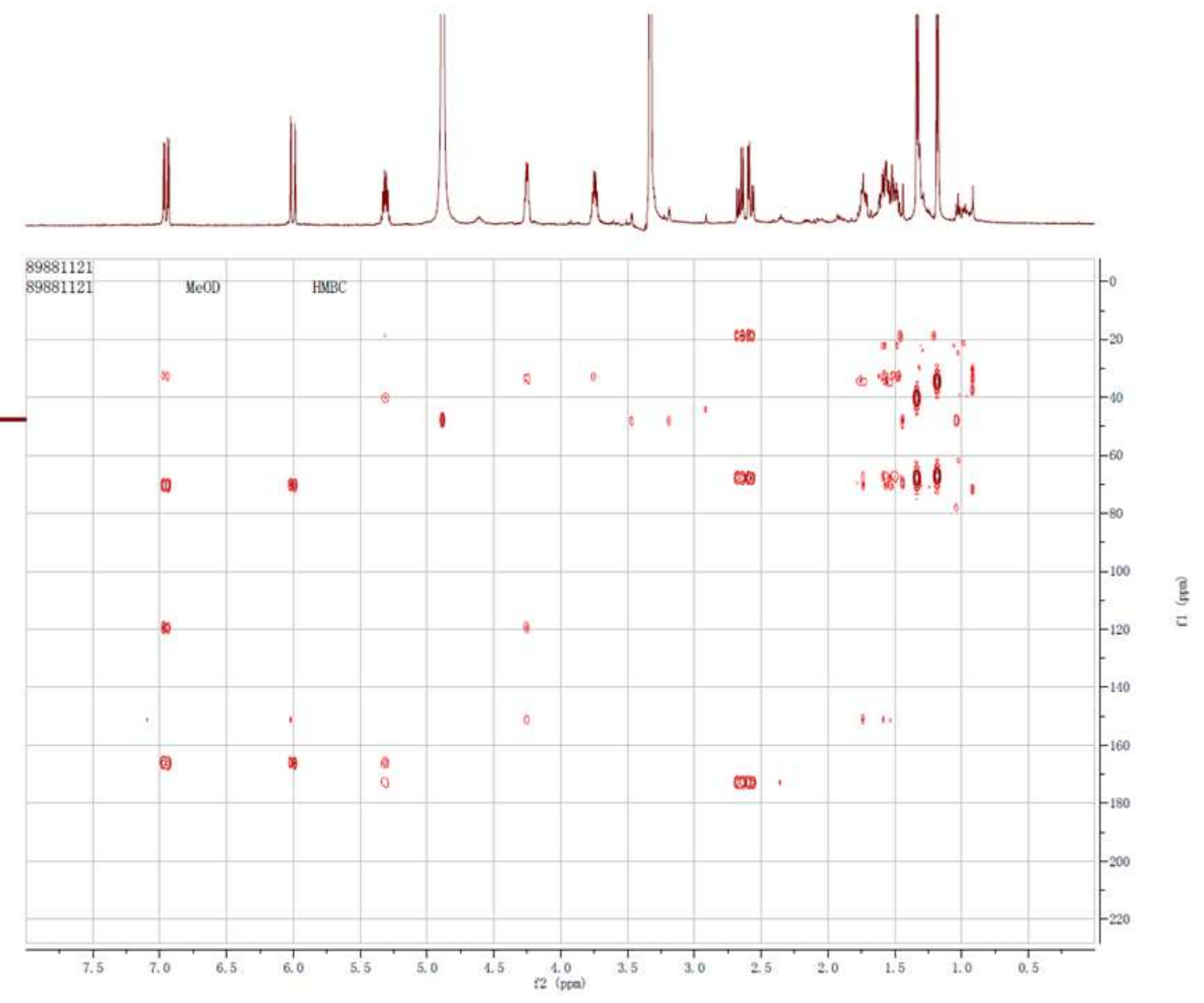

S85 


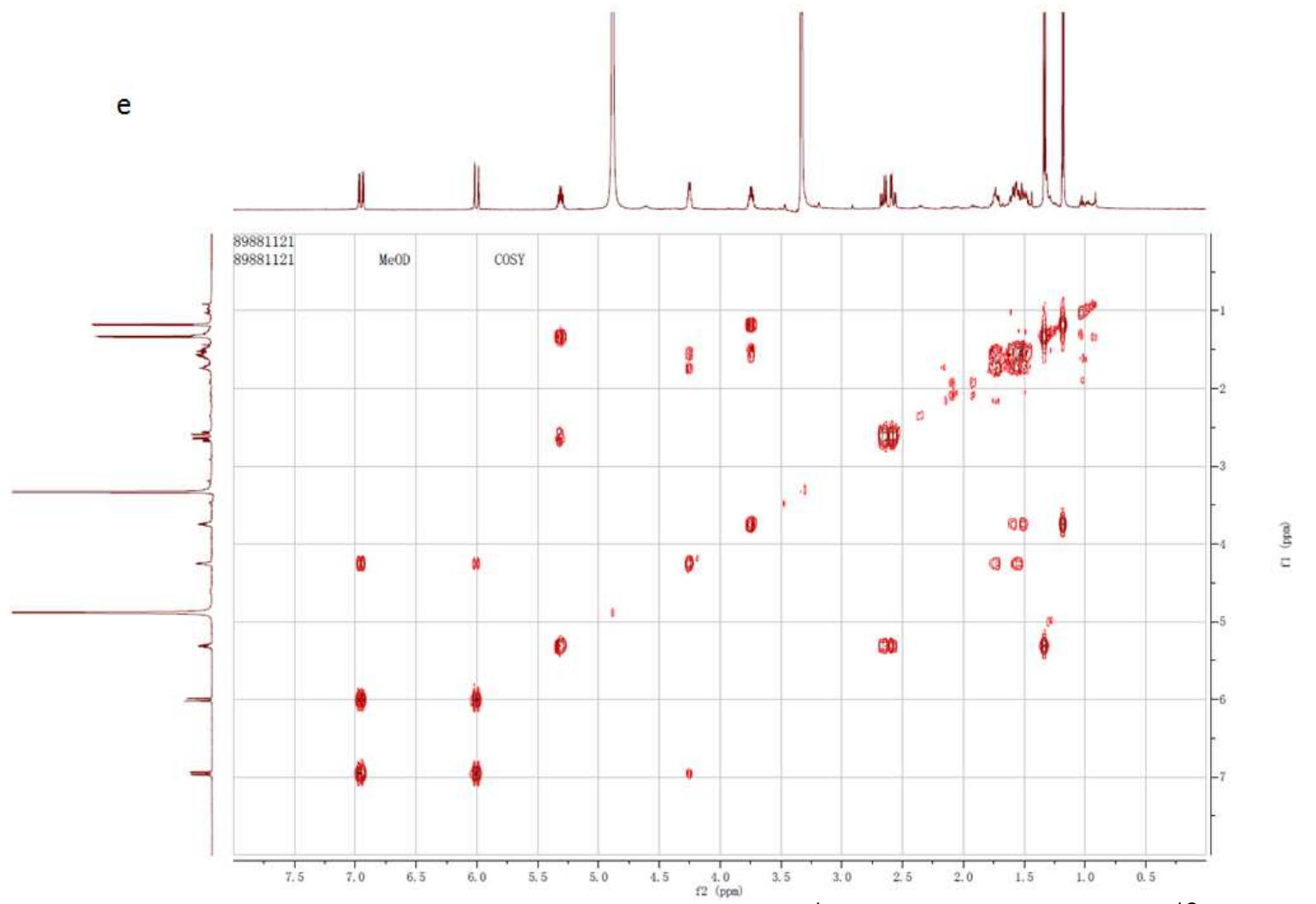

Figure S26. NMR analysis of 15 in methanol- $d_{4}$. (a) ${ }^{1} \mathrm{H}$ NMR spectrum. (b) ${ }^{13} \mathrm{C}$ NMR spectrum. (c) HSQC spectrum. (d) HMBC spectrum. (e) ${ }^{1} \mathrm{H}-{ }^{1} \mathrm{H}$ COSY spectrum. 

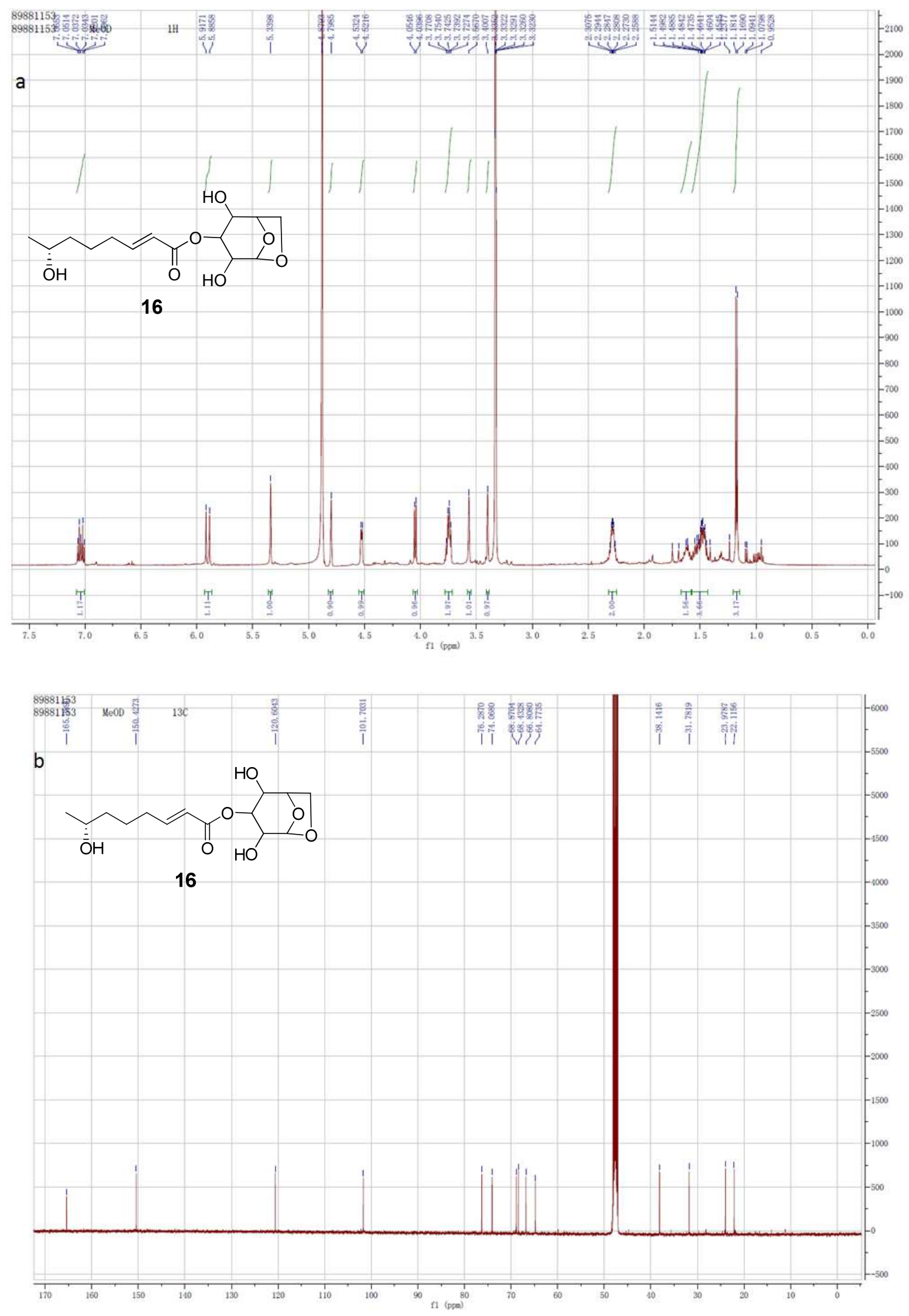

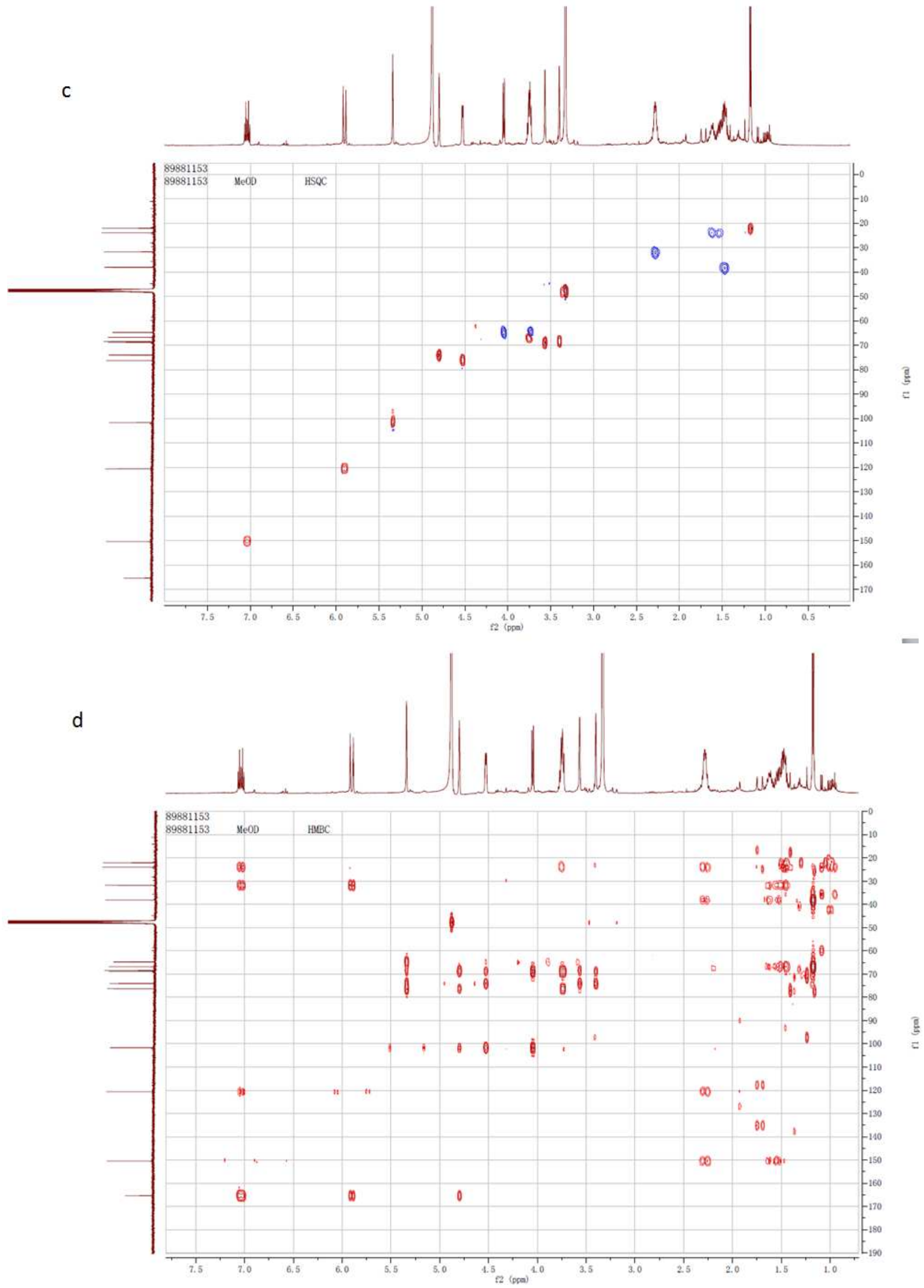


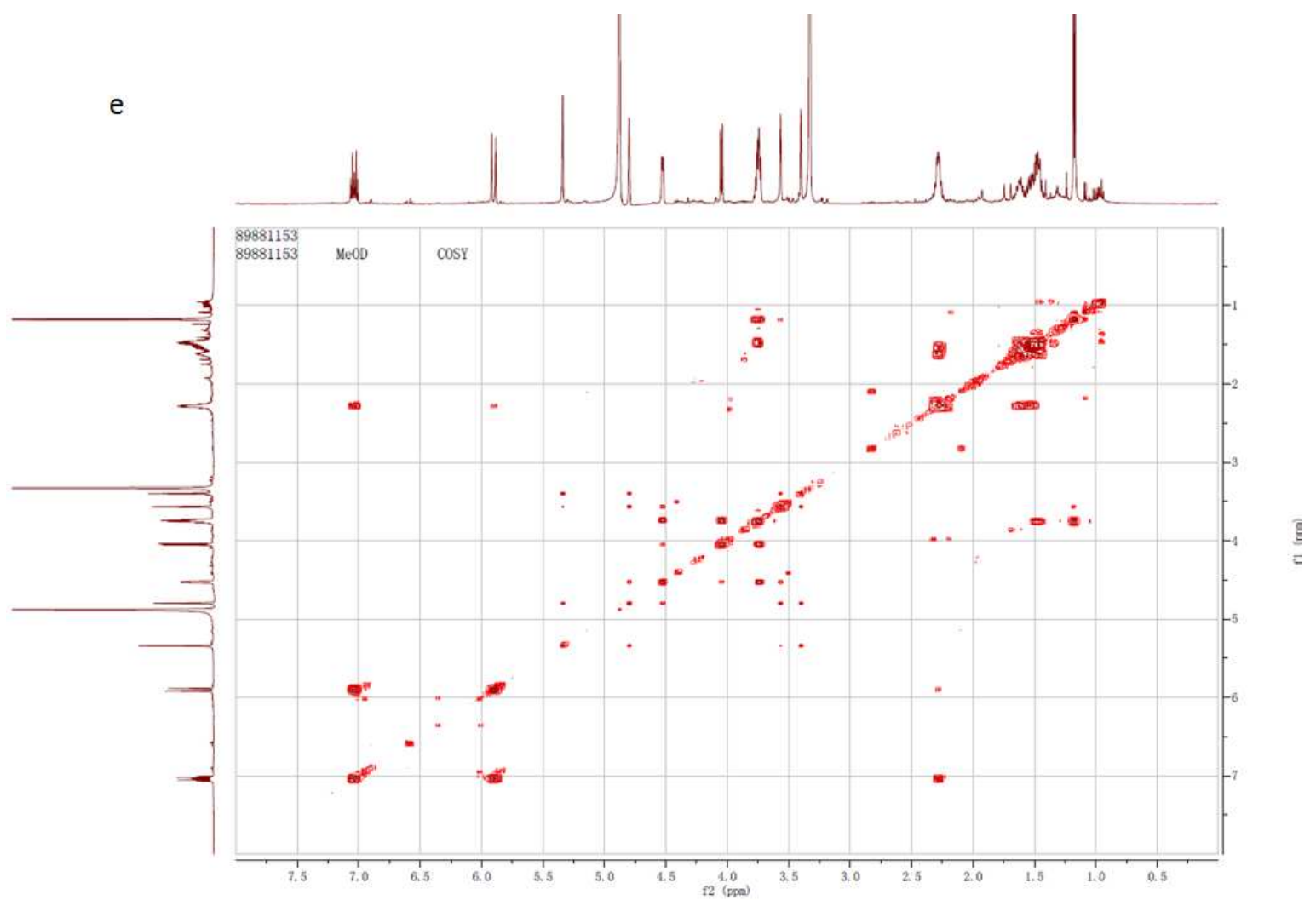

Figure S27. NMR analysis of 16 in methanol- $d_{4}$. (a) ${ }^{1} \mathrm{H}$ NMR spectrum. (b) ${ }^{13} \mathrm{C}$ NMR spectrum. (c) HSQC spectrum. (d) HMBC spectrum. (e) ${ }^{1} \mathrm{H}-{ }^{1} \mathrm{H}$ COSY spectrum. 

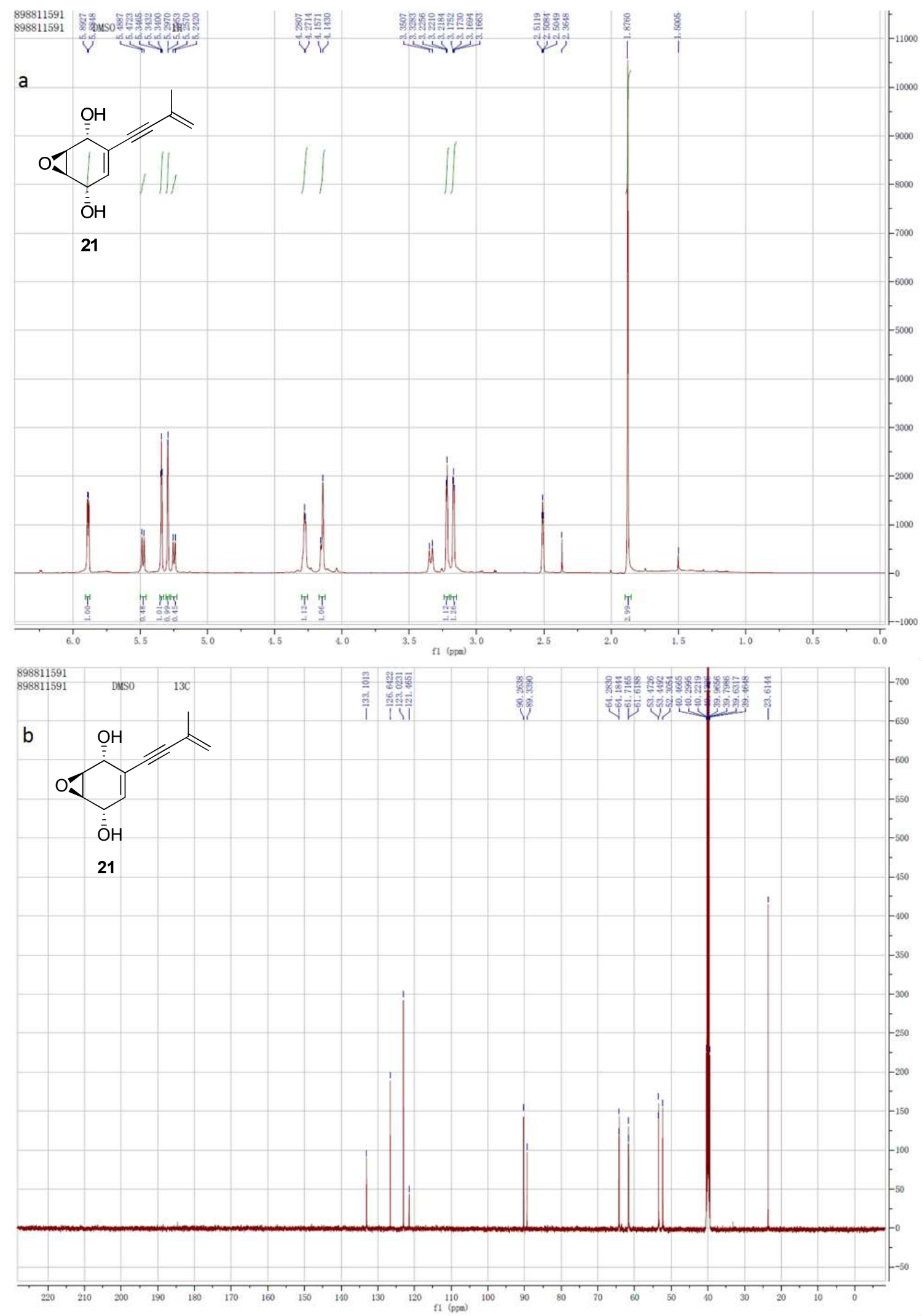

Figure S28. NMR analysis of 21 in DMSO- $d_{6}$. (a) ${ }^{1} \mathrm{H}$ NMR spectrum. (b) ${ }^{13} \mathrm{C}$ NMR spectrum. 


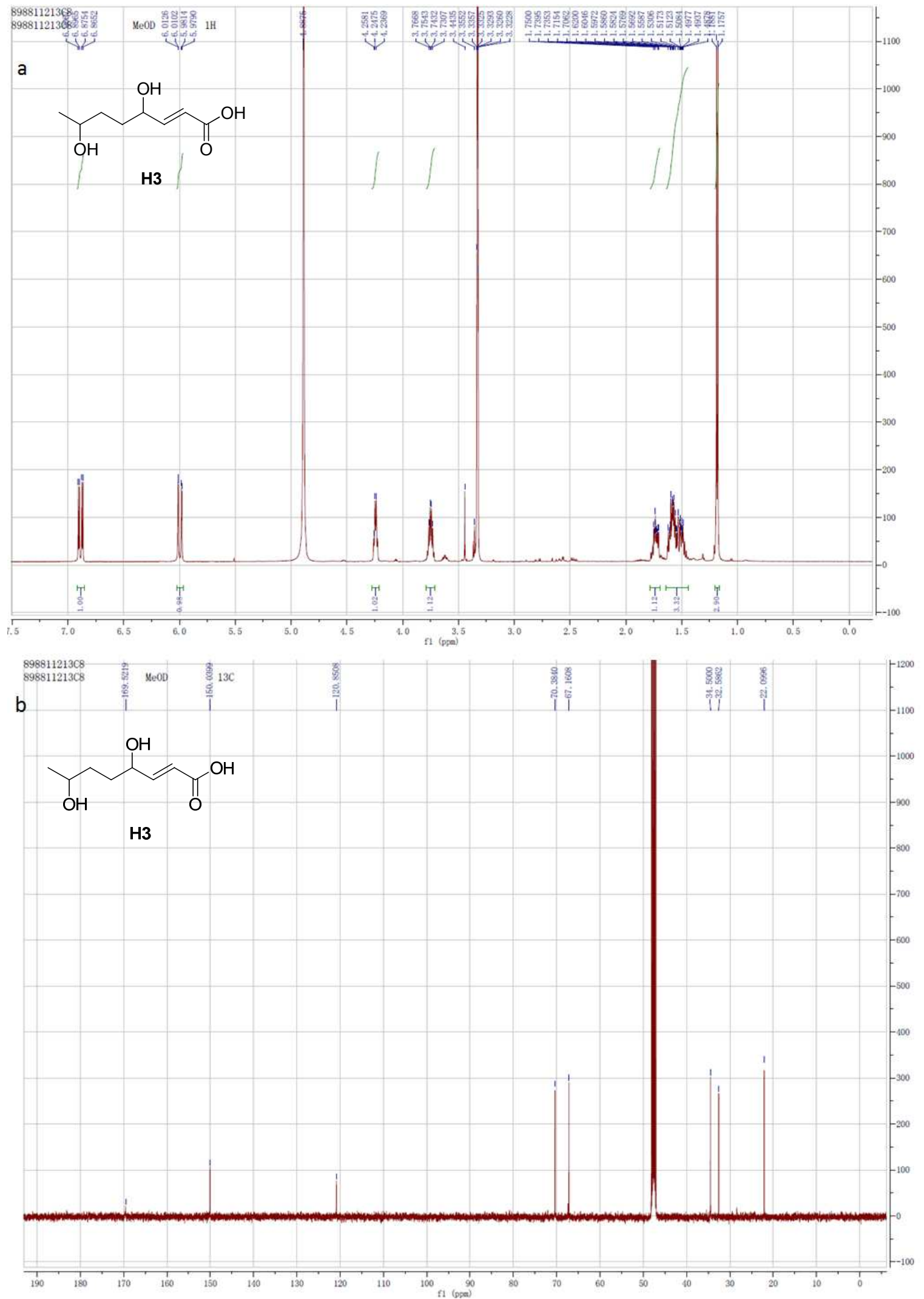

Figure S29. NMR analysis of $\mathbf{H 3}$ in methanol- $d_{4 .}$ (a) ${ }^{1} \mathrm{H}$ NMR spectrum. (b) ${ }^{13} \mathrm{C}$ NMR spectrum. 


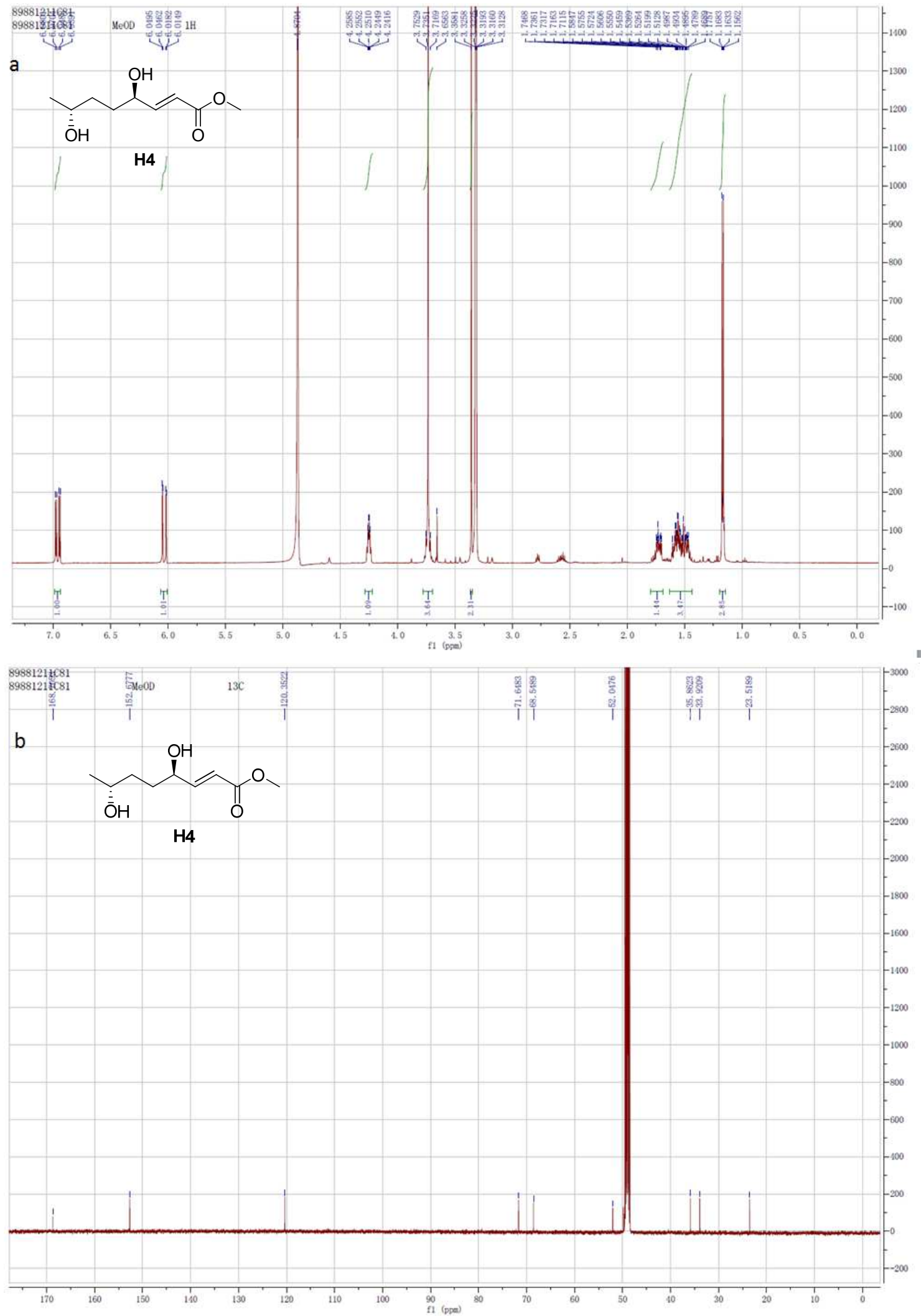

Figure S30. NMR analysis of $\mathbf{H} 4$ in methanol- $d_{4}$. (a) ${ }^{1} \mathrm{H}$ NMR spectrum. (b) ${ }^{13} \mathrm{C}$ NMR spectrum. 


\section{Supplementary References}

(1) Colot, H. V.; Park, G.; Turner, G. E.; Ringelberg, C.; Crew, C. M.; Litvinkova, L.; Weiss, R. L.; Borkovich, K. A.; Dunlap, J. C. Proc. Natl. Acad. Sci. USA 2006, 103, 10352-10357.

(2) Xu, X.; Liu, L.; Zhang, F.; Wang, W.; Li, J.; Guo, L.; Che, Y.; Liu, G. ChemBioChem 2014, 15, 284-292.

(3) Liu, L.; Liu, S. C.; Jiang, L. H.; Chen, X. L.; Guo, L. D.; Che, Y. S. Org. Lett. 2008, 10, 1397-1400.

(4) Wang, X.; Wu, F.; Liu, L.; Liu, X.; Che, Y.; Keller, N. P.; Guo, L.; Yin, W.-B. Fungal Genet. Biol. 2015. 81, 221-228.

(5) Dommerholt, F.; Thijs, L.; Zwanenburg, B. Tetrahedron Lett. 1991, 32, 1499-1502.

(6) Peng, J.; Gao, H.; Li, J.; Ai, J.; Geng, M.; Zhang, G.; Zhu, T.; Gu, Q.; Li, D. J. Org. Chem. 2014, 79, 7895-7904.

(7) Zhang, A.; Lu, P.; Dahl-Roshak, A. M.; Paress, P. S.; Kennedy, S.; Tkacz, J. S.; An, Z. Mol. Gen. Genomics. 2003, 268, 645-655.

(8) Yu, D.; Xu, F.; Zi, J.; Wang, S.; Gage, D.; Zeng, J.; Zhan, J. Metab. Eng. 2013, 18, 60-68. 